\title{
When Gold Meets Perfumes: Synthesis of Olfactive Compounds via Gold-Catalyzed Cycloisomerization Reactions
}

\author{
Romain Laher, ${ }^{\mathrm{a}}$ Christophe Marin ${ }^{\mathrm{b}}$ and Véronique Michelet ${ }^{\mathrm{a} *}$ \\ ${ }^{a}$ University Côte d'Azur, Institut de Chimie de Nice, UMR 7272 CNRS, Valrose Park, Faculty of Sciences, 06108, Nice Cedex 2, \\ France. \\ ${ }^{\mathrm{b}}$ Expressions Parfumées, 136 chemin de St Marc, 06130 Grasse, France.
}

\section{Supporting Information}

\section{General Remarks}

\section{Solvent purification:}

Ethers (tetrahydrofuran, diethylether) were distilled from sodium/benzophenone under argon atmosphere. Dichloromethane $\left(\mathrm{CH}_{2} \mathrm{Cl}_{2}\right)$, toluene and dimethylformamide (DMF) were distilled from calcium hydride under argon atmosphere. Solvents used in silica gel chromatographies were "analytical grade" and those used for extractions were "synthesis grade". Methanol, ethanol, allylic alcohol were used without further purification.

\section{Reagent purification:}

Reagents were purchased from Acros, Aldrich, Avocado and Fluorochem and used without purification unless otherwise stated.

\section{Chromatography:}

Thin-layer Chromatographies (T.L.C.) were carried out on ready-for-use silica plates silica gel 60 F254 art. 5554 (Merck). Products were eluted with mixture of solvents (volume/volume) and were visualized with U.V. light (254nm) and developed with $\mathrm{KMnO} 4$ (solution of potassium permanganate $(6 \mathrm{~g})$, potassium carbonate $(40 \mathrm{~g})$ and acetic acid $(0.5 \mathrm{~mL})$ in water $(600 \mathrm{~mL}))$ or Kagi (solution of p-anisaldehyde $(1.5 \mathrm{~mL})$, sulfuric acid $(1.5 \mathrm{~mL})$ and acetic acid $(0.3 \mathrm{~mL})$ in $27 \mathrm{~mL}$ of ethanol). Flash chromatography separations were made according to the method described by Still ${ }^{1}$ on silica gel $0,040-0,063 \mathrm{~mm}$ Art. 11567 (Merck) or Florisil gel 100-200 mesh (Aldrich). Gas Phase Chromatographies (G.C.) were carried out on a HP 5890 Serie II equipped with a $J \& W$ Scientific DB-1701 $(15 \mathrm{~m}, \varnothing=0.25 \mu \mathrm{m}, 50 \mathrm{kPa})$ capillary column using flame ionization detector.

\section{Analysis:}

Proton Nuclear Magnetic Resonance spectra $\left({ }^{1} \mathrm{H}\right.$ NMR) were recorded at $200 \mathrm{MHz}$ or $400 \mathrm{MHz}$ on a Brüker AC 200 or Avance 400 instrument. Chemical shifts $(\delta)$ were expressed in ppm with trimethylsilane (TMS) as external standard and the signal multiplicity was as follows :

$\mathrm{s}=$ singulet, $\mathrm{d}=$ doublet, $\mathrm{t}=$ triplet, $\mathrm{q}=$ quadruplet, $\mathrm{m}=$ multiplet, $\mathrm{br} .=$ broad.

Carbon Nuclear Magnetic Resonance spectra $\left({ }^{13} \mathrm{C}\right.$ NMR) were recorded at $50 \mathrm{MHz}$ or $100 \mathrm{MHz}$ on a Brüker AC 200 or Avance 400 instrument. Chemical shifts $(\delta)$ were expressed in ppm with trimethylsilane (TMS) as external standard. NMR analysis were all carried out in $\mathrm{CDCl}_{3}$ as deuterated solvent. Mass spectra were accomplished by the ICN Mass Spectroscopy Service, on a Hewlett<Packard HP 5989 A spectrometer via either direct introduction or GC/MS coupling with a Hewlett Packard HP 5890 class II fit with a Chrompack CPSi $15 \mathrm{CB}$ capillary column. Ionization was obtained either by electronic impact (EI) chemical ionization with ammonia (DCI/NH3). High resolution Mass Spectroscopy (HRMS) analyses were performed at the Institut de Chimie de Nice in ultra-high performance liquid chromatography coupled with hybrid quadrupole-orbitrap mass spectrometer (UPLC-Q-Exactive/MS).

\footnotetext{
${ }^{1}$ Still, W. C.; Khan, M.; Mitra, A. J. Org. Chem. 1978, 43, 2923.
} 
Content

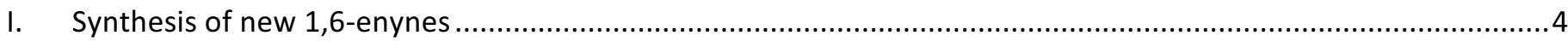

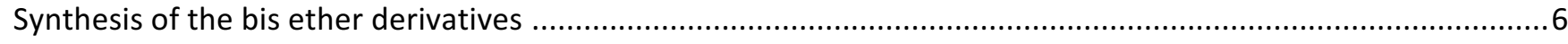

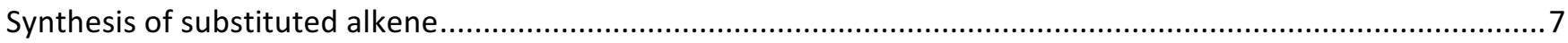

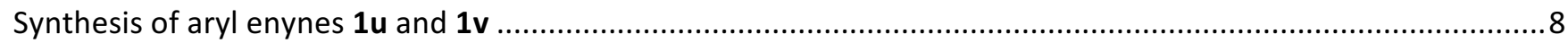

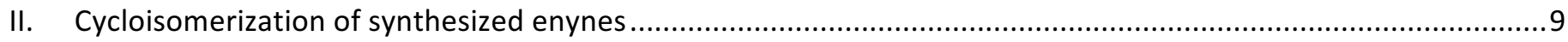

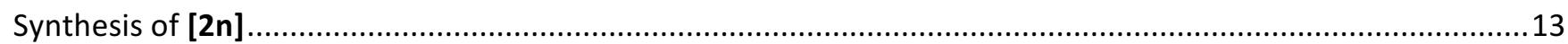

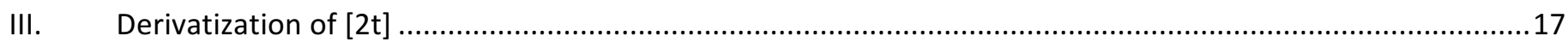

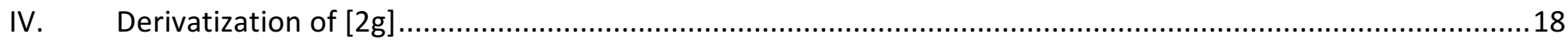

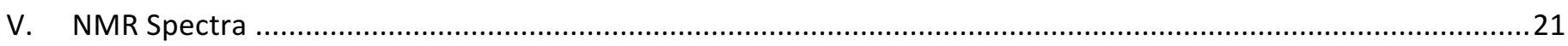

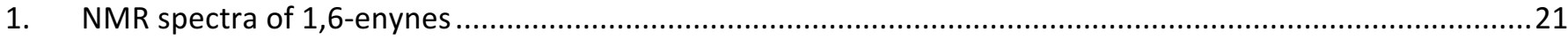

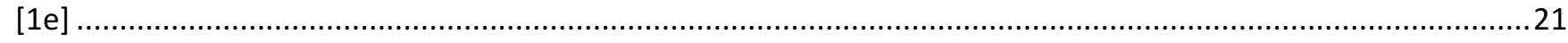

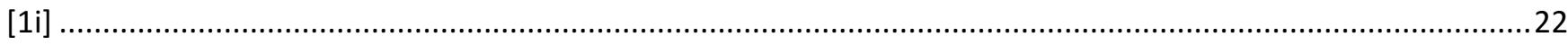

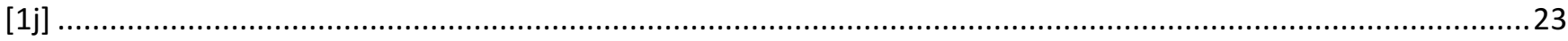

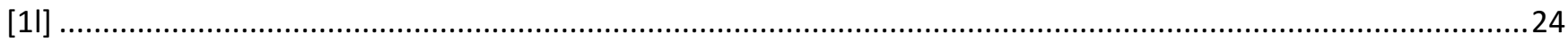

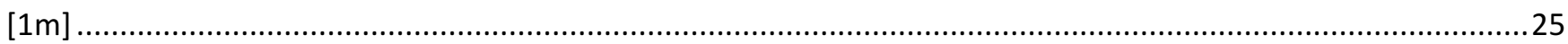

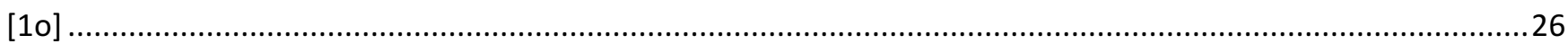

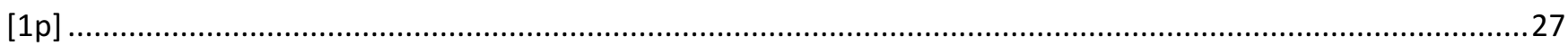

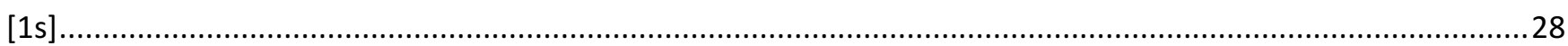

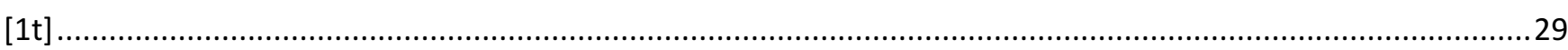

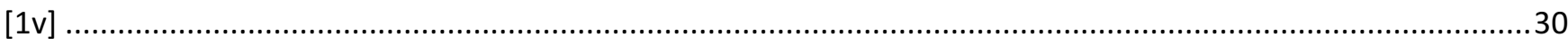

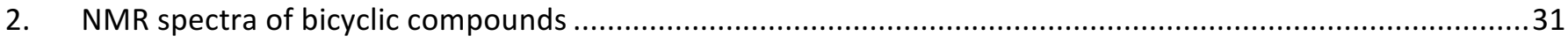

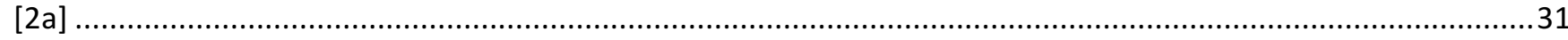

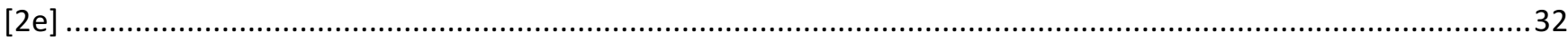

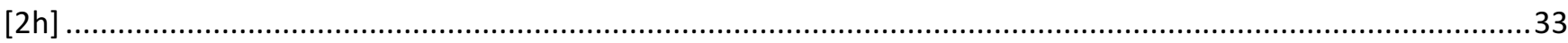

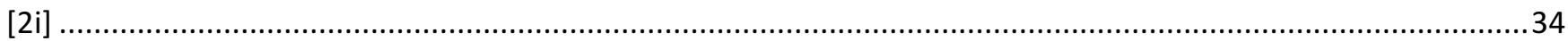

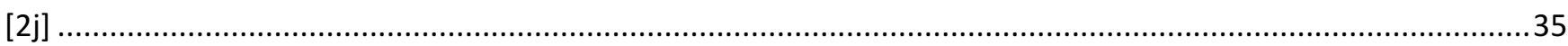

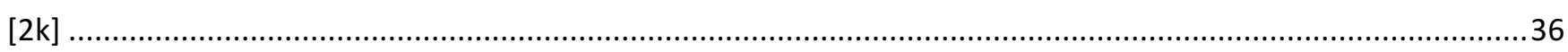

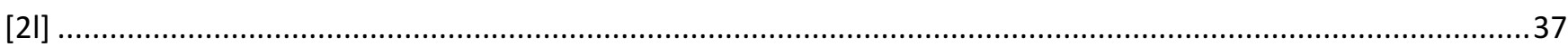

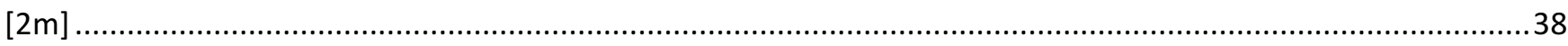

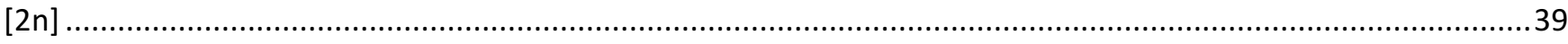

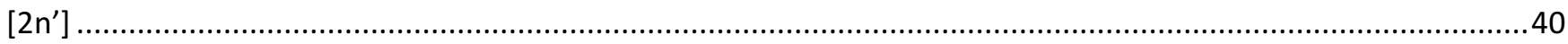

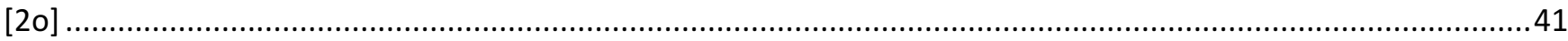

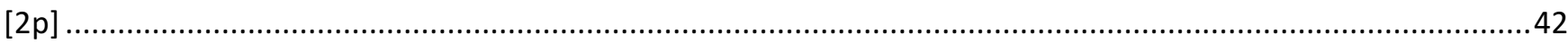

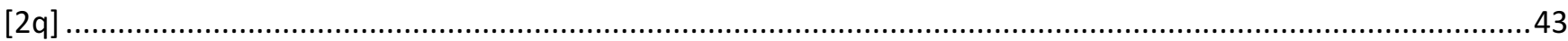

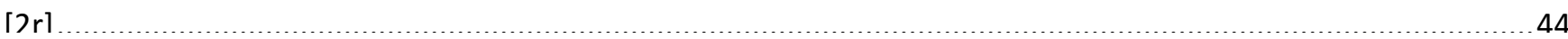


[v]

[4]. 49

[5] 50

[6] .52

[7] .53

[8a] .54

[8b] 55<smiles>[R]C#CCO</smiles><smiles>[R]C#CCOCC([R])=C([R])[R]</smiles>

1a-v

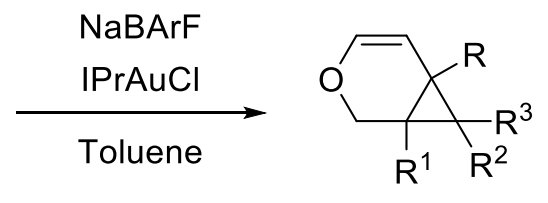

2a-v

\section{General Procedure (1): Synthesis of oxygenated enynes :}

To a suspension of $\mathbf{N a H}(1.1 \mathrm{eq}, \mathrm{M}=24)$ in freshly distilled DMF $(0.67 \mathrm{M})$ at $0^{\circ} \mathrm{C}$ was added the alcohol ( 1 eq) dropwise. The reaction was allowed to warm up to $\mathrm{rt}$ and stirred for 30 minutes. Alkene bromide (1.5 eq) is then added portionwise at $\mathrm{rt}$. The reaction mixture is stirred until completion monitored by TLC.

Reaction is quenched with $15 \mathrm{~mL}$ of an aqueous solution of $\mathrm{HCl}(3.5 \%)$. Aqueous phase is extracted thrice with $\mathrm{Et}_{2} \mathrm{O}(15 \mathrm{~mL})$. Combined organic phase are dried over $\mathrm{MgSO}_{4}$, filtrated and concentrated in vacuuo. The crude product was purified by silica gel flash chromatography if necessary (petroleum ether/ $\mathrm{Et}_{2} \mathrm{O}, 90 / 10$ ).

\section{General Procedure (2): Achiral Au-catalyzed cycloisomerization of enynes :}

To a suspension of IPrAuCl ( $2 \mathrm{~mol} \%, \mathrm{M}=621.01)$ and $\mathbf{N a B A r}{ }^{\mathbf{F}}(2 \mathrm{~mol} \%, \mathrm{M}=886.20)$ in distilled Toluene (1M) was added the enyne in distilled Toluene $(1 \mathrm{M})$. The reaction was stirred at $50^{\circ} \mathrm{C}$ using an oil bath until complete conversion of the enyne monitored by TLC. After completion of the reaction, the mixture was filtered through a short pad of silica gel (25\%), aluminum oxide $(75 \%)$ and a large amount of celite or through a pad of Florisil@. (cyclohexane/EtOAc, 50/50) and the solvent were evaporated under reduced pressure. The crude product was purified by silica gel flash chromatography if necessary (petroleum ether/ $\left.\mathrm{Et}_{2} \mathrm{O}, 95 / 5\right)$. 


\title{
I. Synthesis of new 1,6-enynes
}

Enynes $1 \mathbf{a},{ }^{2} \mathbf{1 b},{ }^{3} \mathbf{1 c},{ }^{4} \mathbf{1 d},{ }^{5} \mathbf{1 f},{ }^{6} \mathbf{1 g},{ }^{7} \mathbf{1} \mathbf{k},{ }^{8} \mathbf{1 n},{ }^{9} \mathbf{1 n},{ }^{10} \mathbf{1 q}{ }^{11} \mathbf{1 r},{ }^{12} \mathbf{1 u},{ }^{13}$ as well as intermediates such as $\mathbf{1 p}$-CHO, ${ }^{14} \mathbf{1 p}$ '$\mathbf{O H},{ }^{15} \mathbf{1 q},{ }^{16} \mathbf{1 r},{ }^{17} \mathbf{1} \mathbf{s},{ }^{17} \mathbf{1 t},{ }^{18} \mathbf{1},{ }^{19}$ were prepared according to known procedures and spectra are in accordance to published results.

1-(allyloxy)dec-2-yne [1e]

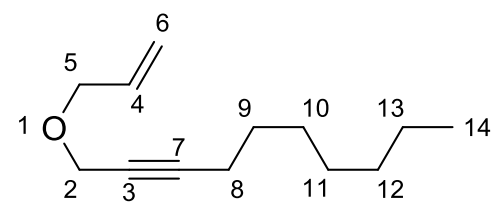

\author{
Formula : $\mathrm{C}_{13} \mathrm{H}_{22} \mathrm{O}$ \\ $\mathrm{M}: 194.32{\mathrm{~g} . \mathrm{mol}^{-1}}^{-1}$ \\ Aspect : Colorless oil \\ $\mathrm{R}_{\mathrm{f}}=0.77$ (petroleum ether $/ \mathrm{Et}_{2} \mathrm{O}, 90 / 10 ; \mathrm{KMnO}_{4}$ )
}

According to the experimental procedure (1) and starting from $940 \mu \mathrm{L}$ ( 5 mmol, 1 eq., $\mathrm{M}=154.25$, $\mathrm{d}=0.85)$ of 2-decyn-1-ol, 650 $\mu \mathrm{L}(7.5 \mathrm{mmol}, 1.5 \mathrm{eq}, \mathrm{M}=120.98, \mathrm{~d}=1.40)$ of allyl bromide and $144 \mathrm{mg}(9 \mathrm{mmol}, 1.2 \mathrm{eq}, \mathrm{M}=24)$ of sodium hydride in DMF $(0.67 \mathrm{M})$, the 1-(allyloxy)dec-2-yne [1e] was obtained as an Colorless oil $(1.15 \mathrm{~g}, 79$ \%).

${ }^{1} \mathrm{H}$ NMR $\left(\mathrm{CDCl}_{3}, 400 \mathrm{MHz} ; \delta\right.$ ppm) :

$5.92(\mathrm{ddt}, J=17.3,10.4,5.8 \mathrm{~Hz}, 1 \mathrm{H}, 4), 5.30\left(\mathrm{dq}, J=17.2,1.6 \mathrm{~Hz}, 1 \mathrm{H}, \mathbf{6}^{\prime \prime}\right), 5.21\left(\mathrm{dq}, J=10.4,1.3 \mathrm{~Hz}, 1 \mathrm{H}, \mathbf{6}^{\prime}\right), 4.14(\mathrm{t}, J=2.2$ $\mathrm{Hz}, 2 \mathrm{H}, 2), 4.05(\mathrm{dt}, J=5.8,1.4 \mathrm{~Hz}, 2 \mathrm{H}, \mathbf{5}), 2.22(\mathrm{tt}, J=7.1,2.2 \mathrm{~Hz}, 2 \mathrm{H}, \mathbf{8}), 1.57-1.45$ (m, 2H, 9), $1.43-1.34$ (m, $2 \mathrm{H}, \mathbf{1 0}), 1.29$ (pd, $J=7.5,4.7 \mathrm{~Hz}, 6 \mathrm{H}, 11,12,13), 0.92-0.84(\mathrm{~m}, 2 \mathrm{H}, 14)$.

${ }^{13} \mathrm{C}$ NMR $\left(\mathrm{CDCl}_{3}, 101 \mathrm{MHz} ; \delta\right.$ ppm) :

134.24 (CH, 4), $117.60\left(\mathrm{CH}_{2}, \mathbf{6}\right), 87.16(\mathrm{C}, 7), 75.76(\mathrm{C}, \mathbf{3}), 70.40\left(\mathrm{CH}_{2}, \mathbf{5}\right), 57.75\left(\mathrm{CH}_{2}, 2\right), 31.72\left(\mathrm{CH}_{2}, \mathbf{1 2}\right), 28.82\left(\mathrm{CH}_{2}, \mathbf{1 1}\right)$, $28.78\left(\mathrm{CH}_{2}, \mathbf{1 0}\right), 28.61\left(\mathrm{CH}_{2}, 9\right), 22.61\left(\mathrm{CH}_{2}, 13\right), 18.76\left(\mathrm{CH}_{2}, \mathbf{8}\right), 14.06\left(\mathrm{CH}_{3}, 14\right)$.

HRMS (EI) m/z : $[\mathrm{M}+\mathrm{H}]^{+}$Calcd for $\mathrm{C}_{13} \mathrm{H}_{22} \mathrm{O}$ 195.1749; Found 195.1742

1-((2-methylallyl)oxy)pent-2-yne [1/h]

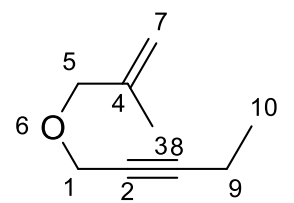

\author{
Formula : $\mathrm{C}_{9} \mathrm{H}_{14} \mathrm{O}$ \\ $\mathrm{M}: 138.21{\mathrm{~g} \cdot \mathrm{mol}^{-1}}^{-1}$ \\ Aspect : Colorless oil \\ $\mathrm{R}_{\mathrm{f}}=0.65$ (petroleum ether $/ \mathrm{Et}_{2} \mathrm{O}, 96 / 4 ; \mathrm{KMnO}_{4}$ ) \\ $\mathrm{GC}: 4.295 \mathrm{~min}$
}

\footnotetext{
${ }^{2}$ Nieto-Oberhuber, C.; Pérez-Galán, P.; Herrero-Gómez, E.; Lauterbach, T.; Rodríguez, C.; López, S.; Bour, C.; Rosellón, A.; Cárdenas, D. J.; Echavarren, A. M. J. Am. Chem. Soc. 2008, 130 (1), 269-279.

${ }^{3}$ Teller, H.; Corbet, M.; Mantilli, L.; Gopakumar, G.; Goddard, R.; Thiel, W.; Fürstner, A. J. Am. Chem. Soc. 2012, 134 (37), 15331-15342.

${ }^{4}$ Blum, J.; Beer-Kraft, H.; Badrieh, Y. J. Org. Chem. 1995, 60 (17), 5567-5569.

${ }^{5}$ Conde, N.; SanMartin, R.; Herrero, M. T.; Domínguez, E. Adv. Synth. Catal. 2016, 358 (20), 3283-3292.

${ }^{6}$ Cabrera-Lobera, N.; Rodríguez-Salamanca, P.; Nieto-Carmona, J. C.; Buñuel, E.; Cárdenas, D. J. Chem. Eur. J. 2018, 24(4), 784-788; Lin, A.; Zhang, Z.-W.; Yang, J. Org. Lett. 2014, 16 (2), 386-389; Xie, Y.; Yu, M.; Zhang, Y. Synthesis 2011, 2011 (17), 2803-2809; Park, K. H.; Chung, Y. K. Advanced Synthesis \& Catalysis 2005, 347 (6), 854-866.

${ }^{7}$ He, Y.-T.; Wang, Q.; Zhao, J.; Wang, X.-Z.; Qiu, Y.-F.; Yang, Y.-C.; Hu, J.-Y.; Liu, X.-Y.; Liang, Y.-M. Advanced Synthesis \& Catalysis 2015, 357 (14-15), 3069-3075. Wang, Y.-Q.; He, Y.-T.; Zhang, L.-L.; Wu, X.-X.; Liu, X.-Y.; Liang, Y.-M. Org. Lett. 2015, 17 (17), 42804283. He, Y.-T.; Li, L.-H.; Zhou, Z.-Z.; Hua, H.-L.; Qiu, Y.-F.; Liu, X.-Y.; Liang, Y.-M. Org. Lett. 2014, 16 (15), $3896-3899$.

${ }^{8}$ Journet, M.; Malacria, M. J. Org. Chem. 1992, 57 (11), 3085-3093.

Dzik, W. I.; Xu, X.; Zhang, X. P.; Reek, J. N. H.; de Bruin, B. J. Am. Chem. Soc. 2010, 132 (31), 10891-10902.

${ }^{10}$ Clavier, H.; Correa, A.; Escudero-Adán, E. C.; Benet-Buchholz, J.; Cavallo, L.; Nolan, S. P. Chem. Eur. J. 2009, 15 (39), $10244-10254$.

${ }^{11}$ Wang, Y.-Q.; He, Y.-T.; Zhang, L.-L.; Wu, X.-X.; Liu, X.-Y.; Liang, Y.-M. Org. Lett. 2015, 17 (17), 4280-4283.

12 Diao, Y.; Zuo, Z.; Wang, H.; Liu, J.; Luan, X. Org. Biomol. Chem. 2017, 15 (21), 4601-4608.

${ }^{13}$ Gao, P.; Yan, X.-B.; Tao, T.; Yang, F.; He, T.; Song, X.-R.; Liu, X.-Y.; Liang, Y.-M. Chem. Eur. J. 2013, 19 (43), $14420-14424$.

${ }^{14}$ Erkkilä, A.; Pihko, P. M., Eur. J. Org. Chem., 2007, 4205.

${ }^{15}$ Faulkner, A.; Scott, J. S.; Bower, J. F. J. Am. Chem. Soc. 2015, 137 (22), 7224-7230.

${ }^{16}$ Chi, X.; Meng, L.; Pang, Q.; Guo, L.; Liu, Q.; Zhao, P.; Zhang, D.; Sun, F.; Li, X.; Liu, H. Asian J. Org. Chem. 2019, 8 (12), $2201-2204$.

${ }^{17}$ Chen, Z.; Liang, P.; Xu, F.; Deng, Z.; Long, L.; Luo, G.; Ye, M. J. Org. Chem. 2019, 84 (19), 12639-12647.

${ }^{18}$ Ignatiuk, Ż. A.; Janicki, M. J.; Góra, R. W.; Konieczny, K.; Kowalczyk, R. Adv. Synth. Catal. 2019, 361 (5), $1108-1116$.

${ }^{19}$ Xu, Y.; Hong, Y. J.; Tantillo, D. J.; Brown, M. K. Org. Lett. 2017, 19 (14), 3703-3706.
} 
According to the experimental procedure (1) and starting from $1.4 \mathrm{~mL}(15 \mathrm{mmol}, 1$ eq., $\mathrm{M}=84.12, \mathrm{~d}=1.41)$ of 2-pentyn-1-ol, 2.3 $\mathrm{mL}$ (22.5 mmol, $1.5 \mathrm{eq}, \mathrm{M}=135, \mathrm{~d}=1.34)$ of 1-bromo-2-methyl-prop-2-ene and $432 \mathrm{mg}(18 \mathrm{mmol}, 1.2 \mathrm{eq}, \mathrm{M}=24)$ of sodium hydride in DMF (0.67 M), the 1-((2-methylallyl)oxy)pent-2-yne [1h] was obtained as an Colorless oil (664 mg, $32 \%)$.

${ }^{1} \mathrm{H}$ NMR $\left(\mathrm{CDCl}_{3}, 200 \mathrm{MHz} ; \delta\right.$ ppm):

$5.04-4.88(\mathrm{~m}, 2 \mathrm{H}, 7), 4.10(\mathrm{t}, J=2.2 \mathrm{~Hz}, 2 \mathrm{H}, \mathbf{1}), 4.00-3.85(\mathrm{~m}, 2 \mathrm{H}, \mathbf{5}), 2.24(\mathrm{qt}, J=7.4,2.2 \mathrm{~Hz}, 2 \mathrm{H}, \mathbf{9}), 1.87-1.67(\mathrm{~m}, 3 \mathrm{H}, \mathbf{3})$, $1.15(\mathrm{t}, J=7.5 \mathrm{~Hz}, 3 \mathrm{H}, \mathbf{1 0})$.

${ }^{13} \mathrm{C}$ NMR $\left(\mathrm{CDCl}_{3}, 50 \mathrm{MHz} ; \delta\right.$ ppm): $141.70(\mathrm{C}, \mathbf{4}), 112.73\left(\mathrm{CH}_{2}, 7\right), 88.23(\mathrm{C}, \mathbf{2}), 75.28(\mathrm{C}, \mathbf{8}), 73.47(\mathrm{C}, \mathbf{5}), 57.57\left(\mathrm{CH}_{2}, \mathbf{1}\right), 19.55\left(\mathrm{CH}_{3}, \mathbf{3}\right), 13.81\left(\mathrm{CH}_{3}, \mathbf{1 0}\right), 12.46$ $\left(\mathrm{CH}_{2}, 9\right)$.

HRMS (EI) m/z: $[\mathrm{M}+\mathrm{H}]^{+}$Calcd for $\mathrm{C}_{9} \mathrm{H}_{14} \mathrm{O}$ 139.1123; Found: 139.1118

1-((2-methylallyl)oxy)hex-2-yne [1i]

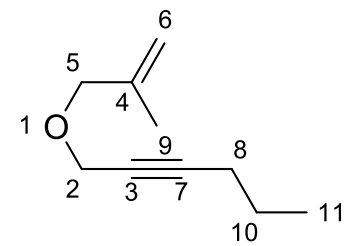

\author{
Formula : $\mathrm{C}_{10} \mathrm{H}_{16} \mathrm{O}$ \\ $\mathrm{M}: 152.24 \mathrm{~g} \cdot \mathrm{mol}^{-1}$ \\ Aspect : Light yellow oil \\ $\mathrm{R}_{\mathrm{f}}=0.68$ (petroleum ether/ $\mathrm{Et}_{2} \mathrm{O}, 96 / 4 ; \mathrm{KMnO}_{4}$ ) \\ $\mathrm{GC}: 5.36 \mathrm{~min}$
}

According to the experimental procedure (1) and starting from $1.7 \mathrm{~mL}(15 \mathrm{mmol}, 1$ eq., $\mathrm{M}=98.15, \mathrm{~d}=0.89)$ of 2-hexyn-1-ol, 2.3 $\mathrm{mL}(22.5 \mathrm{mmol}, 1.5 \mathrm{eq}, \mathrm{M}=135, \mathrm{~d}=1.34)$ of 1-bromo-2-methyl-prop-2-ene and $432 \mathrm{mg}(18 \mathrm{mmol}, 1.2 \mathrm{eq}, \mathrm{M}=24)$ of sodium hydride in DMF (0.67 M), the 1-((2-methylallyl)oxy)hex-2-yne [1i] was obtained as an Colorless oil (1.53 g, 67 \%).

${ }^{1} \mathrm{H}$ NMR $\left(\mathrm{CDCl}_{3}, 200 \mathrm{MHz} ; \delta\right.$ ppm) :

$5.02-4.96\left(\mathrm{~m}, 1 \mathrm{H}, \mathbf{6}^{\prime}\right), 4.92(\mathrm{dd}, J=1.4,0.7 \mathrm{~Hz}, 1 \mathrm{H}, \mathbf{6}$ ''), $4.12(\mathrm{t}, J=2.2 \mathrm{~Hz}, 2 \mathrm{H}, 2), 3.96$ (s, 2H, 5), 2.21 (tt, $J=7.0,2.1 \mathrm{~Hz}, 2 \mathrm{H}$, 8), $1.75(\mathrm{~s}, 3 \mathrm{H}, 9), 1.54(\mathrm{q}, J=7.3 \mathrm{~Hz}, 2 \mathrm{H}, \mathbf{1 0}), 0.99(\mathrm{t}, J=7.3 \mathrm{~Hz}, 3 \mathrm{H}, 11)$.

${ }^{13} \mathrm{C}$ NMR $\left(\mathrm{CDCl}_{3}, 50 \mathrm{MHz} ; \delta\right.$ ppm) :

$141.73(\mathrm{C}, 4), 112.73\left(\mathrm{CH}_{2}, \mathbf{6}\right), 86.80(\mathrm{C}, \mathbf{3}), 76.07(\mathrm{C}, 7), 73.40\left(\mathrm{CH}_{2}, \mathbf{5}\right), 57.57\left(\mathrm{CH}_{2}, 2\right), 22.07\left(\mathrm{CH}_{2}, 10\right), 20.77\left(\mathrm{CH}_{2}, \mathbf{8}\right), 19.56$ $\left(\mathrm{CH}_{3}, 9\right), 13.49\left(\mathrm{CH}_{3}, \mathbf{1 1}\right)$.

HRMS (EI) $\mathbf{m} / \mathbf{z}:[\mathrm{M}+\mathrm{H}]^{+}$Calcd for $\mathrm{C}_{10} \mathrm{H}_{16} \mathrm{O}$ 153.1279; Found 153.1267

1-((2-methylallyl)oxy)hept-2-yne [1j]

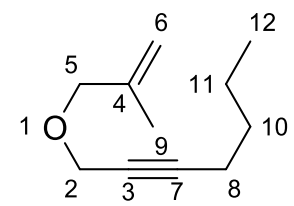
Formula : $\mathrm{C}_{11} \mathrm{H}_{18} \mathrm{O}$
$\mathrm{M}: 166.26$ g.mol $^{-1}$
Aspect : Light yellow oil
$\mathrm{R}_{\mathrm{f}}=0.63$ (petroleum ether/ $\mathrm{Et}_{2} \mathrm{O}, 96 / 4 ; \mathrm{KMnO}_{4}$ )
$\mathrm{GC}: 6.72 \mathrm{~min}$

According to the experimental procedure (1) and starting from $1 \mathrm{~mL}(8 \mathrm{mmol}, 1$ eq., $\mathrm{M}=112.2, \mathrm{~d}=0.88)$ of hept-2-yn-1-ol, 1.2 $\mathrm{mL}(12 \mathrm{mmol}, 1.5 \mathrm{eq}, \mathrm{M}=135, \mathrm{~d}=1.34)$ of 3-bromo-2-methylprop-1-ene and $230 \mathrm{mg}(9.6 \mathrm{mmol}, 1.2 \mathrm{eq}, \mathrm{M}=24)$ of sodium hydride in DMF (0.67 M), the 1-((2-methylallyl)oxy)hept-2-yne [1j] was obtained as a yellow oil (1.2 g, 90 \%).

${ }^{1} \mathrm{H}$ NMR $\left(\mathrm{CDCl}_{3}, 400 \mathrm{MHz} ; \delta\right.$ ppm) :

$4.98\left(\mathrm{dq}, J=2.2,1.1 \mathrm{~Hz}, 1 \mathrm{H}, \mathbf{6}^{\prime}\right), 4.91\left(\mathrm{t}, J=1.8 \mathrm{~Hz}, 1 \mathrm{H}, \mathbf{6}^{\prime}\right.$ ') $, 4.11(\mathrm{t}, J=2.1 \mathrm{~Hz}, 2 \mathrm{H}, 2), 3.96$ (s, 2H, 5), 2.23 (tt, $J=7.1,2.2 \mathrm{~Hz}$, $2 \mathrm{H}, \mathbf{8}), 1.75(\mathrm{~s}, 3 \mathrm{H}, 9), 1.56-1.35(\mathrm{~m}, 4 \mathrm{H}, \mathbf{1 0}, \mathbf{1 1}), 0.91(\mathrm{t}, J=7.2 \mathrm{~Hz}, 3 \mathrm{H}, 12)$.

${ }^{13} \mathrm{C}$ NMR $\left(\mathrm{CDCl}_{3}, 101 \mathrm{MHz} ; \delta\right.$ ppm) :

$141.73(\mathrm{C}, 4), 112.73\left(\mathrm{CH}_{2}, \mathbf{6}\right), 86.94(\mathrm{C}, 7), 75.90(\mathrm{C}, \mathbf{3}), 73.41\left(\mathrm{CH}_{2}, \mathbf{5}\right), 57.59\left(\mathrm{CH}_{2}, 2\right), 30.70\left(\mathrm{CH}_{2}, 10\right), 21.94\left(\mathrm{CH}_{2}, 11\right), 19.56$ $\left(\mathrm{CH}_{3}, 9\right), 18.46\left(\mathrm{CH}_{2}, \mathbf{8}\right), 13.58\left(\mathrm{CH}_{3}, 12\right)$.

HRMS (EI) m/z : $[\mathrm{M}+\mathrm{H}]^{+}$Calcd for $\mathrm{C}_{11} \mathrm{H}_{18} \mathrm{O}$ 167.1436; Found 167.142 


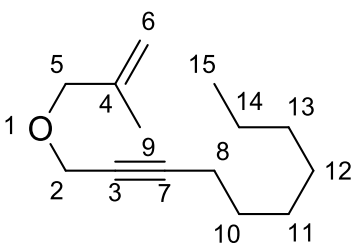

\author{
Formula : $\mathrm{C}_{14} \mathrm{H}_{24} \mathrm{O}$ \\ $\mathrm{M}: 208.35$ g.mol ${ }^{-1}$ \\ Aspect : Colorless oil \\ $\mathrm{R}_{\mathrm{f}}=0.68$ (petroleum ether $/ \mathrm{Et}_{2} \mathrm{O}, 96 / 4 ; \mathrm{KMnO}_{4}$ ) \\ $\mathrm{GC}: 10.29 \mathrm{~min}$
}

According to the experimental procedure (1) and starting from $2.8 \mathrm{~mL}$ (15 mmol, 1 eq., $\mathrm{M}=154.25$, d= 0.85) of 2-decyn-1-ol, 2.3 $\mathrm{mL}$ (22.5 mmol, $1.5 \mathrm{eq}, \mathrm{M}=135, \mathrm{~d}=1.34)$ of 1-bromo-2-methyl-prop-2-ene and $432 \mathrm{mg}(18 \mathrm{mmol}, 1.2 \mathrm{eq}, \mathrm{M}=24) \mathrm{of}$ sodium hydride in DMF (0.67 M), the 1-((2-methylallyl)oxy)dec-2-yne [1I] was obtained as an Colorless oil (3.12 g, $100 \%)$.

Distillation : $94^{\circ} \mathrm{C}$ at $1.5 \mathrm{mBar}$

${ }^{1} \mathrm{H}$ NMR $\left(\mathrm{CDCl}_{3}, 200 \mathrm{MHz} ; \delta\right.$ ppm) :

$4.98(\mathrm{dd}, J=2.0,1.0 \mathrm{~Hz}, 2 \mathrm{H}, \mathbf{6}$ ') $4.97-4.87$ (m, 1H, 6' ) $, 4.11(\mathrm{t}, J=2.2 \mathrm{~Hz}, 2 \mathrm{H}, 2), 3.96(\mathrm{~s}, 2 \mathrm{H}, \mathbf{5}), 2.22(\mathrm{tt}, J=6.9,2.1 \mathrm{~Hz}, 3 \mathrm{H}$, 8), $1.75(\mathrm{~s}, 4 \mathrm{H}, 9), 1.56-1.20(\mathrm{~m}, 10 \mathrm{H}, \mathbf{1 0}, \mathbf{1 1}, \mathbf{1 2}, \mathbf{1 3}, \mathbf{1 4}), 0.96-0.80(\mathrm{~m}, 3 \mathrm{H}, 15)$.

${ }^{13} \mathrm{C}$ NMR $\left(\mathrm{CDCl}_{3}, 50 \mathrm{MHz} ; \delta\right.$ ppm) :

$141.73(\mathrm{C}, 4), 112.70\left(\mathrm{CH}_{2}, \mathbf{6}\right), 87.02(\mathrm{C}, 7), 75.91(\mathrm{C}, \mathbf{3}), 73.39\left(\mathrm{CH}_{2}, \mathbf{5}\right), 57.59\left(\mathrm{CH}_{2}, 2\right), 31.74\left(\mathrm{CH}_{2}, 13\right), 28.83\left(\mathrm{CH}_{2}, 12\right), 28.80$ $\left(\mathrm{CH}_{2}, \mathbf{1 1}\right), 28.64\left(\mathrm{CH}_{2}, \mathbf{1 0}\right), 22.62\left(\mathrm{CH}_{2}, \mathbf{1 4}\right), 19.56\left(\mathrm{CH}_{3}, 9\right), 18.78\left(\mathrm{CH}_{2}, \mathbf{8}\right), 14.07\left(\mathrm{CH}_{2}, 15\right)$.

HRMS (EI) $\mathbf{m} / \mathbf{z}:[\mathrm{M}+\mathrm{H}]^{+}$Calcd for $\mathrm{C}_{14} \mathrm{H}_{24} \mathrm{O}$ 209.1905; Found 209.1902

Synthesis of the bis ether derivatives<smiles>C=C(C)CBr</smiles><smiles>O=S(=O)(O[Na])O[Na]</smiles>
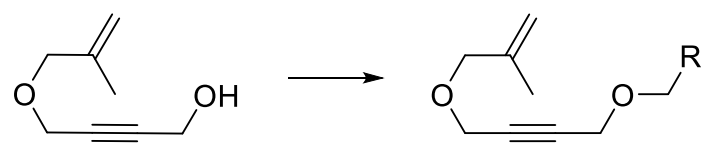

2-butyne-1,4-diol (182.5 mmol, $2.5 \mathrm{eq}, 16.14 \mathrm{~g}$ ) and KOH (182.5 mmol, $2.5 \mathrm{eq}, 10.52 \mathrm{~g}$ ) were dissolved in dry DMSO (1.65 M, $120 \mathrm{~mL})$ under argon. After $30 \mathrm{~min}$ of stirring at r.t. alkyl bromide $(75 \mathrm{mmol}, 1 \mathrm{eq}, 7.6 \mathrm{~mL})$ were added. Then, the reaction mixture was stirred for $60 \mathrm{~h}$ at r.t. The solution was diluted with $\mathrm{CH}_{2} \mathrm{Cl}_{2}$ and washed with $100 \mathrm{~mL}$ of a $1 \mathrm{M} \mathrm{HCl}$ solution, then sat. $\mathrm{Na}_{2} \mathrm{CO}_{3}$ solution and finally with brine. The combined org. layers were dried over $\mathrm{MgSO}_{4}$ and filtered. The filtrate was concentrated and purified by flash column chromatography using silica gel. (80:20/ Petroleum Ether:Et $\left.{ }_{2} \mathrm{O}\right)$ to obtain titled compound 4-((2-methylallyl)oxy)but-2-yn-1-ol [1n'] as a colorless oil (10.51 g, 69\%) and 1,4-bis((2-methylallyl)oxy)but-2-yne [1n] as a colorless oil $(900 \mathrm{mg}, 6 \%)$.

tert-butyl 2-((4-((2-methylallyl)oxy)but-2-yn-1-yl)oxy)acetate [10]

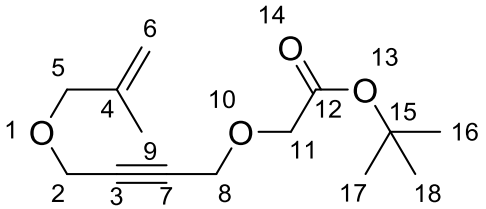

$$
\begin{aligned}
& \text { Formula }: \mathrm{C}_{14} \mathrm{H}_{22} \mathrm{O}_{4} \\
& \mathrm{M}: 254.33 \mathrm{~g} \cdot \mathrm{mol}^{-1} \\
& \text { Aspect }: \text { Colorless oil } \\
& \mathrm{R}_{\mathrm{f}}=0.29 \text { (petroleum ether } / \mathrm{Et}_{2} \mathrm{O}, 90 / 10 ; \mathrm{KMnO}_{4} \text { ) } \\
& \mathrm{GC}: 12.19 \text { min }
\end{aligned}
$$

According to the experimental procedure (2) and starting from a suspension of NaH (1.5 eq, $4.3 \mathrm{mmol}, 103 \mathrm{mg})$ in $\mathbf{T H F}(0.5 \mathrm{M})$ at $0^{\circ} \mathrm{C}$, the alcohol [1n'] (1 eq, $\left.2.9 \mathrm{mmol}, 400 \mathrm{mg}\right)$, tert-butyl 2-bromoacetate ( $\left.2 \mathrm{eq}, 5.7 \mathrm{mmol}, 840 \mu \mathrm{L}\right)$, the tert-butyl 2-((4-((2methylallyl)oxy)but-2-yn-1-yl)oxy)acetate [10] was obtained as an incolour oil (393 mg, $54 \%$ ).

${ }^{1} \mathrm{H}$ NMR $\left(\mathrm{CDCl}_{3}, 400 \mathrm{MHz} ; \delta\right.$ ppm) :

$4.99(\mathrm{dd}, J=2.1,1.1 \mathrm{~Hz}, 1 \mathrm{H}, \mathbf{6}), 4.93(\mathrm{t}, J=1.8 \mathrm{~Hz}, 1 \mathrm{H}, \mathbf{6}), 4.35$ (t, $J=1.8 \mathrm{~Hz}, 2 \mathrm{H}, 8), 4.18(\mathrm{t}, J=1.8 \mathrm{~Hz}, 2 \mathrm{H}, 2), 4.07(\mathrm{~s}, 2 \mathrm{H}$, 11), $3.96(\mathrm{~s}, 2 \mathrm{H}, \mathbf{5}), 1.75$ (s, 2H, 9), 1.49 (s, 9H, 16, 17, 18).

${ }^{13} \mathrm{C}$ NMR $\left(\mathrm{CDCl}_{3}, 101 \mathrm{MHz} ; \delta \mathrm{ppm}\right)$ :

$169.07(\mathrm{C}=0,12), 141.37(\mathrm{C}, 4), 113.02\left(\mathrm{CH}_{2}, \mathbf{6}\right), 83.31(\mathrm{C}, \mathbf{1 5}), 81.84(\mathrm{C}, 7), 81.25(\mathrm{C}, \mathbf{3}), 73.71\left(\mathrm{CH}_{2}, 5\right), 66.66\left(\mathrm{CH}_{2}, 11\right), 58.38$ $\left(\mathrm{CH}_{2}, \mathbf{2}\right), 57.19\left(\mathrm{CH}_{2}, \mathbf{8}\right), 28.09\left(\mathrm{CH}_{3}, \mathbf{1 6}, \mathbf{1 7}, \mathbf{1 8}\right), 19.50\left(\mathrm{CH}_{3}, 9\right)$.

HRMS (EI) $\mathbf{m} / \mathbf{z}:[\mathrm{M}+\mathrm{H}]^{+}$Calcd for $\mathrm{C}_{14} \mathrm{H}_{22} \mathrm{O}_{4}$ 255.1596; Found 255.1587 


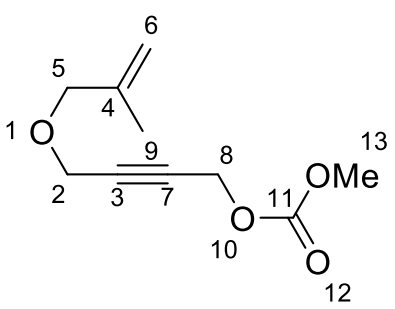

\author{
Formula : $\mathrm{C}_{10} \mathrm{H}_{14} \mathrm{O}_{4}$ \\ $\mathrm{M}: 198.22 \mathrm{~g} \cdot \mathrm{mol}^{-1}$ \\ Aspect : Colorless oil \\ $\mathrm{R}_{\mathrm{f}}=0.84$ (petroleum ether/ $\mathrm{Et}_{2} \mathrm{O}, 60 / 40 ; \mathrm{KMnO}_{4}$ )
}

According to the experimental procedure (1) and starting from a suspension of NaH (1.5 eq, $5.4 \mathrm{mmol}, 128 \mathrm{mg})$ in $\mathrm{THF}(0.5 \mathrm{M})$ at $0^{\circ} \mathrm{C}$, the alcohol [1n'] (1 eq, $\left.4.3 \mathrm{mmol}, 600 \mathrm{mg}\right)$, methyl chlorofomate $(2 \mathrm{eq}, 7.1 \mathrm{mmol}, 0.55 \mathrm{~mL})$, the methyl (4-((2methylallyl)oxy)but-2-yn-1-yl) carbonate [1 m] was obtained as a colorless oil (382 mg, $54 \%$ ).

${ }^{1} \mathrm{H}$ NMR $\left(\mathrm{CDCl}_{3}, 400 \mathrm{MHz} ; \delta\right.$ ppm) :

$4.98\left(\mathrm{dq}, J=2.2,1.1 \mathrm{~Hz}, 1 \mathrm{H}, 6^{\prime}\right), 4.93\left(\mathrm{t}, J=1.8 \mathrm{~Hz}, 1 \mathrm{H}, 6^{\prime \prime}\right), 4.79(\mathrm{t}, J=1.8 \mathrm{~Hz}, 2 \mathrm{H}, 8), 4.17(\mathrm{t}, J=1.8 \mathrm{~Hz}, 2 \mathrm{H}, 2), 3.96(\mathrm{~s}, 2 \mathrm{H}, 5), 3.82$ $(\mathrm{s}, 3 \mathrm{H}, 14), 1.75(\mathrm{t}, J=1.2 \mathrm{~Hz}, 3 \mathrm{H}, 9)$.

${ }^{13} \mathrm{C}$ NMR $\left(\mathrm{CDCl}_{3}, 101 \mathrm{MHz} ; \delta\right.$ ppm) :

$155.20(\mathrm{C}=\mathrm{O}, 11), 141.30(\mathrm{C}=, 4), 113.09\left(=\mathrm{CH}_{2}, 6\right), 83.72(\mathrm{C}, 7), 79.58(\mathrm{C}, 3), 73.72\left(\mathrm{CH}_{2}, 5\right), 57.07\left(\mathrm{CH}_{2}, 2\right), 55.65\left(\mathrm{CH}_{2}, 8\right), 55.13$ $\left(\mathrm{CH}_{3}, 13\right), 19.48\left(\mathrm{CH}_{3}, 9\right)$.

HRMS (EI) m/z : [M+H $]^{+}$Calcd for $\mathrm{C}_{10} \mathrm{H}_{14} \mathrm{O}$ 199.0970; Found 199.0977

\title{
Synthesis of substituted alkene
}

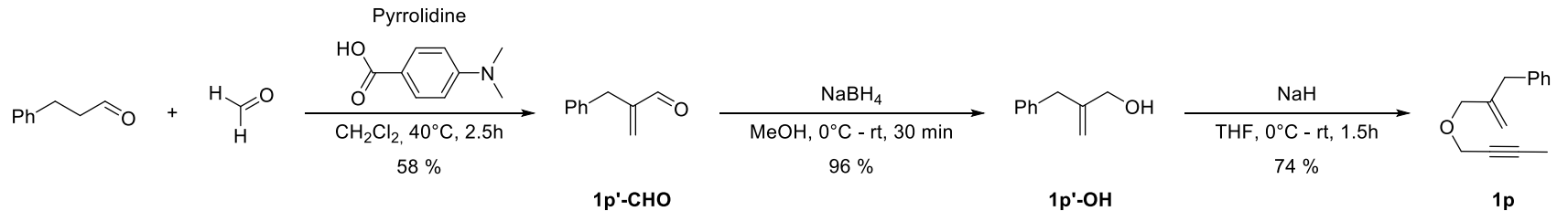

(2-((but-2-yn-1-yloxy)methyl)allyl)benzene [1p]

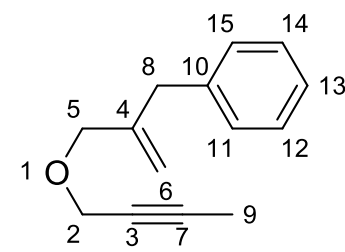

\author{
Formula : $\mathrm{C}_{14} \mathrm{H}_{16} \mathrm{O}$ \\ $\mathrm{M}: 200.28$ g.mol ${ }^{-1}$ \\ Aspect : Colorless oil \\ $\mathrm{R}_{\mathrm{f}}=0.63$ (Pentane $/ \mathrm{Et}_{2} \mathrm{O}, 90 / 10 ;$ Kagi)
}

According to the experimental procedure (1) and starting from a suspension of NaH (1.2 eq, $4.7 \mathrm{mmol}, 113 \mathrm{mg})$ in THF (0.5 M) at $0^{\circ} \mathrm{C}$, the alcohol [1p'-OH] (1 eq, $\left.3.9 \mathrm{mmol}, 580 \mathrm{mg}\right)$, 1-Bromo-2-butyne (1.5 eq, $\left.5.9 \mathrm{mmol}, 0.5 \mathrm{~mL}\right)$, the (2-((but-2-yn-1yloxy)methyl)allyl)benzene [1p] was obtained as a colorless oil (579 $\mathrm{mg}, 74 \%)$.

${ }^{1} \mathrm{H}$ NMR $\left(\mathrm{CDCl}_{3}, 400 \mathrm{MHz} ; \delta\right.$ ppm) :

$7.33-7.25$ (m, 2H, 11, 15), $7.24-7.15(\mathrm{~m}, 3 \mathrm{H}, \mathbf{1 2}, \mathbf{1 3}, \mathbf{1 4}), 5.11\left(\mathrm{~s}, 1 \mathrm{H}, \mathbf{6}^{\prime}\right), 4.92\left(\mathrm{~d}, \mathrm{~J}=2.0 \mathrm{~Hz}, 1 \mathrm{H}, \mathbf{6}^{\prime}\right.$ '), $4.06(\mathrm{q}, \mathrm{J}=2.3 \mathrm{~Hz}, 2 \mathrm{H}$, 2), $3.92(\mathrm{~s}, 2 \mathrm{H}, \mathbf{5}), 3.40(\mathrm{~s}, 2 \mathrm{H}, \mathbf{8}), 1.91-1.81(\mathrm{~m}, 3 \mathrm{H}, \mathbf{9})$.

${ }^{13} \mathrm{C}$ NMR $\left(\mathrm{CDCl}_{3}, 101 \mathrm{MHz} ; \delta \mathrm{ppm}\right)$ :

$145.02(\mathrm{C}, \mathbf{4}), 139.08(\mathrm{C}, \mathbf{1 0}), 129.09(2 \times \mathrm{CH}, \mathbf{1 2}, \mathbf{1 4}), 128.30(2 \times \mathrm{CH}, \mathbf{1 1}, \mathbf{1 5}), 126.14(\mathrm{CH}, \mathbf{1 3}), 113.97\left(\mathrm{CH}_{2}, \mathbf{6}\right), 82.39(\mathrm{C}, 7)$, $75.12(\mathrm{C}, \mathbf{3}), 71.77\left(\mathrm{CH}_{2}, \mathbf{5}\right), 57.70\left(\mathrm{CH}_{2}, \mathbf{2}\right), 39.89\left(\mathrm{CH}_{2}, \mathbf{8}\right), 3.60\left(\mathrm{CH}_{3}, \mathbf{9}\right)$.

HRMS (EI) m/z: $[\mathrm{M}+\mathrm{H}]^{+}$Calcd for $\mathrm{C}_{14} \mathrm{H}_{16} \mathrm{O}$ 201.1279; Found 201.1270 


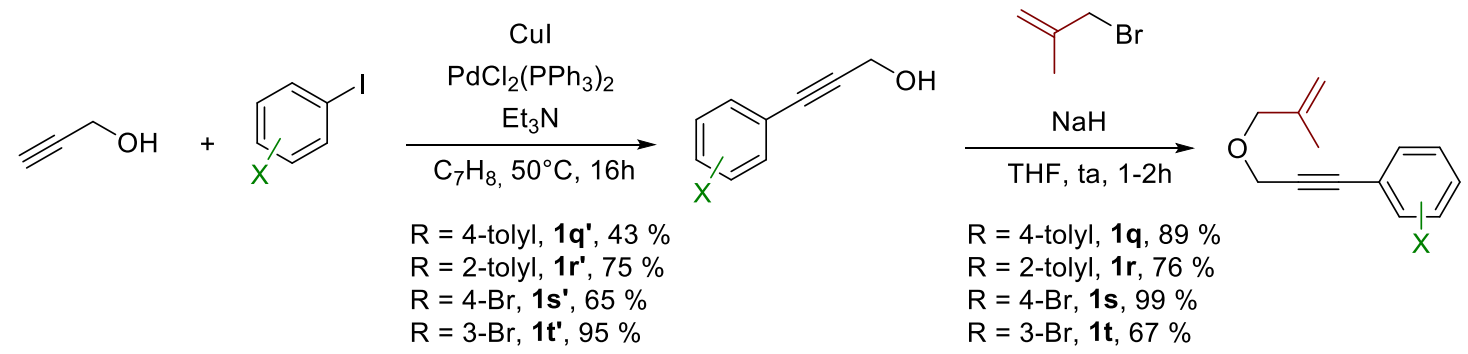

1-bromo-4-(3-((2-methylallyl)oxy)prop-1-yn-1-yl)benzene [1s]

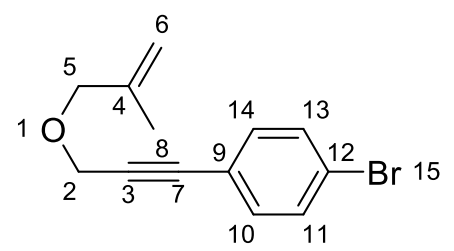

$$
\begin{aligned}
& \text { Formula : } \mathrm{C}_{13} \mathrm{H}_{13} \mathrm{BrO} \\
& \mathrm{M}: 265.15 \mathrm{~g} \cdot \mathrm{mol}^{-1} \\
& \text { Aspect }: \text { Light yellow oil } \\
& \mathrm{R}_{\mathrm{f}}=0.79\left(\text { Pentane } / \mathrm{Et}_{2} \mathrm{O}, 80 / 20 ; \mathrm{KMnO}_{4}\right)
\end{aligned}
$$

According to the experimental procedure (1) and starting from $1.5 \mathrm{~g}(7.1 \mathrm{mmol}, 1 \mathrm{eq}$.$) of [1s'], 1.07 \mathrm{~mL}(10.7 \mathrm{mmol}, 1.5 \mathrm{eq}, \mathrm{M}=$ 135) of 3-bromo-2-methylprop-1-ene and $205 \mathrm{mg}(8.5 \mathrm{mmol}, 1.2 \mathrm{eq}, \mathrm{M}=24)$ of sodium hydride in THF (0.67 $\mathrm{M})$, the 1bromo-4-(3-((2-methylallyl)oxy)prop-1-yn-1-yl)benzene [1s] was obtained as a yellow oil (1.87 g, 99 \%).

${ }^{1} \mathrm{H}$ NMR $\left(\mathrm{CDCl}_{3}, 400 \mathrm{MHz} ; \delta\right.$ ppm) :

$7.48-7.41(\mathrm{~m}, 2 \mathrm{H}, \mathbf{1 1}, \mathbf{1 3}), 7.34-7.26(\mathrm{~m}, 2 \mathrm{H}, \mathbf{1 0}, \mathbf{1 4}), 5.02(\mathrm{dq}, J=2.2,1.1 \mathrm{~Hz}, 1 \mathrm{H}, \mathbf{6}$ '’), 4.95 (t, $J=1.8 \mathrm{~Hz}, 1 \mathrm{H}, \mathbf{6}$ '), 4.33 (s, 2H, 2), $4.03(\mathrm{~s}, 2 \mathrm{H}, \mathbf{5}), 1.78(\mathrm{t}, J=1.2 \mathrm{~Hz}, 3 \mathrm{H}, \mathbf{8})$.

${ }^{13} \mathrm{C}$ NMR $\left(\mathrm{CDCl}_{3}, 101 \mathrm{MHz} ; \delta\right.$ ppm) :

141.45 (C, 4), 133.18 (2xCH, 11, 13), 131.55 (2xCH, 10, 14), $122.67(\mathrm{C}, \mathbf{1 2}), 121.65(\mathrm{C}, \mathbf{9}), 113.10\left(\mathrm{CH}_{2}, \mathbf{6}\right), 86.41(\mathrm{C}, 7), 85.04$ $(\mathrm{C}, \mathbf{3}), 73.84\left(\mathrm{CH}_{2}, \mathbf{5}\right), 57.70\left(\mathrm{CH}_{2}, 2\right), 19.55\left(\mathrm{CH}_{3}, \mathbf{8}\right)$.

HRMS (EI) m/z : $[\mathrm{M}+\mathrm{H}]^{+}$Calcd for $\mathrm{C}_{13} \mathrm{H}_{13} \mathrm{BrO} 265.0228$; Found 265.0218

1-bromo-3-(3-((2-methylallyl)oxy)prop-1-yn-1-yl)benzene [1t]

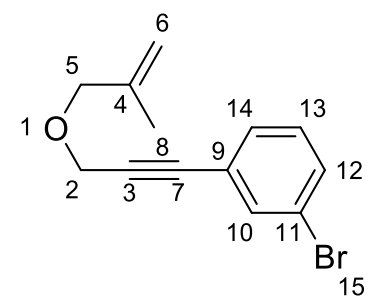

Formula : $\mathrm{C}_{13} \mathrm{H}_{13} \mathrm{BrO}$

$\mathrm{M}: 265.15 \mathrm{~g} \cdot \mathrm{mol}^{-1}$

Aspect : Light yellow oil

$\mathrm{R}_{\mathrm{f}}=0.61$ (petroleum ether/ $\mathrm{Et}_{2} \mathrm{O}, 96 / 4 ; \mathrm{KMnO}_{4}$ )

$\mathrm{GC}: 13.67 \mathrm{~min}$

According to the experimental procedure (1) and starting from $600 \mathrm{mg}(2.8 \mathrm{mmol}, 1$ eq.) of [1t'], $430 \mu \mathrm{L}(4.3 \mathrm{mmol}, 1.5 \mathrm{eq}, \mathrm{M}=$ 135) of 3-bromo-2-methylprop-1-ene and $82 \mathrm{mg}(3.4 \mathrm{mmol}, 1.2 \mathrm{eq}, \mathrm{M}=24)$ of sodium hydride in DMF (0.67 M), the 1bromo-3-(3-((2-methylallyl)oxy)prop-1-yn-1-yl)benzene [1t] was obtained as a yellow oil (680 mg, $90 \%)$.

${ }^{1} \mathrm{H}$ NMR $\left(\mathrm{CDCl}_{3}, 400 \mathrm{MHz} ; \delta\right.$ ppm) :

${ }^{1} \mathrm{H}$ NMR $(400 \mathrm{MHz}, 3) \delta 7.60(\mathrm{t}, J=1.6 \mathrm{~Hz}, 1 \mathrm{H}, \mathbf{1 0}), 7.45(\mathrm{ddd}, J=8.1,1.8,1.0 \mathrm{~Hz}, 1 \mathrm{H}, \mathbf{1 2}), 7.40-7.34(\mathrm{~m}, 1 \mathrm{H}, 14), 7.18(\mathrm{t}, J=$ $7.9 \mathrm{~Hz}, 1 \mathrm{H}, 13), 5.07-4.99$ (m, 2H, 6' '), 4.96 (s, 1H, 6'), 4.35 (s, 1H, 2), 4.03 (s, 2H, 5), 1.78 (s, 3H, 8).

${ }^{13} \mathrm{C}$ NMR $\left(\mathrm{CDCl}_{3}, 101 \mathrm{MHz} ; \delta\right.$ ppm) :

${ }^{13} \mathrm{C}$ NMR $\left(101 \mathrm{MHz}, \mathrm{CDCl}_{3}\right) \delta 141.45(\mathrm{C}, 4), 134.50(\mathrm{CH}, \mathbf{1 0}), 131.60(\mathrm{CH}, \mathbf{1 2}), 130.31(\mathrm{CH}, \mathbf{1 4}), 129.74(\mathrm{CH}, 13), 124.71(\mathrm{C}$, 9), $122.09(\mathrm{C}, \mathbf{1 1}), 113.15\left(\mathrm{CH}_{2}, \mathbf{6}\right), 86.65(\mathrm{C}, \mathbf{3}), 84.58(\mathrm{C}, 7), 73.84\left(\mathrm{CH}_{2}, \mathbf{5}\right), 57.63\left(\mathrm{CH}_{2}, 2\right), 19.57\left(\mathrm{CH}_{3}, 8\right)$.

HRMS (EI) m/z: $[\mathrm{M}+\mathrm{H}]^{+}$Calcd for $\mathrm{C}_{13} \mathrm{H}_{13} \mathrm{BrO} 265.0228$; Found 265.0219 


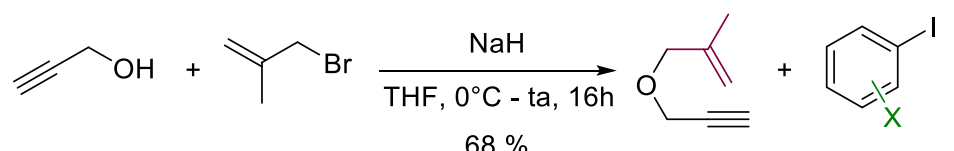
$68 \%$

4-(3-((2-methylallyl)oxy)prop-1-yn-1-yl)benzonitrile [1v]<smiles>C=C(C)COCC#Cc1ccc(C#N)cc1</smiles>

Formula : $\mathrm{C}_{14} \mathrm{H}_{13} \mathrm{NO}$ $\mathrm{M}: 211.26 \mathrm{~g} \cdot \mathrm{mol}^{-1}$ Aspect : Colorless oil $\mathrm{R}_{\mathrm{f}}=0.53$ (petroleum ether/Et ${ }_{2} \mathrm{O}, 80 / 20$; Kagi-Mosher)

To a suspension of Copper Iodide ( $1 \mathrm{~mol} \%, 34 \mu \mathrm{mol}, 6 \mathrm{mg})$, Palladium-dichloro-bis(triphenylphosphine) $(2 \mathrm{~mol} \%, 68 \mu \mathrm{mol}$, $48 \mathrm{mg}$ ), ether [1'] (1 eq, $3.4 \mathrm{mmol}, 375 \mathrm{mg})$ in $\mathbf{E t}_{3} \mathbf{N}(1 \mathrm{M})$ and Toluene $(0.33 \mathrm{M})$ is added 4-iodo-benzonitrile (1.1 eq, 3.7 mmol, $681 \mathrm{mg}$ ). Reaction mixture is stirred until completion of the reaction monitored by TLC. Reaction mixture is diluted in ca. $30 \mathrm{~mL}$ of water, extracted thrice with EtOAc. Combined organic phase are washed with brine, dried over $\mathrm{MgSO}_{4}$ then concentrated in vacuuo. Crude mixture is purified on silica column to afford title compound 4-(3-((2-methylallyl)oxy)prop-1-yn1-yl)benzonitrile [1v] as an Colorless oil (278 $\mathrm{mg}, 39 \%)$

${ }^{1} \mathrm{H}$ NMR $\left(\mathrm{CDCl}_{3}, 400 \mathrm{MHz} ; \delta\right.$ ppm) :

$7.61(\mathrm{~d}, \mathrm{~J}=8.5 \mathrm{~Hz}, 2 \mathrm{H}, \mathbf{7}, \mathbf{9}), 7.56-7.49(\mathrm{~m}, 2 \mathrm{H}, \mathbf{6}, \mathbf{1 0}), 4.97(\mathrm{t}, \mathrm{J}=1.8 \mathrm{~Hz}, 1 \mathrm{H}, \mathbf{1 3}), 4.37(\mathrm{~s}, 2 \mathrm{H}, \mathbf{1}), 4.03(\mathrm{~s}, 2 \mathrm{H}, 11), 1.78(\mathrm{t}, \mathrm{J}=$ $1.2 \mathrm{~Hz}, 3 \mathrm{H}, 14)$.

${ }^{13} \mathrm{C}$ NMR (CDCl $3,101 \mathrm{MHz} ; \delta$ ppm) :

$141.28(\mathrm{C}, 12), 132.23(2 \times C H, 7,9), 132.00(2 \times C H, 6,10), 127.61(\mathrm{C}, 5), 118.38(\mathrm{C}, 15), 113.29\left(\mathrm{CH}_{2}, 13\right), 111.83(\mathrm{C}, 8), 89.87(\mathrm{C}, 4)$, $84.46(\mathrm{C}, 3), 74.05\left(\mathrm{CH}_{2}, 11\right), 57.57\left(\mathrm{CH}_{2}, 1\right), 19.52\left(\mathrm{CH}_{3}, 14\right)$.

HRMS (EI) m/z: $[\mathrm{M}+\mathrm{H}]^{+}$Calcd for $\mathrm{C}_{14} \mathrm{H}_{13} \mathrm{NO} 212.1075$; Found: 212.1071

\section{Cycloisomerization of synthesized enynes}

7,7-dimethyl-6-phenyl-3-oxabicyclo[4.1.0]hept-4-ene [2a]

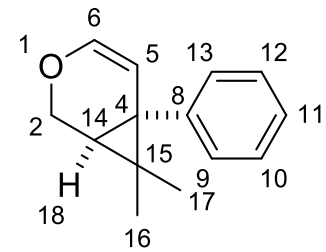

16
Formula : $\mathrm{C}_{14} \mathrm{H}_{16} \mathrm{O}$

$\mathrm{M}: 200.28$ g.mol ${ }^{-1}$

Aspect : Colorless oil

[1a], $7 \mathrm{mg}(11 \mathrm{umol} 2 \mathrm{~mol} \%$ ) oxabicyclo[4.1.0]hept-4-ene [2a] was obtained after $1 \mathrm{~h}$ of reaction as an uncolored oil (101 $\mathrm{mg}, 90 \%)$.

${ }^{1} \mathrm{H}$ NMR $\left(\mathrm{CDCl}_{3}, 400 \mathrm{MHz} ; \delta\right.$ ppm) :

$7.35-7.27(\mathrm{~m}, 2 \mathrm{H}, 9,13), 7.26-7.16(\mathrm{~m}, 3 \mathrm{H}, 10,11,12), 6.23(\mathrm{~d}, J=6.2 \mathrm{~Hz}, 1 \mathrm{H}, 6), 4.97(\mathrm{dd}, J=6.2,1.1 \mathrm{~Hz}, 1 \mathrm{H}, 5), 4.21(\mathrm{~d}, J=$ $2.9 \mathrm{~Hz}, 2 \mathrm{H}, 2), 1.50(\mathrm{td}, J=2.9,1.0 \mathrm{~Hz}, 1 \mathrm{H}, 18), 1.22(\mathrm{~s}, 3 \mathrm{H}, 17), 0.79(\mathrm{~s}, 3 \mathrm{H}, 16)$.

${ }^{13} \mathrm{C}$ NMR $\left(\mathrm{CDCl}_{3}, 101 \mathrm{MHz} ; \delta \mathrm{ppm}\right)$ :

$143.30(\mathrm{CH}, 6), 139.69(\mathrm{CH}, 8), 128.82(2 \times \mathrm{CH}, 10,12), 128.32(2 \times \mathrm{CH}, 9,13), 126.02(\mathrm{CH}, 11), 105.84(\mathrm{CH}, 5), 60.80(\mathrm{CH}, 2), 29.83$ (C, 15), $29.17(\mathrm{CH}, 14), 28.94(\mathrm{C}, 4), 24.81\left(\mathrm{CH}_{3}, 16\right), 16.35\left(\mathrm{CH}_{3}, 17\right)$.

HRMS (EI) m/z: $[\mathrm{M}+\mathrm{H}]^{+}$Calcd for $\mathrm{C}_{14} \mathrm{H}_{17} \mathrm{O}$ 201.12794; Found: 201.12792

6,7-diphenyl-3-oxabicyclo[4.1.0]hept-4-ene [2b]<smiles>CC1C(Pc2ccccc2)[C@H]1CO</smiles> 
the 6,7-diphenyl-3-oxabicyclo[4.1.0]hept-4-ene [2b] was obtained after $1 \mathrm{~h}$ of reaction as a colorless oil (316 $\mathrm{mg}, 77 \%)$.

NMR spectra are in accordance with literature. ${ }^{20,4}$

6-methyl-7-phenyl-3-oxabicyclo[4.1.0]hept-4-ene [2c]<smiles>C[C@]12C=COC[C@H]1[C@H]2c1ccccc1</smiles>

According to the experimental procedure (2) and starting from $214 \mathrm{mg}(1.15 \mathrm{mmol}, 1 \mathrm{eq} ., \mathrm{M}=186.25)$ of [1c], $14 \mathrm{mg}(23 \mu \mathrm{mol}, 2 \mathrm{~mol} \%)$ of IPrAuCl and $20 \mathrm{mg}(23 \mu \mathrm{mol}, 2 \mathrm{~mol} \%)$ of NaBAr F , in Toluene $(0.5 \mathrm{M})$ the 7methyl-6-phenyl-3-oxabicyclo[4.1.0]hept-4-ene [2c] was obtained after $4 \mathrm{~h}$ of reaction as a colorless oil (103 $\mathrm{mg}$, $48 \%$ ).

NMR spectra are in accordance with literature. ${ }^{4}$

7-methyl-6-phenyl-3-oxabicyclo[4.1.0]hept-4-ene [2d]<smiles>C[C@H]1[C@H](c2ccccc2)[C@@H]2C=COC[C@H]12</smiles>

According to the experimental procedure (2) and starting from $300 \mathrm{mg}(1.6 \mathrm{mmol}, 1 \mathrm{eq} ., \mathrm{M}=186.25)$ of 171, 20 $\mathrm{mg}(32 \mu \mathrm{mol}, 2 \mathrm{~mol} \%)$ of IPrAuCl and $29 \mathrm{mg}(32 \mu \mathrm{mol}, 2 \mathrm{~mol} \%)$ of NaBAr ${ }^{\mathrm{F}}$, in Toluene (0.5M) the 7-methyl6-phenyl-3-oxabicyclo[4.1.0]hept-4-ene [2d] was obtained after $3 \mathrm{~h}$ of reaction as a colorless oil (196 mg, $65 \%$ ).

NMR spectra are in accordance with literature. ${ }^{4}$

6-heptyl-3-oxabicyclo[4.1.0]hept-4-ene [2e]

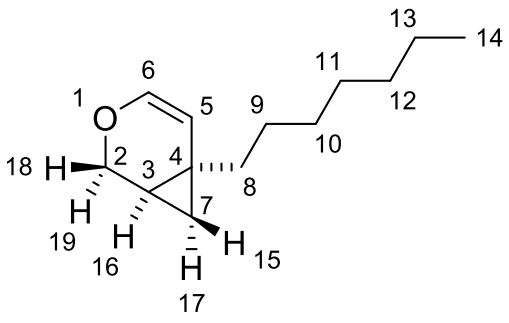

\author{
Formula : $\mathrm{C}_{13} \mathrm{H}_{22} \mathrm{O}$ \\ $\mathrm{M}: 194.32$ g.mol ${ }^{-1}$ \\ Aspect : Colorless oil \\ $\mathrm{R}_{\mathrm{f}}=0.58$ (petroleum ether/ $\mathrm{Et}_{2} \mathrm{O}, 98 / 2 ;$ Kagi-Mosher)
}

According to the experimental procedure (2) and starting from $100 \mathrm{mg}(0.5 \mathrm{mmol}, 1 \mathrm{eq} ., \mathrm{M}=194.32)$ of [1e], $3 \mathrm{mg}(5 \mu \mathrm{mol}, 1$ mol\%) of IPrAuCl and $5 \mathrm{mg}(5 \mu \mathrm{mol}, 1 \mathrm{~mol} \%)$ of $\mathbf{N a B A r}{ }^{\mathrm{F}}$, in Toluene $(0.5 \mathrm{M})$ the 6-heptyl-3-oxabicyclo[4.1.0]hept-4-ene [2e] was obtained after $2 \mathrm{~h}$ of reaction as a colorless oil $(79 \mathrm{mg}, 79 \%)$.

${ }^{1} \mathrm{H}$ NMR $\left(\mathrm{CDCl}_{3}, 400 \mathrm{MHz} ; \delta\right.$ ppm) :

$6.13(\mathrm{~d}, J=6.0 \mathrm{~Hz}, 1 \mathrm{H}, \mathbf{6}), 5.09(\mathrm{dd}, J=6.0,1.0 \mathrm{~Hz}, 1 \mathrm{H}, \mathbf{5}), 4.07(\mathrm{dd}, J=10.4,1.4 \mathrm{~Hz}, 1 \mathrm{H}, \mathbf{1 8}), 3.78(\mathrm{dd}, J=10.4,2.3 \mathrm{~Hz}, 1 \mathrm{H}$, 19), $1.52-1.43(\mathrm{~m}, 1 \mathrm{H}, \mathbf{1 6}), 1.42-1.34(\mathrm{~m}, 2 \mathrm{H}, \mathbf{9}), 1.33-1.24(\mathrm{~m}, 8 \mathrm{H}, \mathbf{1 0}, \mathbf{1 1}, \mathbf{1 2}, \mathbf{1 3}), 1.24-1.10(\mathrm{~m}, 2 \mathrm{H}, \mathbf{8}), 0.91-0.86(\mathrm{~m}$, $3 \mathrm{H}, 14), 0.82(\mathrm{dd}, J=5.4,4.1 \mathrm{~Hz}, 1 \mathrm{H}, 17), 0.71(\mathrm{dd}, J=8.4,4.2,1 \mathrm{H}, 15)$.

${ }^{13} \mathrm{C}$ NMR $\left(\mathrm{CDCl}_{3}, 101 \mathrm{MHz} ; \delta\right.$ ppm) :

$140.93(\mathrm{CH}, \mathbf{6}), 109.37(\mathrm{CH}, \mathbf{5}), 62.65\left(\mathrm{CH}_{2}, \mathbf{2}\right), 37.46\left(\mathrm{CH}_{2}, \mathbf{8}\right), 31.85\left(\mathrm{CH}_{2}, \mathbf{1 2}\right), 29.61\left(\mathrm{CH}_{2}, \mathbf{1 1}\right), 29.35\left(\mathrm{CH}_{2}, \mathbf{1 0}\right), 27.34\left(\mathrm{CH}_{2}\right.$, 7), $24.04(\mathrm{C}, 3), 22.66\left(\mathrm{CH}_{2}, \mathbf{1 3}\right), 19.41\left(\mathrm{CH}_{2}, 9\right), 16.01(\mathrm{C}, \mathbf{4}), 14.09\left(\mathrm{CH}_{3}, 14\right)$.

HRMS (EI) m/z: Calcd for $\mathrm{C}_{13} \mathrm{H}_{22} \mathrm{O}:[\mathrm{M}+\mathrm{H}]^{+} 195.1749$ Found: [M+H $]^{+} 195.1735$

6-phenyl-3-oxabicyclo[4.1.0]hept-4-ene [2f]<smiles>C1=C[C@H]2C[C@H]2COC1</smiles>

According to the experimental procedure (2) and starting from $230 \mathrm{mg}$ (1.34 mmol, 1 eq., $\mathrm{M}=172.23)$ of [1f], 17 $\mathrm{mg}(27 \mu \mathrm{mol}, 2 \mathrm{~mol} \%)$ of IPrAuCl and $34 \mathrm{mg}(27 \mu \mathrm{mol}, 2 \mathrm{~mol} \%)$ of NaBAr ${ }^{\mathbf{F}}$, in Toluene (0.5M) the 6-phenyl3-oxabicyclo[4.1.0]hept-4-ene [2f] was obtained after $4 \mathrm{~h}$ of reaction as a colorless oil (225 $\mathrm{mg}, 98 \%$ ).

NMR spectra are in accordance with literature. ${ }^{4}$

\footnotetext{
${ }^{20}$ Sim, S. H.; Lee, S. I.; Park, J. H.; Chung, Y. K. Adv. Synth. Catal. 2010, 352 (2-3), 317-322.
} 


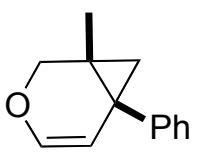

According to the experimental procedure (2) and starting from $8.9 \mathrm{~g}(47.7 \mathrm{mmol}, 1 \mathrm{eq} ., \mathrm{M}=248.33)$ of $[1 \mathrm{~g}], 30$ $\mathrm{mg}(48 \mu \mathrm{mol}, 0.1 \mathrm{~mol} \%)$ of IPrAuCl and $42 \mathrm{mg}(48 \mu \mathrm{mol}, 0.1 \mathrm{~mol} \%)$ of $\mathbf{N a B A r}{ }^{\mathbf{F}}$ in Toluene $(0.5 \mathrm{M})$ the 1methyl-6-phenyl-3-oxabicyclo[4.1.0]hept-4-ene [2g] was obtained after $4 \mathrm{~h}$ of reaction and distillation $\left(81^{\circ} \mathrm{C}\right.$ at $\left.1.7 \mathrm{mBar}\right)$ as a light yellow oil $(8.25 \mathrm{~g}, 93 \%)$.

NMR spectra are in accordance with literature. ${ }^{20}$

6-ethyl-1-methyl-3-oxabicyclo[4.1.0]hept-4-ene [2h]

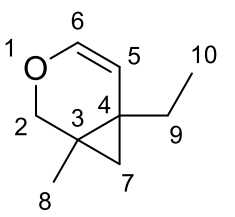

\author{
Formula : $\mathrm{C}_{9} \mathrm{H}_{14} \mathrm{O}$ \\ $\mathrm{M}: 138.21$ g.mol ${ }^{-1}$ \\ Aspect : Colorless oil \\ $\mathrm{R}_{\mathrm{f}}=0.72$ (petroleum ether/Et ${ }_{2} \mathrm{O}, 98 / 2 ;$ Kagi-Mosher)
}

According to the experimental procedure (2) and starting from $398 \mathrm{mg}(2.9 \mathrm{mmol}, 1$ eq., $\mathrm{M}=138.21)$ of [1 h], $4 \mathrm{mg}(6 \mu \mathrm{mol}, 0.2$ mol\%) of IPrAuCl and $5 \mathrm{mg}(6 \mu \mathrm{mol}, 0.2 \mathrm{~mol} \%)$ of NaBAr ${ }^{\mathrm{F}}$, in Toluene (0.5M) the 6-ethyl-1-methyl-3-oxabicyclo[4.1.0]hept4-ene [2h] was obtained after $1.5 \mathrm{~h}$ of reaction as a colorless oil $(293 \mathrm{mg}, 74 \%)$.

${ }^{1} \mathrm{H}$ NMR $\left(\mathrm{CDCl}_{3}, 400 \mathrm{MHz} ; \boldsymbol{\delta} \mathbf{~ p p m}\right)$ :

$6.13(\mathrm{~d}, J=5.8 \mathrm{~Hz}, 1 \mathrm{H}, \mathbf{6}), 5.06(\mathrm{~d}, J=5.8 \mathrm{~Hz}, 1 \mathrm{H}, \mathbf{5}), 3.91\left(\mathrm{~d}, J=10.2 \mathrm{~Hz}, 1 \mathrm{H}, \mathbf{2}^{\prime \prime}\right), 3.47\left(\mathrm{~d}, J=10.1 \mathrm{~Hz}, 1 \mathrm{H}, \mathbf{2}^{\prime}\right), 1.55$ (h, $J=7.5$ $\left.\mathrm{Hz}, 1 \mathrm{H}, 9^{\prime \prime}\right), 1.33$ (dq, $\left.J=13.8,7.0 \mathrm{~Hz}, 1 \mathrm{H}, 9^{\prime}\right), 1.14$ (s, 3H, 8), 1.05 (d, $\left.J=4.0 \mathrm{~Hz}, 1 \mathrm{H}, 7^{\prime}\right), 0.97$ (t, $\left.J=7.4 \mathrm{~Hz}, 3 \mathrm{H}, 10\right), 0.46$ (dd, $\left.J=4.0,1.0 \mathrm{~Hz}, 1 \mathrm{H}, 7^{\prime \prime}\right)$.

${ }^{13} \mathrm{C}$ NMR $\left(\mathrm{CDCl}_{3}, 101 \mathrm{MHz} ; \delta \mathrm{ppm}\right)$ :

$141.22(\mathrm{CH}, \mathbf{6}), 110.17(\mathrm{CH}, \mathbf{5}), 67.76\left(\mathrm{CH}_{2}, \mathbf{2}\right), 28.44(\mathrm{C}, 4), 25.84\left(\mathrm{CH}_{2}, \mathbf{9}\right), 24.67\left(\mathrm{CH}_{2}, 7\right), 22.33(\mathrm{C}, 3), 15.33\left(\mathrm{CH}_{3}, \mathbf{8}\right), 11.94$ $\left(\mathrm{CH}_{3}, \mathbf{1 0}\right)$.

HRMS (EI) m/z: $[\mathrm{M}+\mathrm{H}]^{+}$Calcd for $\mathrm{C}_{9} \mathrm{H}_{14} \mathrm{O}$ 139.1123; Found: 139.1119

1-methyl-6-propyl-3-oxabicyclo[4.1.0]hept-4-ene [2i]

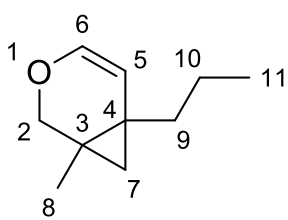

\author{
Formula : $\mathrm{C}_{10} \mathrm{H}_{16} \mathrm{O}$ \\ $\mathrm{M}: \mathbf{1 5 2 . 2 4}$ g.mol ${ }^{-1}$ \\ Aspect : Colorless oil \\ $\mathrm{R}_{\mathrm{f}}=0.72$ (petroleum ether/ $\mathrm{Et}_{2} \mathrm{O}, 98 / 2 ;$ Kagi-Mosher)
}

According to the experimental procedure (2) and starting from $811 \mathrm{mg}(5.3 \mathrm{mmol}, 1 \mathrm{eq} ., \mathrm{M}=152.24) \mathrm{of}$ [1i], $3 \mathrm{mg}(5 \mu \mathrm{mol}, 0.1$ mol\%) of IPrAuCl and $5 \mathrm{mg}(5 \mu \mathrm{mol}, 0.1 \mathrm{~mol} \%)$ of $\mathbf{N a B A r}^{\mathbf{F}}$ in Toluene (0.5M) the 1-methyl-6-propyl-3oxabicyclo[4.1.0]hept-4-ene [2i] was obtained after $15 \mathrm{~h}$ of reaction as a colorless oil $(688 \mathrm{mg}, 85 \%)$.

${ }^{1} \mathrm{H}$ NMR $\left(\mathrm{CDCl}_{3}, 400 \mathrm{MHz} ; \delta\right.$ ppm) :

$6.11(\mathrm{~d}, J=5.8 \mathrm{~Hz}, 1 \mathrm{H}, \mathbf{6}), 5.06(\mathrm{~d}, J=5.9 \mathrm{~Hz}, 1 \mathrm{H}, \mathbf{5}), 3.91\left(\mathrm{~d}, J=10.2 \mathrm{~Hz}, 1 \mathrm{H}, \mathbf{2}^{\prime \prime}\right), 3.46\left(\mathrm{~d}, J=10.2 \mathrm{~Hz}, 1 \mathrm{H}, \mathbf{2}^{\prime}\right), 1.51-1.38(\mathrm{~m}$, $\left.4 \mathrm{H}, 9^{\prime \prime}, 10\right), 1.32-1.26\left(\mathrm{~m}, 1 \mathrm{H}, 9^{\prime}\right), 1.12(\mathrm{~s}, 3 \mathrm{H}, \mathbf{8}), 1.06\left(\mathrm{~d}, J=4.0 \mathrm{~Hz}, 1 \mathrm{H}, 7^{\prime}\right), 0.91(\mathrm{t}, J=7.2 \mathrm{~Hz}, 2 \mathrm{H}, 11), 0.46(\mathrm{dd}, J=4.0,1.0$ $\left.\mathrm{Hz}, 1 \mathrm{H}, 7^{\prime \prime}\right)$.

${ }^{13} \mathrm{C}$ NMR $\left(\mathrm{CDCl}_{3}, 101 \mathrm{MHz} ; \delta\right.$ ppm) :

$140.90(\mathrm{CH}, \mathbf{6}), 110.46(\mathrm{CH}, \mathbf{5}), 67.62\left(\mathrm{CH}_{2}, \mathbf{2}\right), 35.06\left(\mathrm{CH}_{2}, \mathbf{9}\right), 28.33(\mathrm{C}, 4), 24.88\left(\mathrm{CH}_{2}, 7\right), 21.28(\mathrm{C}, 3), 20.85\left(\mathrm{CH}_{2}, 10\right), 15.55$ $\left(\mathrm{CH}_{3}, \mathbf{8}\right), 14.35\left(\mathrm{CH}_{3}, \mathbf{1 1}\right)$.

HRMS (EI) m/z: $[\mathrm{M}+\mathrm{H}]^{+}$Calcd for $\mathrm{C}_{10} \mathrm{H}_{16} \mathrm{O}$ 153.1279; Found: 153.1273 


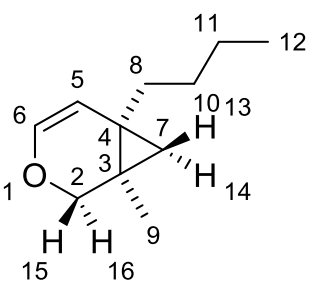

Formula : $\mathrm{C}_{11} \mathrm{H}_{18} \mathrm{O}$

$\mathrm{M}: 166.26$ g.mol ${ }^{-1}$

Aspect : Light yellow oil

$\mathrm{R}_{\mathrm{f}}=0.59$ (petroleum ether/Et $\mathrm{O}_{2} \mathrm{O}, 90 / 10 ;$ Kagi-Mosher)

According to the experimental procedure (2) and starting from $607 \mathrm{mg}$ (3.7 mmol, 1 eq., $\mathrm{M}=166.26)$ of [1j], $12 \mathrm{mg}(20 \mu \mathrm{mol}, 0.5$ mol\%) of IPrAuCl and $18 \mathrm{mg}(20 \mu \mathrm{mol}, 0.5 \mathrm{~mol} \%)$ of NaBAr ${ }^{\mathbf{F}}$, in Toluene (0.5M) the 6-butyl-1-methyl-3oxabicyclo[4.1.0]hept-4-ene [2j] was obtained after $21 \mathrm{~h}$ of reaction as a light yellow oil (552 $\mathrm{mg}, 91 \%)$.

${ }^{1} \mathrm{H}$ NMR $\left(\mathrm{CDCl}_{3}, 400 \mathrm{MHz} ; \delta\right.$ ppm) :

$6.11(\mathrm{~d}, J=5.8 \mathrm{~Hz}, 1 \mathrm{H}, \mathbf{6}), 5.06(\mathrm{~d}, J=5.9 \mathrm{~Hz}, 1 \mathrm{H}, \mathbf{5}), 3.91(\mathrm{~d}, J=10.2 \mathrm{~Hz}, 1 \mathrm{H}, \mathbf{1 5}), 3.46(\mathrm{~d}, J=10.2 \mathrm{~Hz}, 1 \mathrm{H}, \mathbf{1 6}), 1.53-1.45$ $\left(\mathrm{m}, 1 \mathrm{H}, \mathbf{8}^{\prime}\right), 1.45-1.26\left(\mathrm{~m}, 5 \mathrm{H}, \mathbf{8}^{\prime \prime}, \mathbf{1 0}, \mathbf{1 1}\right), 1.13(\mathrm{~s}, 3 \mathrm{H}, \mathbf{9}), 1.06(\mathrm{~d}, J=4.0 \mathrm{~Hz}, 1 \mathrm{H}, 13), 0.91(\mathrm{t}, J=7.1 \mathrm{~Hz}, 3 \mathrm{H}, 12), 0.46(\mathrm{dd}, J=$ 4.0, $1.0 \mathrm{~Hz}, 1 \mathrm{H}, 14)$.

${ }^{13} \mathrm{C}$ NMR $\left(\mathrm{CDCl}_{3}, 101 \mathrm{MHz} ; \delta\right.$ ppm) :

$140.93(\mathrm{CH}, \mathbf{6}), 110.54(\mathrm{CH}, \mathbf{5}), 67.65\left(\mathrm{CH}_{2}, \mathbf{2}\right), 32.64\left(\mathrm{CH}_{2}, \mathbf{8}\right), 29.99\left(\mathrm{CH}_{2}, 7\right), 28.34(\mathrm{C}, \mathbf{3}), 24.86\left(\mathrm{CH}_{2}, 11\right), 22.99\left(\mathrm{CH}_{2}, 10\right)$, $21.33(\mathrm{C}, 4), 15.51\left(\mathrm{CH}_{3}, 9\right), 14.14\left(\mathrm{CH}_{3}, \mathbf{1 2}\right)$.

HRMS (EI) m/z : [M+H] $]^{+}$Calcd for $\mathrm{C}_{11} \mathrm{H}_{18} \mathrm{O}$ 167.1436; Found 167.1429

1-methyl-6-pentyl-3-oxabicyclo[4.1.0]hept-4-ene [2k]

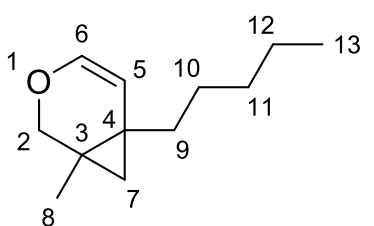

\author{
Formula : $\mathrm{C}_{14} \mathrm{H}_{24} \mathrm{O}_{2}$ \\ $\mathrm{M}: 180.29$ g. $\mathrm{mol}^{-1}$ \\ Aspect : Colorless oil \\ $\mathrm{R}_{\mathrm{f}}=0.82$ (Pentane $/ \mathrm{Et}_{2} \mathrm{O}, 96 / 4$; Kagi-Mosher)
}

According to the experimental procedure (2) and starting from $1 \mathrm{~g}(5.6 \mathrm{mmol}, 1$ eq., $\mathrm{M}=180.29)$ of [1k], $7 \mathrm{mg}(11 \mu \mathrm{mol}, 0.2$ mol\%) of IPrAuCl and $10 \mathrm{mg}$ (11 $\mu \mathrm{mol}, 0.2 \mathrm{~mol} \%)$ of NaBAr ${ }^{\mathbf{F}}$, in Toluene (0.5M) the 1-methyl-6-pentyl-3oxabicyclo[4.1.0]hept-4-ene [2k] was obtained after $6.5 \mathrm{~h}$ of reaction as an Colorless oil (780 $\mathrm{mg}, 78 \%$ ).

${ }^{1} \mathrm{H}$ NMR $\left(\mathrm{CDCl}_{3}, 400 \mathrm{MHz} ; \delta\right.$ ppm) :

$6.11(\mathrm{~d}, J=5.9 \mathrm{~Hz}, 1 \mathrm{H}, \mathbf{6}), 5.06(\mathrm{~d}, J=5.8 \mathrm{~Hz}, 1 \mathrm{H}, \mathbf{5}), 3.91\left(\mathrm{~d}, J=10.2 \mathrm{~Hz}, 1 \mathrm{H}, \mathbf{2}^{\prime \prime}\right), 3.55-3.35\left(\mathrm{~m}, 1 \mathrm{H}, 2^{\prime}\right), 1.53-1.21(\mathrm{~m}, 7 \mathrm{H}$, 9, 10, 11, 12), $1.13(\mathrm{~s}, 3 \mathrm{H}, 8), 1.06\left(\mathrm{~d}, J=4.0 \mathrm{~Hz}, 1 \mathrm{H}, 7^{\prime}\right), 0.90(\mathrm{t}, J=7.0 \mathrm{~Hz}, 3 \mathrm{H}, 13), 0.46\left(\mathrm{dd}, J=4.0,1.3 \mathrm{~Hz}, 1 \mathrm{H}, 7^{\prime \prime}\right)$.

${ }^{13} \mathrm{C}$ NMR $\left(\mathrm{CDCl}_{3}, 101 \mathrm{MHz} ; \delta \mathrm{ppm}\right)$ :

$140.91(\mathrm{CH}, 6), 110.52(\mathrm{CH}, 5), 67.63\left(\mathrm{CH}_{2}, 2\right), 32.89\left(\mathrm{CH}_{2}, 9\right), 32.13\left(\mathrm{CH}_{2}, 11\right), 28.32(\mathrm{C}, 4), 27.40\left(\mathrm{CH}_{2}, 7\right), 24.85\left(\mathrm{CH}_{2}, 10\right), 22.66$ $\left(\mathrm{CH}_{2}, 12\right), 21.35(\mathrm{C}, 3), 15.49\left(\mathrm{CH}_{3}, 8\right), 14.06\left(\mathrm{CH}_{3}, 13\right)$.

HRMS (EI) m/z : $[\mathrm{M}+\mathrm{H}]^{+}$Calcd for $\mathrm{C}_{12} \mathrm{H}_{20} \mathrm{O}$ 181.1592; Found 181.1585

6-heptyl-1-methyl-3-oxabicyclo[4.1.0]hept-4-ene [21]

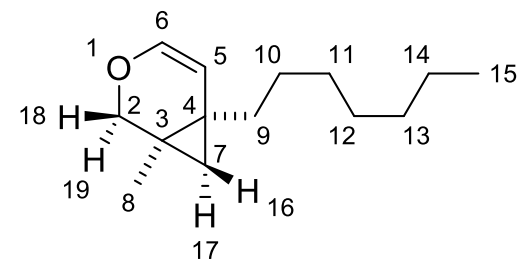

\author{
Formula : $\mathrm{C}_{14} \mathrm{H}_{24} \mathrm{O}_{2}$ \\ $\mathrm{M}: 208.35$ g. $\mathrm{mol}^{-1}$ \\ Aspect : Colorless oil \\ $\mathrm{R}_{\mathrm{f}}=0.72$ (Pentane $/ \mathrm{Et}_{2} \mathrm{O}, 98 / 2 ;$ Kagi-Mosher)
}

According to the experimental procedure (2) and starting from $1.5 \mathrm{~g}(7.2 \mathrm{mmol}, 1 \mathrm{eq} ., \mathrm{M}=208.35)$ of [11], $5 \mathrm{mg}(7 \mu \mathrm{mol}, 0.1$ mol\%) of IPrAuCl and $6 \mathrm{mg}(7 \mu \mathrm{mol}, 0.1 \mathrm{~mol} \%)$ of $\mathbf{N a B A r}{ }^{\mathrm{F}}$, in Toluene $(0.5 \mathrm{M})$ the 6-heptyl-1-methyl-3oxabicyclo[4.1.0]hept-4-ene [21] was obtained after 15h of reaction as an Colorless oil (957 mg, yield: $76 \%$, conv.: 84\%). 
${ }^{1} \mathrm{H}$ NMR $\left(\mathrm{CDCl}_{3}, 400 \mathrm{MHz} ; \delta\right.$ ppm) :

$6.11(\mathrm{~d}, J=5.8 \mathrm{~Hz}, 1 \mathrm{H}, \mathbf{6}), 5.06(\mathrm{~d}, J=5.8 \mathrm{~Hz}, 1 \mathrm{H}, \mathbf{5}), 3.91(\mathrm{~d}, J=10.2 \mathrm{~Hz}, 1 \mathrm{H}, \mathbf{1 9}), 3.46(\mathrm{dd}, J=10.2,1.3 \mathrm{~Hz}, 1 \mathrm{H}, \mathbf{1 8}), 1.57-$ $1.21(\mathrm{~m}, 12 \mathrm{H}, \mathbf{9}, \mathbf{1 0}, \mathbf{1 1}, \mathbf{1 2}, \mathbf{1 3}, \mathbf{1 4}), 1.12(\mathrm{~s}, 3 \mathrm{H}, \mathbf{8}), 1.06(\mathrm{~d}, J=4.0 \mathrm{~Hz}, 1 \mathrm{H}, 17), 0.92-0.85(\mathrm{~m}, 3 \mathrm{H}, 15), 0.46(\mathrm{dd}, J=4.0,1.3$ $\mathrm{Hz}, 1 \mathrm{H}, \mathbf{1 6})$.

${ }^{13} \mathrm{C}$ NMR $\left(\mathrm{CDCl}_{3}, 101 \mathrm{MHz} ; \delta\right.$ ppm) :

$140.90(\mathrm{CH}, \mathbf{6}), 110.53(\mathrm{CH}, \mathbf{5}), 67.63\left(\mathrm{CH}_{2}, \mathbf{2}\right), 32.94\left(\mathrm{CH}_{2}, \mathbf{9}\right), 31.85\left(\mathrm{CH}_{2}, \mathbf{1 3}\right), 29.89\left(\mathrm{CH}_{2}, \mathbf{1 2}\right), 29.32\left(\mathrm{CH}_{2}, 11\right), 28.32(\mathrm{C}, 4)$, $27.74\left(\mathrm{CH}_{2}, \mathbf{1 0}\right), 24.85\left(\mathrm{CH}_{2}, 7\right), 22.66\left(\mathrm{CH}_{2}, \mathbf{1 4}\right), 21.35(\mathrm{C}, \mathbf{3}), 15.50\left(\mathrm{CH}_{3}, \mathbf{8}\right), 14.09\left(\mathrm{CH}_{3}, \mathbf{1 5}\right)$.

HRMS (EI) m/z : [M+H $]^{+}$Calcd for $\mathrm{C}_{14} \mathrm{H}_{24} \mathrm{O} 209.1905$; Found 209.1897

(1-methyl-3-oxabicyclo[4.1.0]hept-4-en-6-yl)methanol [2m]

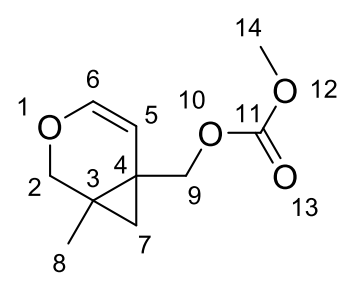

\author{
Formula : $\mathrm{C}_{10} \mathrm{H}_{14} \mathrm{O}_{4}$ \\ $\mathrm{M}: 198.22 \mathrm{~g} \cdot \mathrm{mol}^{-1}$ \\ Aspect : Colorless oil \\ $\mathrm{R}_{\mathrm{f}}=0.26$ (petroleum ether $/ \mathrm{Et}_{2} \mathrm{O}, 50 / 50 ;$ Kagi-Mosher)
}

According to the experimental procedure (2) and starting from $112 \mathrm{mg}(0.57 \mathrm{mmol}, 1 \mathrm{eq}$.) of carbonate [1 m], $4 \mathrm{mg}(6 \mu \mathrm{mol}, 1$ mol\%) of IPrAuCl and $5 \mathrm{mg}(6 \mu \mathrm{mol}, 1 \mathrm{~mol} \%)$ of $\mathbf{N a B A r}{ }^{\mathbf{F}}$, in Toluene $(0.5 \mathrm{M})$ and after $3 \mathrm{~h}$ of reaction, (1-methyl-3oxabicyclo[4.1.0]hept-4-en-6-yl)methanol [2m] was obtained as an Colorless oil (57 mg, $51 \%)$.

${ }^{1} \mathrm{H}$ NMR $\left(\mathrm{CDCl}_{3}, 400 \mathrm{MHz} ; \delta\right.$ ppm) :

$6.16(\mathrm{~d}, J=5.8 \mathrm{~Hz}, 1 \mathrm{H}, \mathbf{6}), 5.19(\mathrm{~d}, J=5.8 \mathrm{~Hz}, 1 \mathrm{H}, \mathbf{5}), 4.41\left(\mathrm{dd}, J=11.5,0.9 \mathrm{~Hz}, 1 \mathrm{H}, \mathbf{2}^{\prime \prime}\right), 4.01\left(\mathrm{~d}, J=11.5 \mathrm{~Hz}, 1 \mathrm{H}, \mathbf{2}^{\prime}\right), 3.94(\mathrm{~d}, J$ $\left.=10.3 \mathrm{~Hz}, 1 \mathrm{H}, \mathbf{9}^{\prime \prime}\right), 3.79(\mathrm{~s}, 3 \mathrm{H}, \mathbf{1 4}), 3.55-3.48\left(\mathrm{~m}, 1 \mathrm{H}, \mathbf{9}^{\prime}\right), 1.26\left(\mathrm{~d}, J=4.6 \mathrm{~Hz}, 1 \mathrm{H}, 7^{\prime}\right), 1.20(\mathrm{~s}, 3 \mathrm{H}, \mathbf{8}), 0.80(\mathrm{dd}, J=4.6,1.3 \mathrm{~Hz}$, $\left.1 \mathrm{H}, 7^{\prime \prime}\right)$.

${ }^{13} \mathrm{C}$ NMR $\left(\mathrm{CDCl}_{3}, 101 \mathrm{MHz} ; \delta\right.$ ppm) :

$155.92(\mathrm{C}, \mathbf{1 1}), 142.06(\mathrm{CH}, \mathbf{6}), 107.70(\mathrm{CH}, \mathbf{5}), 70.95\left(\mathrm{CH}_{2}, \mathbf{2}\right), 66.81\left(\mathrm{CH}_{2}, \mathbf{9}\right), 54.75\left(\mathrm{CH}_{3}, \mathbf{1 4}\right), 29.25(\mathrm{C}, 4), 23.85\left(\mathrm{CH}_{2}, 7\right)$, $20.89(\mathrm{C}, \mathbf{3}), 15.44\left(\mathrm{CH}_{3}, \mathbf{8}\right)$.

HRMS (EI) m/z : [M+H] $]^{+}$Calcd for $\mathrm{C}_{10} \mathrm{H}_{14} \mathrm{O}_{4}$ 199.0970; Found 199.0963

\title{
Synthesis of [2n]
}
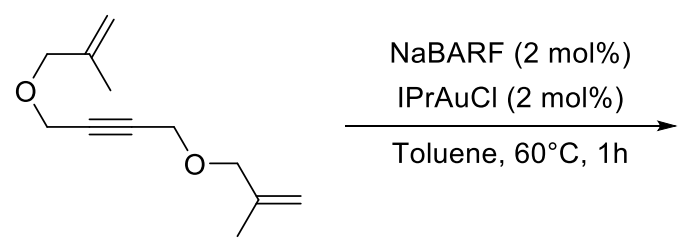

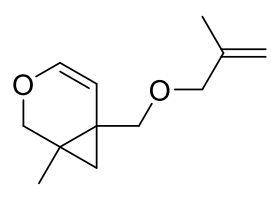

$44 \%$

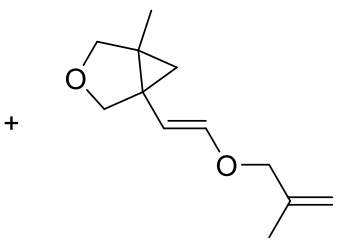

$15 \%$

According to the experimental procedure (2) and starting from $270 \mathrm{mg}$ (1.4 mmol, 1 eq.) of 1,4-bis((2-methylallyl)oxy)but-2yne, $19 \mathrm{mg}(30 \mu \mathrm{mol}, 2 \mathrm{~mol} \%)$ of IPrAuCl and $27 \mathrm{mg}(30 \mu \mathrm{mol}, 2 \mathrm{~mol} \%)$ of NaBAr , in Toluene $(0.5 \mathrm{M})$ and after $1 \mathrm{~h}$ of reaction, the 1-methyl-6-((2-methylallyl)oxy)methyl)-3-oxabicyclo[4.1.0]hept-4-ene [2n] was obtained as an Colorless oil (127 mg, $44 \%)$ and the (E)-1-methyl-5-(2-((2-methylallyl)oxy)vinyl)-3-oxabicyclo[3.1.0]hexane [2n'] was obtained as an Colorless oil (17 mg, $15 \%)$.

1-methyl-6-(((2-methylallyl)oxy)methyl)-3-oxabicyclo[4.1.0]hept-4-ene [2n]

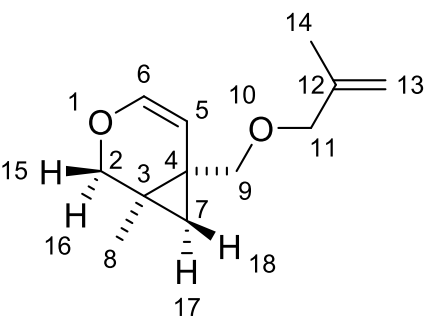

\author{
Formula : $\mathrm{C}_{12} \mathrm{H}_{18} \mathrm{O}_{2}$ \\ M : 194.27 g.mol ${ }^{-1}$ \\ Aspect : Colorless oil \\ $\mathbf{R}_{\mathbf{f}}=0.42$ (petroleum ether/Et $2 \mathrm{O}, 98 / 2 ;$ Kagi-Mosher)
}


${ }^{1} \mathrm{H}$ NMR $\left(\mathrm{CDCl}_{3}, 400 \mathrm{MHz} ; \delta\right.$ ppm) :

$6.16(\mathrm{~d}, J=5.8 \mathrm{~Hz}, 1 \mathrm{H}, \mathbf{6}), 5.24(\mathrm{~d}, J=5.8 \mathrm{~Hz}, 1 \mathrm{H}, \mathbf{5}), 4.96(\mathrm{dq}, J=2.3,1.1 \mathrm{~Hz}, 1 \mathrm{H}, 13 \mathrm{\prime}), 4.93-4.87$ (m, $\left.1 \mathrm{H}, 13{ }^{\prime \prime}\right), 3.98-3.89$

$\left(\mathrm{m}, 2 \mathrm{H}, 9^{\prime \prime}\right), 3.88(\mathrm{~d}, J=12.6 \mathrm{~Hz}, 1 \mathrm{H}, \mathbf{1 1}), 3.56-3.48\left(\mathrm{~m}, 2 \mathrm{H}, 9^{\prime}, \mathbf{1 5}\right), 3.30(\mathrm{~d}, J=10.2 \mathrm{~Hz}, 1 \mathrm{H}, \mathbf{1 6}), 1.75(\mathrm{t}, J=1.2 \mathrm{~Hz}, 3 \mathrm{H}, \mathbf{1 4})$, $1.18(\mathrm{~s}, 3 \mathrm{H}, \mathbf{8}), 1.28-1.12(\mathrm{~m}, 1 \mathrm{H}, \mathbf{1 8}), 0.68(\mathrm{dd}, J=4.3,1.3 \mathrm{~Hz}, 1 \mathrm{H}, 17)$.

${ }^{13} \mathrm{C}$ NMR $\left(\mathrm{CDCl}_{3}, 101 \mathrm{MHz} ; \delta\right.$ ppm) :

142.30 (C, 12), $141.49(\mathrm{CH}, \mathbf{6}), 112.41\left(\mathrm{CH}_{2}, \mathbf{1 3}\right), 108.95(\mathrm{CH}, \mathbf{5}), 74.90\left(\mathrm{CH}_{2}, \mathbf{1 1}\right), 72.18\left(\mathrm{CH}_{2}, \mathbf{2}\right), 67.09\left(\mathrm{CH}_{2}, 9\right), 28.71(\mathrm{C}, \mathbf{3})$, $23.38\left(\mathrm{CH}_{2}, 7\right), 21.64(\mathrm{C}, 4), 19.44\left(\mathrm{CH}_{3}, 14\right), 15.32\left(\mathrm{CH}_{3}, 8\right)$.

HRMS (EI) m/z : [M+H] $]^{+}$Calcd for $\mathrm{C}_{12} \mathrm{H}_{18} \mathrm{O}_{2}$ 195.1385; Found 195.1379

(E)-1-methyl-5-(2-((2-methylallyl)oxy)vinyl)-3-oxabicyclo[3.1.0]hexane [2n']

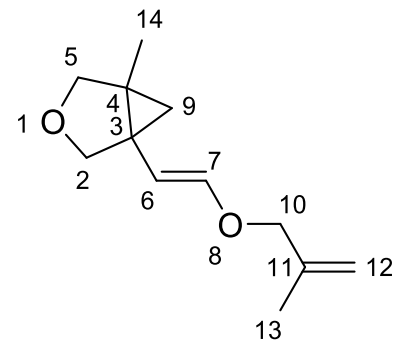

Formula : $\mathrm{C}_{12} \mathrm{H}_{18} \mathrm{O}_{2}$

M : 194.27 g.mol ${ }^{-1}$

Aspect : Colorless oil

$\mathbf{R}_{\mathbf{f}}=0.17$ (petroleum ether $/ \mathrm{Et}_{2} \mathrm{O}, 98 / 2 ;$ Kagi-Mosher)

${ }^{1} \mathrm{H}$ NMR $\left(\mathrm{CDCl}_{3}, 400 \mathrm{MHz} ; \delta\right.$ ppm) :

$6.23(\mathrm{~d}, J=12.6 \mathrm{~Hz}, 1 \mathrm{H}, 7), 5.02-4.91(\mathrm{~m}, 2 \mathrm{H}, 12), 4.86(\mathrm{~d}, J=12.6 \mathrm{~Hz}, 1 \mathrm{H}, 6), 4.12(\mathrm{~s}, 2 \mathrm{H}, 10), 3.82\left(\mathrm{~d}, J=8.1 \mathrm{~Hz}, 1 \mathrm{H}, 2 \mathbf{2}^{\prime}\right), 3.76(\mathrm{~d}$, $\left.J=8.1 \mathrm{~Hz}, 1 \mathrm{H}, \mathbf{5}^{\prime \prime}\right), 3.62\left(\mathrm{dd}, J=8.1,1.2 \mathrm{~Hz}, 1 \mathrm{H}, \mathbf{2}^{\prime \prime}\right), 3.53\left(\mathrm{~d}, J=8.0 \mathrm{~Hz}, 1 \mathrm{H}, \mathbf{5}^{\prime}\right), 1.78-1.73(\mathrm{~m}, 3 \mathrm{H}, 13), 1.08(\mathrm{~s}, 3 \mathrm{H}, 14), 0.75(\mathrm{~d}, J=$ $\left.4.6 \mathrm{~Hz}, 1 \mathrm{H}, 9^{\prime}\right), 0.52\left(\mathrm{~d}, J=4.5 \mathrm{~Hz}, 1 \mathrm{H}, 9^{\prime \prime}\right)$.

${ }^{13} \mathrm{C}$ NMR $\left(\mathrm{CDCl}_{3}, 101 \mathrm{MHz} ; \delta\right.$ ppm) :

$148.18(\mathrm{CH}, 7), 141.04(\mathrm{C}, 11), 112.71\left(\mathrm{CH}_{2}, 12\right), 101.21(\mathrm{CH}, 6), 74.71\left(\mathrm{CH}_{2}, 10\right), 73.55\left(\mathrm{CH}_{2}, 2\right), 73.55\left(\mathrm{CH}_{2}, 5\right), 30.63(\mathrm{C}, 3), 28.71$ $(\mathrm{C}, 4), 19.32\left(\mathrm{CH}_{3}, 13\right), 17.51\left(\mathrm{CH}_{2}, 9\right), 13.29\left(\mathrm{CH}_{3}, 14\right)$.

HRMS (EI) m/z : [M+H] $]^{+}$Calcd for $\mathrm{C}_{12} \mathrm{H}_{18} \mathrm{O}_{2}$ 195.1385; Found 195.1378

tert-butyl 2-((1-methyl-3-oxabicyclo[4.1.0]hept-4-en-6-yl)methoxy)acetate [2o]

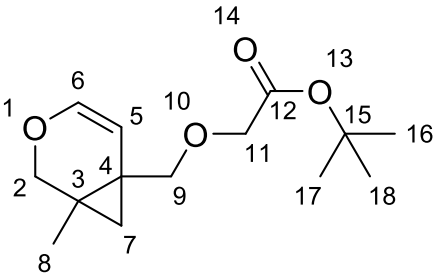

\author{
Formula : $\mathrm{C}_{14} \mathrm{H}_{22} \mathrm{O}_{4}$ \\ $\mathrm{M}: 254.33 \mathrm{~g} \cdot \mathrm{mol}^{-1}$ \\ Aspect : Colorless oil \\ $\mathrm{R}_{\mathrm{f}}=0.71$ (petroleum ether $/ \mathrm{Et}_{2} \mathrm{O}, 70 / 30$; Kagi-Mosher)
}

According to the experimental procedure (2) and starting from $156 \mathrm{mg}(0.61 \mathrm{mmol}, 1$ eq.) of ethyl 2-((4-((2-methylallyl)oxy)but2-yn-1-yl)oxy)acetate, $4 \mathrm{mg}(4 \mu \mathrm{mol}, 1 \mathrm{~mol} \%)$ of IPrAuCl and $5 \mathrm{mg}(6 \mu \mathrm{mol}, 1 \mathrm{~mol} \%)$ of NaBAr ${ }^{\mathbf{F}}$, in Toluene $(0.5 \mathrm{M})$ and after $9 \mathrm{~h}$ of reaction, the tert-butyl 2-((1-methyl-3-oxabicyclo[4.1.0]hept-4-en-6-yl)methoxy)acetate [2o] was obtained as a colorless oil (68 $\mathrm{mg}, 44 \%)$.

${ }^{1} \mathrm{H}$ NMR $\left(\mathrm{CDCl}_{3}, 400 \mathrm{MHz} ; \boldsymbol{\delta} \mathbf{~ p p m}\right)$ :

$6.17(\mathrm{~d}, J=5.8 \mathrm{~Hz}, 1 \mathrm{H}, \mathbf{6}), 5.33(\mathrm{~d}, J=5.8 \mathrm{~Hz}, 1 \mathrm{H}, \mathbf{5}), 3.98(\mathrm{~s}, 2 \mathrm{H}, \mathbf{1 1}), 3.94\left(\mathrm{~d}, J=10.3 \mathrm{~Hz}, 1 \mathrm{H}, \mathbf{9}^{\prime \prime}\right), 3.69$ (dd, $J=9.9,0.7 \mathrm{~Hz}$, $\left.1 \mathrm{H}, \mathbf{2}^{\prime \prime}\right), 3.52$ (dt, $\left.J=10.4,1.4,0.7 \mathrm{~Hz}, 1 \mathrm{H}, 9^{\prime}\right), 3.48$ (d, $\left.J=9.9 \mathrm{~Hz}, 1 \mathrm{H}, 2^{\prime}\right), 1.48$ (s, 9H, 16, 17, 18), 1.21 (d, $\left.J=4.5 \mathrm{~Hz}, 1 \mathrm{H}, 7^{\prime}\right)$, $1.21(\mathrm{~s}, 3 \mathrm{H}, \mathbf{8}), 0.70$ (dd, $\left.J=4.5,1.3 \mathrm{~Hz}, 1 \mathrm{H}, 7^{\prime \prime}\right)$.

${ }^{13} \mathrm{C}$ NMR $\left(\mathrm{CDCl}_{3}, 101 \mathrm{MHz} ; \delta\right.$ ppm) :

$170.26(\mathrm{C}=\mathrm{O}, \mathbf{1 2}), 142.11(\mathrm{CH}, \mathbf{6}), 109.19(\mathrm{CH}, \mathbf{5}), 81.99(\mathrm{C}, \mathbf{1 5}), 74.29\left(\mathrm{CH}_{2}, \mathbf{9}\right), 68.88\left(\mathrm{CH}_{2}, \mathbf{1 1}\right), 67.51\left(\mathrm{CH}_{2}, 2\right), 29.39(\mathrm{C}, 4)$, $28.62\left(3 \mathrm{xCH}_{3}, \mathbf{1 6}, \mathbf{1 7}, \mathbf{1 8}\right), 23.81\left(\mathrm{CH}_{2}, 7\right), 22.10(\mathrm{C}, \mathbf{3}), 15.85\left(\mathrm{CH}_{3}, \mathbf{8}\right)$.

HRMS (EI) m/z : [M+H] $]^{+}$Calcd for $\mathrm{C}_{14} \mathrm{H}_{22} \mathrm{O}_{4}$ 255.1596; Found 255.1587 


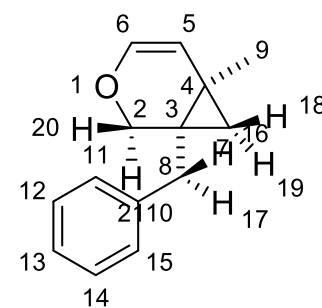

14

\author{
Formula : $\mathrm{C}_{14} \mathrm{H}_{16} \mathrm{O}$ \\ $\mathrm{M}: 200.28{\text { g. } \mathrm{mol}^{-1}}^{-1}$ \\ Aspect : Colorless oil \\ $\mathrm{R}_{\mathrm{f}}=0.48$ (petroleum ether/Et ${ }_{2} \mathrm{O}, 98 / 2$; Kagi-Mosher)
}

According to the experimental procedure (2) and starting from $305 \mathrm{mg}$ (1.5 mmol, 1 eq.) of [1p], $19 \mathrm{mg}(30 \mu \mathrm{mol}, 2 \mathrm{~mol} \%)$ of IPrAuCl and $27 \mathrm{mg}(30 \mu \mathrm{mol}, 2 \mathrm{~mol} \%)$ of $\mathbf{N a B A r}{ }^{\mathbf{F}}$, in Toluene $(0.5 \mathrm{M})$ and after $3 \mathrm{~h}$ of reaction, the 1-benzyl-6-methyl-3oxabicyclo[4.1.0]hept-4-ene [2p] was obtained as a colorless oil (286 mg, $94 \%$ ).

${ }^{1} \mathrm{H}$ NMR $\left(\mathrm{CDCl}_{3}, 400 \mathrm{MHz} ; \delta\right.$ ppm) :

$7.34-7.25(\mathrm{~m}, 2 \mathrm{H}, \mathbf{1 1}, \mathbf{1 5}), 7.29-7.14(\mathrm{~m}, 3 \mathrm{H}, \mathbf{1 2}, \mathbf{1 3}, \mathbf{1 4}), 6.10(\mathrm{~d}, \mathrm{~J}=5.7 \mathrm{~Hz}, 1 \mathrm{H}, \mathbf{6}), 5.07(\mathrm{~d}, \mathrm{~J}=5.8 \mathrm{~Hz}, 1 \mathrm{H}, \mathbf{5}), 3.80(\mathrm{~d}, \mathrm{~J}=$ $10.4 \mathrm{~Hz}, 1 \mathrm{H}, \mathbf{2 0}), 3.54-3.46(\mathrm{~m}, 1 \mathrm{H}, \mathbf{2 1}), 2.88(\mathrm{~d}, \mathrm{~J}=15.1 \mathrm{~Hz}, 1 \mathrm{H}, \mathbf{1 6}), 2.75(\mathrm{~d}, \mathrm{~J}=15.2 \mathrm{~Hz}, 1 \mathrm{H}, 17), 1.30$ (s, 3H, 9), 1.20 (d, J = $4.2 \mathrm{~Hz}, 1 \mathrm{H}, \mathbf{1 8}), 0.77(\mathrm{dd}, \mathrm{J}=4.3,1.3 \mathrm{~Hz}, 1 \mathrm{H}, \mathbf{1 9})$.

${ }^{13} \mathrm{C}$ NMR $\left(\mathrm{CDCl}_{3}, 101 \mathrm{MHz} ; \delta\right.$ ppm) :

$140.85(\mathrm{CH}, \mathbf{6}), 139.22(\mathrm{C}, \mathbf{1 0}), 128.67(2 \times \mathrm{xH}, \mathbf{1 1}, \mathbf{1 5}), 128.39(2 \times \mathrm{xH}, \mathbf{1 2}, \mathbf{1 4}), 126.21(\mathrm{CH}, \mathbf{1 3}), 112.34(\mathrm{CH}, \mathbf{5}), 65.95(\mathrm{CH} 2, \mathbf{2})$, $36.75\left(\mathrm{CH}_{2}, \mathbf{8}\right), 32.10(\mathrm{C}, \mathbf{3}), 25.15\left(\mathrm{CH}_{2}, 7\right), 19.32\left(\mathrm{CH}_{3}, 9\right), 16.87(\mathrm{C}, 4)$.

HRMS (EI) $\mathbf{m} / \mathbf{z}:[\mathrm{M}+\mathrm{H}]^{+}$Calcd for $\mathrm{C}_{14} \mathrm{H}_{16} \mathrm{O} 201.1279$; Found 201.1273

1-methyl-6-(p-tolyl)-3-oxabicyclo[4.1.0]hept-4-ene [2q]

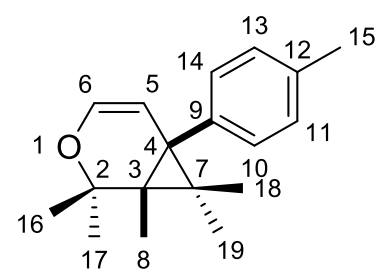

\author{
Formula : $\mathrm{C}_{14} \mathrm{H}_{16} \mathrm{O}$ \\ $\mathrm{M}: 200.28 \mathrm{~g} \cdot \mathrm{mol}^{-1}$ \\ Aspect : Colorless oil \\ $\mathrm{R}_{\mathrm{f}}=0.47$ (Pentane ; Kagi-Mosher)
}

According to the experimental procedure (2) and starting from $143 \mathrm{mg}(0.7 \mathrm{mmol}, 1 \mathrm{eq}$.$) of [1q], 9 \mathrm{mg}(14 \mu \mathrm{mol}, 2 \mathrm{~mol} \%)$ of IPrAuCl and $13 \mathrm{mg}(14 \mu \mathrm{mol}, 2 \mathrm{~mol} \%)$ of $\mathbf{N a B A r}{ }^{\mathbf{F}}$, in Toluene $(0.5 \mathrm{M})$ and after $3 \mathrm{~h}$ of reaction, the 1-methyl-6-(p-tolyl)-3oxabicyclo[4.1.0]hept-4-ene [2q] was obtained as a colorless oil (93 $\mathrm{mg}, 65 \%)$.

${ }^{1} \mathrm{H}$ NMR $\left(\mathrm{CDCl}_{3}, 400 \mathrm{MHz} ; \delta\right.$ ppm) :

$7.14(\mathrm{~d}, \mathrm{~J}=8.3 \mathrm{~Hz}, 2 \mathrm{H}, \mathbf{1 0}, \mathbf{1 4}), 7.11(\mathrm{~d}, \mathrm{~J}=8.3 \mathrm{~Hz}, 2 \mathrm{H}, \mathbf{1 1}, \mathbf{1 3}), 6.20$ (d, J = 5.8 Hz, 1H, 6), 5.19 (d, J = 5.8 Hz, 1H, 5), 4.06 (d, J $=10.4 \mathrm{~Hz}, 1 \mathrm{H}, \mathbf{1 7}), 3.56(\mathrm{~d}, \mathrm{~J}=10.4 \mathrm{~Hz}, 1 \mathrm{H}, \mathbf{1 6}), 2.33(\mathrm{~s}, 3 \mathrm{H}, \mathbf{1 5}), 1.38(\mathrm{~d}, \mathrm{~J}=4.5 \mathrm{~Hz}, 1 \mathrm{H}, \mathbf{1 9}), 1.32(\mathrm{dd}, \mathrm{J}=4.6,1.2 \mathrm{~Hz}, 1 \mathrm{H}, \mathbf{1 8})$, $0.79(\mathrm{~s}, 3 \mathrm{H}, \mathbf{8})$.

${ }^{13} \mathrm{C}$ NMR $\left(\mathrm{CDCl}_{3}, 101 \mathrm{MHz} ; \boldsymbol{\delta} \mathbf{p p m}\right)$ :

$140.75(\mathrm{CH}=, \mathbf{6}), 138.66(\mathrm{C}, \mathbf{9}), 135.64(\mathrm{C}, \mathbf{1 2}), 128.87(2 \mathrm{xCH}, \mathbf{1 1}, \mathbf{1 3}), 128.75(2 \times \mathrm{XH}, \mathbf{1 0}, \mathbf{1 4}), 111.76(=\mathrm{CH}, \mathbf{5}), 66.98\left(\mathrm{CH}{ }_{2}, 2\right)$, $30.97(\mathrm{C}, 4), 26.64(\mathrm{C}, \mathbf{3}), 22.93\left(\mathrm{CH}_{2}, 7\right), 21.01\left(\mathrm{CH}_{3}, \mathbf{1 5}\right), 16.77\left(\mathrm{CH}_{3}, \mathbf{8}\right)$.

HRMS (EI) m/z : [M+H] $]^{+}$Calcd for $\mathrm{C}_{14} \mathrm{H}_{16} \mathrm{O}$ 201.1279; Found 201.1272

1-methyl-6-(o-tolyl)-3-oxabicyclo[4.1.0]hept-4-ene [2r]

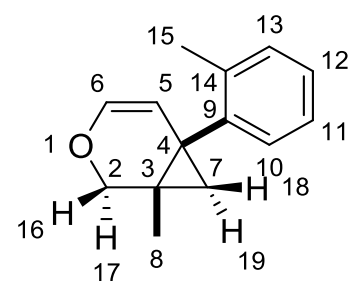

\author{
Formula : $\mathrm{C}_{14} \mathrm{H}_{16} \mathrm{O}$ \\ $\mathrm{M}: 200.28$ g.mol ${ }^{-1}$ \\ Aspect : Colorless oil \\ $\mathrm{R}_{\mathrm{f}}=0.5$ (Pentane ; Kagi-Mosher)
}

According to the experimental procedure (2) and starting from $84 \mathrm{mg}(0.42 \mathrm{mmol}, 1 \mathrm{eq}$.) of [1r], $5 \mathrm{mg}(8 \mu \mathrm{mol}, 2 \mathrm{~mol} \%)$ of IPrAuCl and $7 \mathrm{mg}(8 \mu \mathrm{mol}, 2 \mathrm{~mol} \%)$ of NaBAr ${ }^{\mathrm{F}}$, in Toluene $(0.5 \mathrm{M})$ and after $3 \mathrm{~h}$ of reaction, the 1-methyl-6-(o-tolyl)-3oxabicyclo[4.1.0]hept-4-ene [2r] was obtained as a colorless oil (82 $\mathrm{mg}, 98 \%)$. 
${ }^{1} \mathrm{H}$ NMR $\left(\mathrm{CDCl}_{3}, 400 \mathrm{MHz} ; \delta\right.$ ppm) :

$7.21(\mathrm{dq}, \mathrm{J}=5.8,3.8,2.6 \mathrm{~Hz}, 1 \mathrm{H}, \mathbf{1 1}), 7.19-7.09(\mathrm{~m}, 3 \mathrm{H}, \mathbf{1 0}, \mathbf{1 2}, \mathbf{1 3}), 6.16(\mathrm{~d}, \mathrm{~J}=5.8 \mathrm{~Hz}, 1 \mathrm{H}, \mathbf{6}), 5.07$ (d, J = 5.8 Hz, $1 \mathrm{H}, \mathbf{5}), 4.10$ $(\mathrm{d}, \mathrm{J}=10.5 \mathrm{~Hz}, 1 \mathrm{H}, \mathbf{1 7}), 3.75(\mathrm{~d}, \mathrm{~J}=10.5 \mathrm{~Hz}, 1 \mathrm{H}, \mathbf{1 6}), 2.36(\mathrm{~s}, 3 \mathrm{H}, \mathbf{1 5}), 1.46(\mathrm{~d}, \mathrm{~J}=4.3 \mathrm{~Hz}, 1 \mathrm{H}, \mathbf{1 9}), 1.25-1.23(\mathrm{~m}, 1 \mathrm{H}, 18), 0.86$ (s, 3H, 8).

${ }^{13} \mathrm{C}$ NMR $\left(\mathrm{CDCl}_{3}, 101 \mathrm{MHz} ; \delta\right.$ ppm) :

$140.11(\mathrm{CH}, \mathbf{6}), 139.79(\mathrm{C}, 9), 138.80(\mathrm{C}, \mathbf{1 4}), 130.27(\mathrm{CH}, \mathbf{1 3}), 129.90(\mathrm{CH}, \mathbf{1 2}), 126.75(\mathrm{CH}, \mathbf{1 1}), 125.57(\mathrm{CH}, 10), 111.85(\mathrm{CH}$, 5), $66.54\left(\mathrm{CH}_{2}, 2\right), 30.35(\mathrm{C}, 4), 27.62(\mathrm{C}, 3), 23.68\left(\mathrm{CH}_{2}, 7\right), 20.58\left(\mathrm{CH}_{3}, \mathbf{1 5}\right), 16.29\left(\mathrm{CH}_{3}, \mathbf{8}\right)$.

HRMS (EI) m/z : [M+H] $]^{+}$Calcd for $\mathrm{C}_{14} \mathrm{H}_{16} \mathrm{O}$ 201.1279; Found 201.1271

6-(4-bromophenyl)-1-methyl-3-oxabicyclo[4.1.0]hept-4-ene [2s]

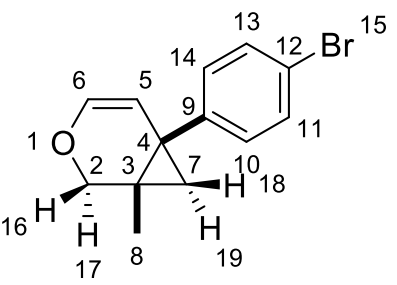

\author{
Formula : $\mathrm{C}_{13} \mathrm{H}_{13} \mathrm{BrO}$ \\ $\mathrm{M}: 265.15$ g.mol ${ }^{-1}$ \\ Aspect : Colorless oil \\ $\mathrm{R}_{\mathrm{f}}=0.47$ (Pentane ; Kagi-Mosher)
}

According to the experimental procedure (2) and starting from $139 \mathrm{mg}(0.52 \mathrm{mmol}, 1 \mathrm{eq}$.$) of [1s], 7 \mathrm{mg}(10.5 \mu \mathrm{mol}, 2 \mathrm{~mol} \%)$ of IPrAuCl and $9 \mathrm{mg}(10.5 \mu \mathrm{mol}, 2 \mathrm{~mol} \%)$ of $\mathbf{N a B A r} \mathbf{r}^{\mathrm{F}}$, in Toluene $(0.5 \mathrm{M})$ and after $3 \mathrm{~h}$ of reaction, the 6-(4-bromophenyl)-1methyl-3-oxabicyclo[4.1.0]hept-4-ene [2s] was obtained as a colorless oil (135 $\mathrm{mg}, 97$ \%).

${ }^{1} \mathrm{H}$ NMR $\left(\mathrm{CDCl}_{3}, 400 \mathrm{MHz} ; \delta\right.$ ppm) :

$7.42(\mathrm{~d}, J=8.5 \mathrm{~Hz}, 2 \mathrm{H}, 11,13), 7.13(\mathrm{~d}, J=8.5 \mathrm{~Hz}, 2 \mathrm{H}, 10,14), 6.21$ (d, J=5.8 Hz, 1H, 6), $5.14(\mathrm{~d}, J=5.8 \mathrm{~Hz}, 1 \mathrm{H}, 5), 4.06(\mathrm{~d}, J=10.5$ $\mathrm{Hz}, 1 \mathrm{H}, 17), 3.55(\mathrm{dd}, J=10.4,1.1 \mathrm{~Hz}, 1 \mathrm{H}, 16), 1.41(\mathrm{~d}, J=4.7 \mathrm{~Hz}, 1 \mathrm{H}, 19), 1.32(\mathrm{dd}, J=4.7,1.2 \mathrm{~Hz}, 1 \mathrm{H}, 18), 0.78(\mathrm{~s}, 3 \mathrm{H}, 8)$.

${ }^{13} \mathrm{C}$ NMR $\left(\mathrm{CDCl}_{3}, 101 \mathrm{MHz} ; \delta\right.$ ppm) :

$141.21(\mathrm{CH}=, 6), 140.82(\mathrm{C}, 9), 131.16(2 \times \mathrm{CH}, 11,13), 130.74(2 \times \mathrm{CH}, 10,14), 120.04(\mathrm{C}, 12), 110.80(=\mathrm{CH}, 5), 66.78(\mathrm{CH}, 2), 31.17$ (C, 4), $26.57(\mathrm{C}, 3), 23.10\left(\mathrm{CH}_{2}, 7\right), 16.76\left(\mathrm{CH}_{3}, 8\right)$.

HRMS (EI) m/z : [M+H $]^{+}$Calcd for $\mathrm{C}_{13} \mathrm{H}_{13} \mathrm{BrO} 265.0228$; Found 265.0218

1-bromo-3-(3-((2-methylallyl)oxy)prop-1-yn-1-yl)benzene [2t]

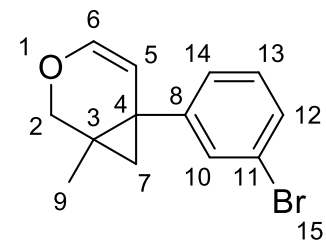

Formula : $\mathrm{C}_{13} \mathrm{H}_{13} \mathrm{BrO}$

$\mathrm{M}: 265.15 \mathrm{~g} \cdot \mathrm{mol}^{-1}$

Aspect : Light yellow oil

$\mathrm{R}_{\mathrm{f}}=0.72$ (petroleum ether/ $\mathrm{Et}_{2} \mathrm{O}, 98 / 2$; Kagi-Mosher)

$\mathrm{R}_{\mathrm{t}}=12.36 \mathrm{~min}$

According to the experimental procedure (2) and starting from $1.2 \mathrm{~g}(4.5 \mathrm{mmol}, 1$ eq., $\mathrm{M}=166.26)$ of [XXX], $3 \mathrm{mg}(5 \mu \mathrm{mol}, 0.1$ mol\%) of IPrAuCl and $4 \mathrm{mg}(5 \mu \mathrm{mol}, 0.1 \mathrm{~mol} \%)$ of $\mathbf{N a B A r}{ }^{\mathbf{F}}$, in Toluene $(0.5 \mathrm{M})$ the 6-pentyl-3-oxabicyclo[4.1.0]hept-4-ene [2t] was obtained after $2.5 \mathrm{~h}$ of reaction as a yellow oil $(1.14 \mathrm{~g}, 95 \%)$.

${ }^{1} \mathrm{H}$ NMR $\left(\mathrm{CDCl}_{3}, 400 \mathrm{MHz} ; \delta\right.$ ppm) :

$7.41(\mathrm{~s}, 1 \mathrm{H}, \mathbf{1 2}), 7.38-7.33(\mathrm{~m}, 1 \mathrm{H}, \mathbf{1 4}), 7.19-7.16(\mathrm{~m}, 2 \mathrm{H}, \mathbf{1 0}, \mathbf{1 3}), 6.22(\mathrm{~d}, J=5.8 \mathrm{~Hz}, 1 \mathrm{H}, \mathbf{6}), 5.16(\mathrm{~d}, J=5.8 \mathrm{~Hz}, 1 \mathrm{H}, \mathbf{5}), 4.07$ $\left(\mathrm{d}, J=10.5 \mathrm{~Hz}, 1 \mathrm{H}, \mathbf{2}^{\prime \prime}\right), 3.55$ (d, $\left.J=10.5 \mathrm{~Hz}, 1 \mathrm{H}, \mathbf{2}^{\prime}\right), 1.42$ (d, $\left.J=4.7 \mathrm{~Hz}, 1 \mathrm{H}, \mathbf{7}^{\prime \prime}\right), 1.36-1.33$ (m, 2H, 7'), 0.80 (s, 3H, 9).

${ }^{13} \mathrm{C}$ NMR $\left(\mathrm{CDCl}_{3}, 101 \mathrm{MHz} ; \delta\right.$ ppm) :

144.24 (CH, 6), 141.29 (C, 8), $132.14(\mathrm{CH}, 12), 129.63(\mathrm{CH}, \mathbf{1 0}), 129.36(\mathrm{CH}, 13), 127.64(\mathrm{CH}, 14), 122.29(\mathrm{C}, 11), 110.68(\mathrm{CH}$, 5), $66.77\left(\mathrm{CH}_{2}, 2\right), 31.39(\mathrm{C}, 4), 26.84(\mathrm{C}, 3), 23.14\left(\mathrm{CH}_{2}, 7\right), 16.78\left(\mathrm{CH}_{3}, 9\right)$.

HRMS (EI) m/z: $[\mathrm{M}+\mathrm{H}]^{+}$Calcd for $\mathrm{C}_{13} \mathrm{H}_{13} \mathrm{BrO}$ 265.0228; Found 265.0220 


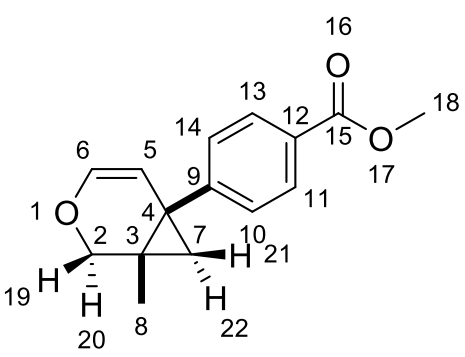

\author{
Formula : $\mathrm{C}_{15} \mathrm{H}_{16} \mathrm{O}_{3}$ \\ $\mathrm{M}: 244.29{\mathrm{~g} . \mathrm{mol}^{-1}}^{-1}$ \\ Aspect : white crystal \\ $\mathrm{R}_{\mathrm{f}}=0.47$ (Pentane/Et ${ }_{2} \mathrm{O}, 98 / 2$; Kagi-Mosher)
}

According to the experimental procedure (2) and starting from $374 \mathrm{mg}$ (1.5 mmol, 1 eq.) of [1u], $19 \mathrm{mg}(31 \mu \mathrm{mol}, 2 \mathrm{~mol} \%)$ of IPrAuCl and $27 \mathrm{mg}(31 \mu \mathrm{mol}, 2 \mathrm{~mol} \%)$ of $\mathbf{N a B A r}{ }^{\mathrm{F}}$, in Toluene $(0.5 \mathrm{M})$ and after $2 \mathrm{~h}$ of reaction, the methyl 4-(1-methyl-3oxabicyclo[4.1.0] hept-4-en-6-yl)benzoate [2u] was obtained after precipitation in DCM/petroleum ether (1/10) as a white crystal $(226 \mathrm{mg}, 60 \%)$.

${ }^{1} \mathrm{H}$ NMR $\left(\mathrm{CDCl}_{3}, 400 \mathrm{MHz} ; \delta\right.$ ppm) : $8.05-7.94(\mathrm{~m}, 2 \mathrm{H}, \mathbf{1 1}, 13), 7.36-7.27(\mathrm{~m}, 2 \mathrm{H}, 1 \mathbf{1 0}, 14), 6.25(\mathrm{~d}, \mathrm{~J}=5.8 \mathrm{~Hz}, 1 \mathrm{H}, 6), 5.19(\mathrm{~d}, \mathrm{~J}=5.8 \mathrm{~Hz}, 1 \mathrm{H}, 5), 4.08(\mathrm{~d}, \mathrm{~J}=10.5 \mathrm{~Hz}$, $1 \mathrm{H}, 20), 3.91(\mathrm{~s}, 3 \mathrm{H}, 18), 3.57(\mathrm{~d}, \mathrm{~J}=10.6 \mathrm{~Hz}, 1 \mathrm{H}, 19), 1.46(\mathrm{~d}, \mathrm{~J}=4.8 \mathrm{~Hz}, 1 \mathrm{H}, 22), 1.43(\mathrm{dd}, \mathrm{J}=4.7,1.1 \mathrm{~Hz}, 1 \mathrm{H}, 21), 0.78(\mathrm{~s}, 3 \mathrm{H}, 8)$.

${ }^{13} \mathrm{C}$ NMR $\left(\mathrm{CDCl}_{3}, 101 \mathrm{MHz} ; \delta\right.$ ppm) :

$167.05(\mathrm{C}=\mathrm{O}, 15), 147.17(\mathrm{C}, 9), 141.40(\mathrm{CH}=, 6), 129.39(2 \times \mathrm{CH}, 11,13), 128.91(2 \times C H, 10,14), 128.06(\mathrm{C}, 12), 110.48(=\mathrm{CH}, 5)$, $66.75(\mathrm{CH} 2,2), 52.03(\mathrm{CH} 3,18), 31.71(\mathrm{C}, 4), 27.12(\mathrm{C}, 3), 23.21(\mathrm{CH} 2,7), 16.62(\mathrm{CH} 3,8)$.

HRMS (EI) m/z : [M+H] $]^{+}$Calcd for $\mathrm{C}_{15} \mathrm{H}_{16} \mathrm{O}_{3}$ 245.1178; Found 245.1169

4-(1-methyl-3-oxabicyclo[4.1.0]hept-4-en-6-yl)benzonitrile [2v]

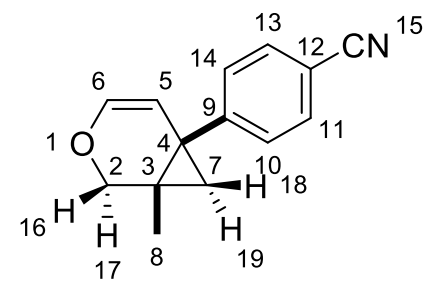

\author{
Formula : $\mathrm{C}_{14} \mathrm{H}_{13} \mathrm{NO}$ \\ $\mathrm{M}: 211.26 \mathrm{~g} \cdot \mathrm{mol}^{-1}$ \\ Aspect : Colorless oil \\ $\mathrm{R}_{\mathrm{f}}=0.76$ (Pentane/Et ${ }_{2} \mathrm{O}, 80 / 20 ;$ Kagi-Mosher)
}

According to the experimental procedure (2) and starting from $209 \mathrm{mg}(0.99 \mathrm{mmol}, 1 \mathrm{eq}$.$) of [1v], 12 \mathrm{mg}(20 \mu \mathrm{mol}, 2 \mathrm{~mol} \%)$ of IPrAuCl and $18 \mathrm{mg}(20 \mu \mathrm{mol}, 2 \mathrm{~mol} \%)$ of $\mathbf{N a B A r} \mathbf{F}^{\mathbf{F}}$, in Toluene $(0.5 \mathrm{M})$ and after $28 \mathrm{~h}$ of reaction, the 4-(1-methyl-3oxabicyclo[4.1.0]hept-4-en-6-yl)benzonitrile [2v] was obtained as an Colorless oil (85 mg, $41 \%)$.

${ }^{1} \mathrm{H}$ NMR $\left(\mathrm{CDCl}_{3}, 400 \mathrm{MHz} ; \delta\right.$ ppm) :

$7.64-7.57(\mathrm{~m}, 2 \mathrm{H}, 11,13), 7.43-7.29(\mathrm{~m}, 2 \mathrm{H}, 10,14), 6.26(\mathrm{~d}, \mathrm{~J}=5.9 \mathrm{~Hz}, 1 \mathrm{H}, 6), 5.14(\mathrm{~d}, \mathrm{~J}=5.8 \mathrm{~Hz}, 1 \mathrm{H}, 5), 4.09(\mathrm{~d}, \mathrm{~J}=10.6 \mathrm{~Hz}$, $1 \mathrm{H}, 17), 3.56(\mathrm{~d}, \mathrm{~J}=10.6 \mathrm{~Hz}, 1 \mathrm{H}, 16), 1.49(\mathrm{~d}, \mathrm{~J}=4.9 \mathrm{~Hz}, 1 \mathrm{H}, 19), 1.42(\mathrm{dd}, \mathrm{J}=4.8,1.2 \mathrm{~Hz}, 1 \mathrm{H}, 18), 0.78(\mathrm{~s}, 3 \mathrm{H}, 8)$.

${ }^{13} \mathrm{C}$ NMR $\left(\mathrm{CDCl}_{3}, 101 \mathrm{MHz} ; \delta\right.$ ppm) :

147.43 (C, 9), $141.84(\mathrm{CH}, 6), 131.93$ (2xCH, 11, 13), 129.65 (2xCH, 10, 14), 118.96 (CN, 15), 109.99 (C, 12$), 109.73$ (CH, 5), 66.61 $\left(\mathrm{CH}_{2}, 2\right), 31.97(\mathrm{C}, 4), 27.22(\mathrm{C}, 3), 23.32\left(\mathrm{CH}_{2}, 7\right), 16.67\left(\mathrm{CH}_{3}, 8\right)$.

HRMS (EI) m/z: $[\mathrm{M}+\mathrm{H}]^{+}$Calcd for $\mathrm{C}_{14} \mathrm{H}_{13} \mathrm{NO}$ 212.1075; Found 212.1070

\title{
III. Derivatization of $[2 t]$
}

1-(3-(1-methyl-3-oxabicyclo[4.1.0]hept-4-en-6-yl)phenyl)cyclobutan-1-ol [4]

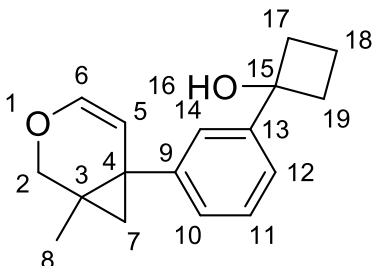

Formula : $\mathrm{C}_{17} \mathrm{H}_{20} \mathrm{O}_{2}$ $\mathrm{M}: 256.35 \mathrm{~g} . \mathrm{mol}^{-1}$

Aspect : white solid

$\mathrm{R}_{\mathrm{f}}=0.3$ (petroleum ether/ $\mathrm{Et}_{2} \mathrm{O}, 50 / 50$; Kagi-Mosher) 
Mg powder (1.5 eq, $0.57 \mathrm{mmol}, 150 \mathrm{mg}), \mathbf{I}_{2}(5 \mathrm{mg})$ and $1.7 \mathrm{~mL}$ of dry THF $(0.75 \mathrm{ml})$ were stirred until the yellow color of iodine disappeared. A small quantity of the halide [2t] $(1 \mathrm{eq}, 0.57 \mathrm{mmol})$ in THF $(0.75 \mathrm{ml})$ was added and the flask was carefully heated to initiate the reaction. When the reaction mixture became less cloudy, halide was added dropwise. At the end of the addition, the mixture should be a clear white solution (reaction monitored by $\mathrm{TLC}$, work up with $\mathrm{NH}_{4} \mathrm{Cl} / \mathrm{Et}_{2} \mathrm{O}$ ). After the addition, reaction mixture is stirred for 30 minutes and cyclobutanone is added (1.5 eq, $0.85 \mathrm{mmol}, 65 \mu \mathrm{L})$. Reaction mixture is stirred for another 1 hour and quenched with saturated solution of $\mathrm{NH}_{4} \mathrm{Cl}$ and the product was extracted thrice with $\mathrm{Et}_{2} \mathrm{O}$. The combined organic layers were dried over $\mathrm{MgSO}_{4}$, the solvent was removed under reduced pressure and the titled compound 1-(3-(1-methyl-3oxabicyclo[4.1.0]hept-4-en-6-yl)phenyl)cyclobutan-1-ol [4]was obtain after FCC as a white solid (100 mg, 69 \%).

${ }^{1} \mathrm{H}$ NMR $\left(\mathrm{CDCl}_{3}, 400 \mathrm{MHz} ; \delta\right.$ ppm) :

$7.42-7.27(\mathrm{~m}, 3 \mathrm{H}, \mathbf{1 1}, \mathbf{1 2}, \mathbf{1 4}), 7.18(\mathrm{dt}, J=7.3,1.6 \mathrm{~Hz}, 1 \mathrm{H}, \mathbf{1 0}), 6.22(\mathrm{~d}, J=5.8 \mathrm{~Hz}, 1 \mathrm{H}, \mathbf{6}), 5.21(\mathrm{~d}, J=5.8 \mathrm{~Hz}, 1 \mathrm{H}, \mathbf{5}), 4.08(\mathrm{~d}$, $\left.J=10.5 \mathrm{~Hz}, 1 \mathrm{H}, 2^{\prime}\right), 3.58$ (d, $\left.J=10.5 \mathrm{~Hz}, 1 \mathrm{H}, \mathbf{2}^{\prime \prime}\right), 2.63-2.52$ (m, 2H, 17', 19'), $2.45-2.31$ (m, 2H, 17', 19'), 2.04 (dddd, $J=$ $\left.11.2,9.4,5.3,4.0 \mathrm{~Hz}, 1 \mathrm{H}, \mathbf{1 8}^{\prime \prime}\right), 1.97$ (s, 1H, 16), 1.70 (dtt, $\left.J=11.2,8.8,7.4 \mathrm{~Hz}, 1 \mathrm{H}, \mathbf{1 8}^{\prime}\right), 1.42$ (d, $\left.J=4.7 \mathrm{~Hz}, 1 \mathrm{H}, 7^{\prime \prime}\right), 1.38$ (dd, $J$ $\left.=4.6,1.1 \mathrm{~Hz}, 1 \mathrm{H}, 7^{\prime}\right), 0.80(\mathrm{~s}, 3 \mathrm{H}, \mathbf{8})$.

${ }^{13} \mathrm{C}$ NMR $\left(\mathrm{CDCl}_{3}, 101 \mathrm{MHz} ; \delta\right.$ ppm) :

$146.11(\mathrm{CH}, \mathbf{6}), 141.96(\mathrm{C}, \mathbf{1 3}), 140.93(\mathrm{C}, \mathbf{9}), 128.13(\mathrm{CH}, \mathbf{1 0}), 127.97(\mathrm{CH}, \mathbf{1 1}), 125.53(\mathrm{CH}, 12), 122.77(\mathrm{CH}, 14), 111.45(\mathrm{CH}$, 5), $77.08(\mathrm{C}, \mathbf{1 5}), 66.92\left(\mathrm{CH}_{2}, 2\right), 36.93\left(\mathrm{CH}_{2}, \mathbf{1 7}\right), 36.91\left(\mathrm{CH}_{2}, \mathbf{1 9}\right), 31.11(\mathrm{C}, 4), 27.16(\mathrm{C}, \mathbf{3}), 23.10\left(\mathrm{CH}_{2}, 7\right), 16.86\left(\mathrm{CH}_{3}, \mathbf{8}\right)$, $13.06\left(\mathrm{CH}_{2}, \mathbf{1 8}\right)$.

HRMS (EI) $\mathbf{m} / \mathbf{z}:[\mathrm{M}+\mathrm{H}]^{+}$Calcd for $\mathrm{C}_{17} \mathrm{H}_{20} \mathrm{O}_{2}$ 257.1542; Found 257.1532

\section{Derivatization of $[2 \mathrm{~g}]$}

1-methyl-6-phenyl-3-oxabicyclo[4.1.0]heptane [5]

To a solution of 4-oxabicyclo[4.1.0]hexene [2g] (1 eq, $86 \mathrm{mmol}, 16.02 \mathrm{~g})$ and Triethylsilane (3 eq, $258 \mathrm{mmol}, 20.13 \mathrm{~mL})$ in DCM $(0.2 \mathrm{M})$ at $0^{\circ} \mathrm{C}$ is added Trifluoroacetic acid $(1.05 \mathrm{eq}, 90 \mathrm{mmol}, 14.2 \mathrm{~mL})$. Reaction mixture is stirred for $30 \mathrm{minutes}$ until completion. Reaction mixture is diluted in ca $20 \mathrm{~mL}$ of water and extracted thrice with DCM ( $3 \times 30 \mathrm{ml})$ dried over $\mathrm{MgSO}_{4}$, concentrated in vacuuo and distilled $\left(142^{\circ} \mathrm{C}\right.$ at $\left.0.5 \mathrm{mBar}\right)$ to afford 1-methyl-6-phenyl-3-oxabicyclo[4.1.0]heptane [5] as a colorless oil $(14.8 \mathrm{~g}, 91 \%)$

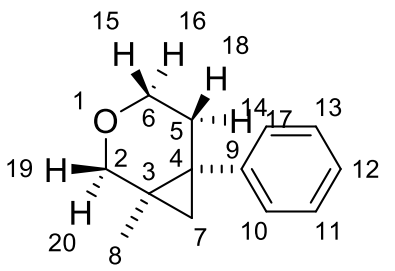

Formula : $\mathrm{C}_{13} \mathrm{H}_{16} \mathrm{O}$

$\mathrm{M}: 188.27 \mathrm{~g} \cdot \mathrm{mol}^{-1}$

Aspect : colorless oil

$\mathrm{R}_{\mathrm{f}}=0.58$ (petroleum ether/Et ${ }_{2} \mathrm{O}, 90 / 10 ;$ Kagi-Mosher)

${ }^{1} \mathrm{H}$ NMR $\left(\mathrm{CDCl}_{3}, 400 \mathrm{MHz} ; \delta\right.$ ppm) :

$7.34-7.25(\mathrm{~m}, 2 \mathrm{H}, \mathbf{1 0}, \mathbf{1 4}), 7.26-7.14(\mathrm{~m}, 3 \mathrm{H}, \mathbf{1 1}, \mathbf{1 2}, \mathbf{1 3}), 3.93(\mathrm{~d}, J=11.2 \mathrm{~Hz}, 1 \mathrm{H}, \mathbf{1 9}), 3.77$ (ddd, $J=11.4,6.4,2.6 \mathrm{~Hz}, 1 \mathrm{H}$, 15), $3.58(\mathrm{~d}, J=11.2 \mathrm{~Hz}, 1 \mathrm{H}, \mathbf{2 0}), 3.43(\mathrm{td}, J=11.2,5.0 \mathrm{~Hz}, 1 \mathrm{H}, \mathbf{1 6}), 2.13(\mathrm{ddd}, J=14.4,10.9,6.4 \mathrm{~Hz}, 1 \mathrm{H}, 18), 1.89(\mathrm{ddd}, J=$ $14.3,5.1,2.7 \mathrm{~Hz}, 1 \mathrm{H}, 17), 0.97(\mathrm{~s}, 1 \mathrm{H}, 7), 0.72(\mathrm{~s}, 3 \mathrm{H}, 8)$.

${ }^{13} \mathrm{C}$ NMR $\left(\mathrm{CDCl}_{3}, 101 \mathrm{MHz} ; \delta\right.$ ppm) :

$145.51(\mathrm{C}, \mathbf{9}), 129.05(2 \mathrm{xCH}, \mathbf{1 1}, \mathbf{1 3}), 128.24(2 \mathrm{xCH}, \mathbf{1 0}, \mathbf{1 4}), 125.94(\mathrm{CH}, \mathbf{1 2}), 71.19\left(\mathrm{CH}_{2}, \mathbf{2}\right), 65.81\left(\mathrm{CH}_{2}, \mathbf{6}\right), 33.46\left(\mathrm{CH}_{2}, \mathbf{5}\right)$, $28.75(\mathrm{C}, 4), 21.82\left(\mathrm{CH}_{2}, 7\right), 21.14(\mathrm{C}, \mathbf{3}), 18.95\left(\mathrm{CH}_{3}, \mathbf{8}\right)$.

HRMS (EI) m/z : [M+H] $]^{+}$Calcd for $\mathrm{C}_{13} \mathrm{H}_{16} \mathrm{O}$ 189.1279; Found 189.1272

(2-formyl-1-methyl-2-phenylcyclopropyl)methyl formate [6]

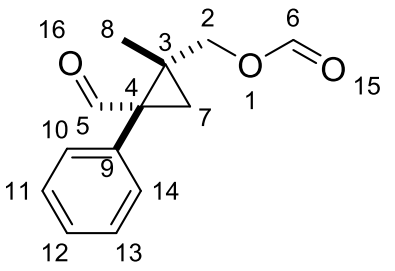

Formule Chimique : $\mathrm{C}_{13} \mathrm{H}_{14} \mathrm{O}_{3}$

Masse moléculaire : $218.25 \mathrm{~g} \cdot \mathrm{mol}^{-1}$

Aspect : Colorless oil

$\mathrm{R}_{\mathrm{f}}=0.36$ (éther de pétrole $/ \mathrm{Et}_{2} \mathrm{O}, 90 / 10 ; \mathrm{Kagi}$ ) 
To a solution of 4-oxabicyclo[4.1.0]hexene (1 eq, $1.61 \mathrm{mmol}, 300 \mathrm{mg})$ in $\mathbf{D C M}(0.025 \mathrm{M})$ at $-78^{\circ} \mathrm{C}$ is bubbled $\mathbf{O}_{3}$ at $0.8 \mathrm{~A}$. Reaction mixture is stirred for 7 minutes until appearance of the blue color of Ozone (indicating the completion of the reaction).

Dimethyl sulfide ( 2 eq, $3.22 \mathrm{mmol}, 236 \mu \mathrm{L}$ ) is added and the solvent is removed. Reaction mixture is diluted in ca $20 \mathrm{~mL}$ of water and extracted thrice with EtOAc $(3 \times 30 \mathrm{ml})$ then washed with brine, dried over $\mathrm{MgSO}_{4}$ and concentrated in vacuuo to afford titled compound (2-formyl-1-methyl-2-phenylcyclopropyl)methyl formate [6] as an Colorless oil (250 mg, $71 \%$ )

${ }^{1} \mathrm{H}$ NMR $\left(\mathrm{CDCl}_{3}, 400 \mathrm{MHz} ; \delta\right.$ ppm) :

$9.76(\mathrm{~s}, 1 \mathrm{H}, \mathbf{5}), 8.09$ (s, 1H, 6), $7.47-7.38$ (m, 2H, 10, 14), $7.38-7.29$ (m, 3H, 11, 12, 13), 4.54 (dd, J = 11.7, $1.0 \mathrm{~Hz}, 1 \mathrm{H}, 2$ ") , $4.16\left(\mathrm{~d}, J=11.7 \mathrm{~Hz}, 1 \mathrm{H}, 2^{\prime}\right), 2.02\left(\mathrm{~d}, J=5.0 \mathrm{~Hz}, 1 \mathrm{H}, 7^{\prime}\right), 1.47$ (d, $\left.J=5.0 \mathrm{~Hz}, 1 \mathrm{H}, 7^{\prime \prime}\right), 0.91$ (s, 3H, 8).

${ }^{13} \mathrm{C}$ NMR $\left(\mathrm{CDCl}_{3}, 101 \mathrm{MHz} ; \delta \mathrm{ppm}\right)$ :

$200.24(\mathrm{CHO}, \mathbf{5}), 160.67(\mathrm{HCOO}, \mathbf{6}), 135.75(\mathrm{C}, 9), 130.52(2 \mathrm{xCH}, \mathbf{1 1}, \mathbf{1 3}), 128.87(2 \mathrm{xCH}, \mathbf{1 0}, 14), 127.78\left(\mathrm{CH}_{12}, 12\right), 65.52\left(\mathrm{CH}_{2}\right.$, 2), $46.55(\mathrm{C}, 4), 33.95(\mathrm{C}, 3), 23.84\left(\mathrm{CH}_{2}, 7\right), 19.65\left(\mathrm{CH}_{3}, 8\right)$.

HRMS (EI) $\mathbf{m} / \mathbf{z}:[\mathrm{M}+\mathrm{H}]^{+}$Calcd for $\mathrm{C}_{13} \mathrm{H}_{14} \mathrm{O}_{3}$ 218.0943; Found 218.0936

(1S,2R,5S)-2-methoxy-5-methyl-1-phenyl-3-oxabicyclo[3.1.0]hexane / (1R,2S,5R)-2-methoxy-5-methyl-1-phenyl-3oxabicyclo[3.1.0]hexane [7]

To a solution of (2-formyl-1-methyl-2-phenylcyclopropyl)methyl formate [6] (1 eq, $2 \mathrm{mmol}, 444 \mathrm{mg})$ in $\mathrm{MeOH}(0.25 \mathrm{M})$ is added APTS $(0.1 \mathrm{eq}, 0.2 \mathrm{mmol}, 35 \mathrm{mg})$. Reaction mixture is stirred at $40^{\circ} \mathrm{C}$ for 4 hours until completion monitored by TLC. Reaction mixture is then concentrated in vacuuo and purified on flash chromatography packed with $1 \% \mathrm{Et}_{3} \mathrm{~N}$ to isolate [7] (258 $\mathrm{mg}, 62 \%)$ as a white solid.

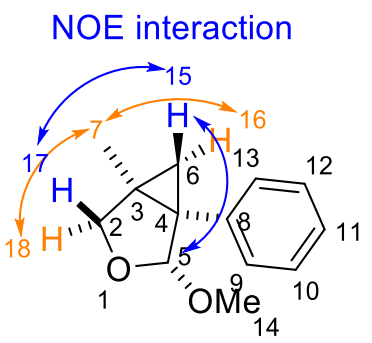

\author{
Formula : $\mathrm{C}_{13} \mathrm{H}_{16} \mathrm{O}_{2}$ \\ Molecular mass : $204.27 \mathrm{~g} \cdot \mathrm{mol}^{-1}$ \\ Aspect : white solid \\ $\mathrm{R}_{\mathrm{f}}=0.76$ (petroleum ether $/ \mathrm{Et}_{2} \mathrm{O}, 80 / 20 ;$ Kagi)
}

${ }^{1} \mathrm{H}$ NMR $\left(\mathrm{CDCl}_{3}, 400 \mathrm{MHz} ; \delta\right.$ ppm) :

$7.39-7.33(\mathrm{~m}, 2 \mathrm{H}, \mathbf{1 0}, \mathbf{1 2}), 7.33-7.30(\mathrm{~m}, 1 \mathrm{H}, \mathbf{1 1}), 7.30-7.20(\mathrm{~m}, 2 \mathrm{H}, \mathbf{9}, \mathbf{1 3}), 4.79(\mathrm{~s}, 1 \mathrm{H}, \mathbf{5}), 3.91(\mathrm{~d}, \mathrm{~J}=7.9 \mathrm{~Hz}, 1 \mathrm{H}, \mathbf{1 8}), 3.81$ $(\mathrm{d}, J=7.8 \mathrm{~Hz}, 1 \mathrm{H}, 17), 3.27(\mathrm{~s}, 3 \mathrm{H}, 19), 1.08(\mathrm{~s}, 3 \mathrm{H}, 7), 0.99(\mathrm{~d}, J=4.3 \mathrm{~Hz}, 1 \mathrm{H}, 15), 0.76(\mathrm{dd}, J=4.4,0.8 \mathrm{~Hz}, 1 \mathrm{H}, 16)$.

${ }^{13} \mathrm{C}$ NMR $\left(\mathrm{CDCl}_{3}, 101 \mathrm{MHz} ; \delta\right.$ ppm) :

$136.20(\mathrm{C}, \mathbf{8}), 132.15(2 \mathrm{xCH}, \mathbf{1 0}, \mathbf{1 2}), 127.64(2 \mathrm{xCH}, \mathbf{9}, \mathbf{1 3}), 126.86(\mathrm{CH}, \mathbf{1 1}), 107.57(\mathrm{CH}, \mathbf{5}), 72.34\left(\mathrm{CH}_{2}, 2\right), 54.96(\mathrm{OMe}, \mathbf{1 9})$, $39.98(\mathrm{C}, 4), 27.26(\mathrm{C}, \mathbf{3}), 19.45\left(\mathrm{CH}_{2}, \mathbf{6}\right), 14.35\left(\mathrm{CH}_{3}, 7\right)$.

HRMS (EI) m/z: $[\mathrm{M}+\mathrm{H}]^{+}$Calcd for $\mathrm{C}_{13} \mathrm{H}_{16} \mathrm{O}_{2}$ 205.1229; Found 205.1224

(1R,4R,6R)-4-methoxy-1-methyl-6-phenyl-3-oxabicyclo[4.1.0]heptane and (1S,4S,6S)-4-methoxy-1-methyl-6-phenyl-3oxabicyclo[4.1.0]heptane [8a]

To a solution of 4-oxabicyclo[4.1.0]hexene (1 eq, $0.96 \mathrm{mmol}, 179 \mathrm{mg})$ in $\mathrm{MeOH}(0.5 \mathrm{M})$ at $\mathrm{rt}$ is added TFA (2 eq, $1.93 \mathrm{mmol}, 147$ $\mu \mathrm{L})$. Reaction mixture is stirred for 4 days until complete disappearance of the enol ether. A saturated solution of $\mathrm{NaHCO}_{3}$ is added and aqueaous phase is extracted thrice with DCM. Combined organic phases are dried over $\mathrm{MgSO}_{4}$ and concentrated in vacuuo. Crude is purified on a silica column to afford 2 diastereoisomers, [8a] (96 mg, 45\%) as a colorless oil and [8b] (58 mg, $28 \%$ ) as a colorless oil.

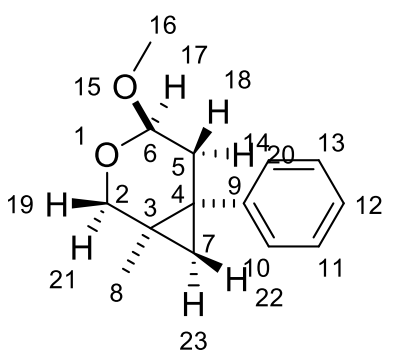

Formula : $\mathrm{C}_{14} \mathrm{H}_{18} \mathrm{O}_{2}$

Molecular Mass : 218.30 g. $\mathrm{mol}^{-1}$

Aspect : Colorless oil

$\mathrm{R}_{\mathrm{f}}=0.63$ (petroleum ether/ $\mathrm{Et}_{2} \mathrm{O}, 90 / 10$; Kagi) 
${ }^{1} \mathrm{H}$ NMR $\left(\mathrm{CDCl}_{3}, 400 \mathrm{MHz} ; \delta\right.$ ppm) :

$7.28(\mathrm{~d}, J=4.5 \mathrm{~Hz}, 4 \mathrm{H}, 10,11,13,14), 7.18$ (ddd, $J=8.7,5.0,3.8 \mathrm{~Hz}, 1 \mathrm{H}, 12$ ), 4.59 (dd, $J=5.7$ [ax-eq], 2.1 [eq-eq] Hz, $1 \mathrm{H}, 17$ ), 3.80 (d, $J=11.1 \mathrm{~Hz}, 1 \mathrm{H}, 19), 3.64$ (d, J = $11.1 \mathrm{~Hz}, 1 \mathrm{H}, 21), 3.40$ (s, 3H, 16), 2.23 (dd, J = 15.0, 5.7 [ax-eq] Hz, 1H, 20), 1.98 (ddd, J = 15.0, 2.1 [eq-eq], $1.0 \mathrm{~Hz}, 1 \mathrm{H}, 18), 0.97(\mathrm{~d}, J=4.6 \mathrm{~Hz}, 1 \mathrm{H}, 23), 0.94(\mathrm{dd}, J=4.6,1.0 \mathrm{~Hz}, 1 \mathrm{H}, 22), 0.69(\mathrm{~s}, 3 \mathrm{H}, 8)$.

${ }^{13} \mathrm{C}$ NMR $\left(\mathrm{CDCl}_{3}, 101 \mathrm{MHz} ; \delta \mathrm{ppm}\right)$ :

$144.75(\mathrm{C}, 9), 129.39(2 \times C H, 11,13), 128.09(2 \times C H, 10,14), 125.86(\mathrm{CH}, 12), 96.51(\mathrm{CH}-\mathrm{OMe}, 6), 61.41\left(\mathrm{CH}_{2}, 2\right), 54.91(\mathrm{OCH}, 16)$, $37.02\left(\mathrm{CH}_{2}, 5\right), 26.04(\mathrm{C}, 4), 20.36\left(\mathrm{CH}_{2}, 7\right), 19.57(\mathrm{C}, 3), 18.03\left(\mathrm{CH}_{3}, 8\right)$.

HRMS (EI) m/z : [M+H] $]^{+}$Calcd for $\mathrm{C}_{14} \mathrm{H}_{18} \mathrm{O}_{2}$ 219.1385; Found 219.1380

(1R,4S,6R)-4-methoxy-1-methyl-6-phenyl-3-oxabicyclo[4.1.0]heptane and (1S,4R,6S)-4-methoxy-1-methyl-6-phenyl-3oxabicyclo[4.1.0]heptane [8b]

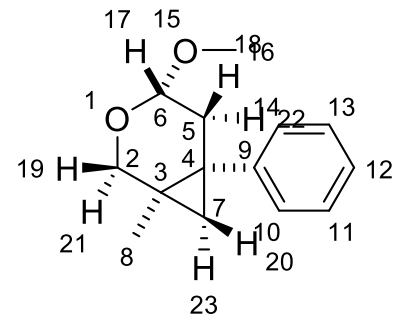

Formula : $\mathrm{C}_{14} \mathrm{H}_{18} \mathrm{O}_{2}$

Molecular Mass : 218.30 g. $\mathrm{mol}^{-1}$

Aspect : Colorless oil

$\mathrm{R}_{\mathrm{f}}=0.48$ (petroleum ether $/ \mathrm{Et}_{2} \mathrm{O}, 90 / 10$; Kagi)

${ }^{1} \mathrm{H}$ NMR $\left(\mathrm{CDCl}_{3}, 400 \mathrm{MHz} ; \delta\right.$ ppm) :

7.29 (ddd, $J=7.8,6.6,1.8 \mathrm{~Hz}, 2 \mathrm{H}, 11,13), 7.23-7.14(\mathrm{~m}, 3 \mathrm{H}, 10,12,14), 4.46$ (dd, $J=8.8$ [ax-ax], 4.4 [ax-eq] Hz, 1H, 17), 4.03 (d, $J=11.5 \mathrm{~Hz}, 1 \mathrm{H}, 21), 3.70(\mathrm{~d}, J=11.5 \mathrm{~Hz}, 1 \mathrm{H}, 19), 3.46(\mathrm{~s}, 3 \mathrm{H}, 16), 2.13$ (ddd, J=14.5, 4.4 [ax-eq], $0.9 \mathrm{~Hz}, 1 \mathrm{H}, 18), 1.95$ (dd, $J=$ 14.5, $8.8[a x-a x] ~ H z, 1 H, 22), 1.04(d, J=4.5 \mathrm{~Hz}, 1 \mathrm{H}, 23), 1.00(\mathrm{~d}, J=4.5 \mathrm{~Hz}, 1 \mathrm{H}, 20), 0.71(\mathrm{~s}, 3 \mathrm{H}, 8)$.

${ }^{13} \mathrm{C} \mathrm{NMR}\left(\mathrm{CDCl}_{3}, 101 \mathrm{MHz} ; \delta\right.$ ppm) :

144.80 (C, 9), $129.03(2 x \mathrm{CH}, 11,13), 128.27(2 \times \mathrm{CH}, 10,14), 126.04(\mathrm{CH}, 12), 101.71(\mathrm{CH}-\mathrm{OMe}, 6), 69.30\left(\mathrm{CH}_{2}, 2\right), 55.96\left(\mathrm{OCH}_{3}, 16\right)$, $38.76\left(\mathrm{CH}_{2}, 5\right), 28.05(\mathrm{C}, 4), 21.72\left(\mathrm{CH}_{2}, 7\right), 21.28(\mathrm{C}, 3), 18.17\left(\mathrm{CH}_{3}, 8\right)$.

HRMS (EI) $\mathbf{m} / \mathbf{z}:[\mathrm{M}+\mathrm{H}]^{+}$Calcd for $\mathrm{C}_{14} \mathrm{H}_{18} \mathrm{O}_{2}$ 219.1385; Found 219.1380 


\section{NMR Spectra}

1. NMR spectra of 1,6-enynes

[1e]
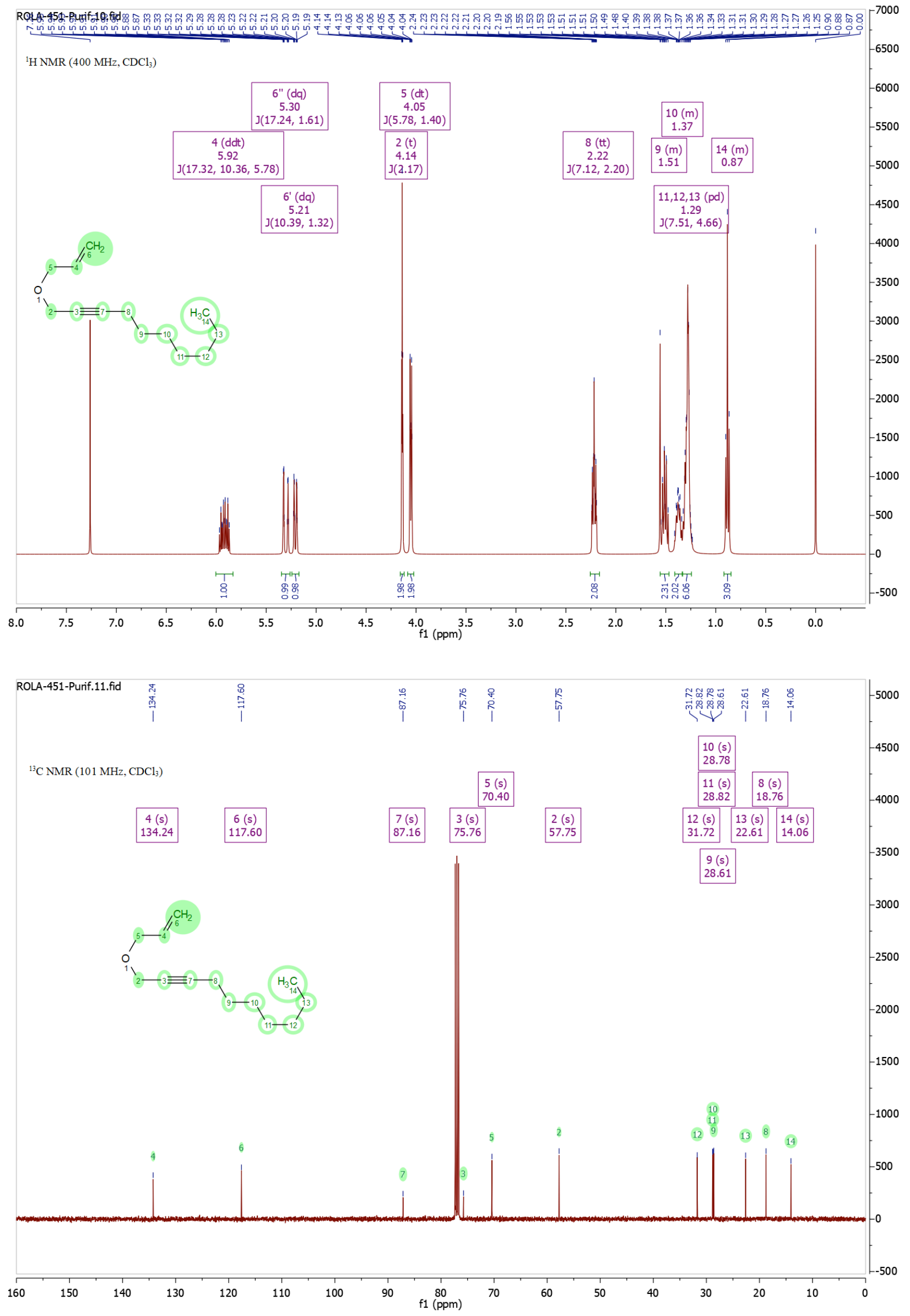

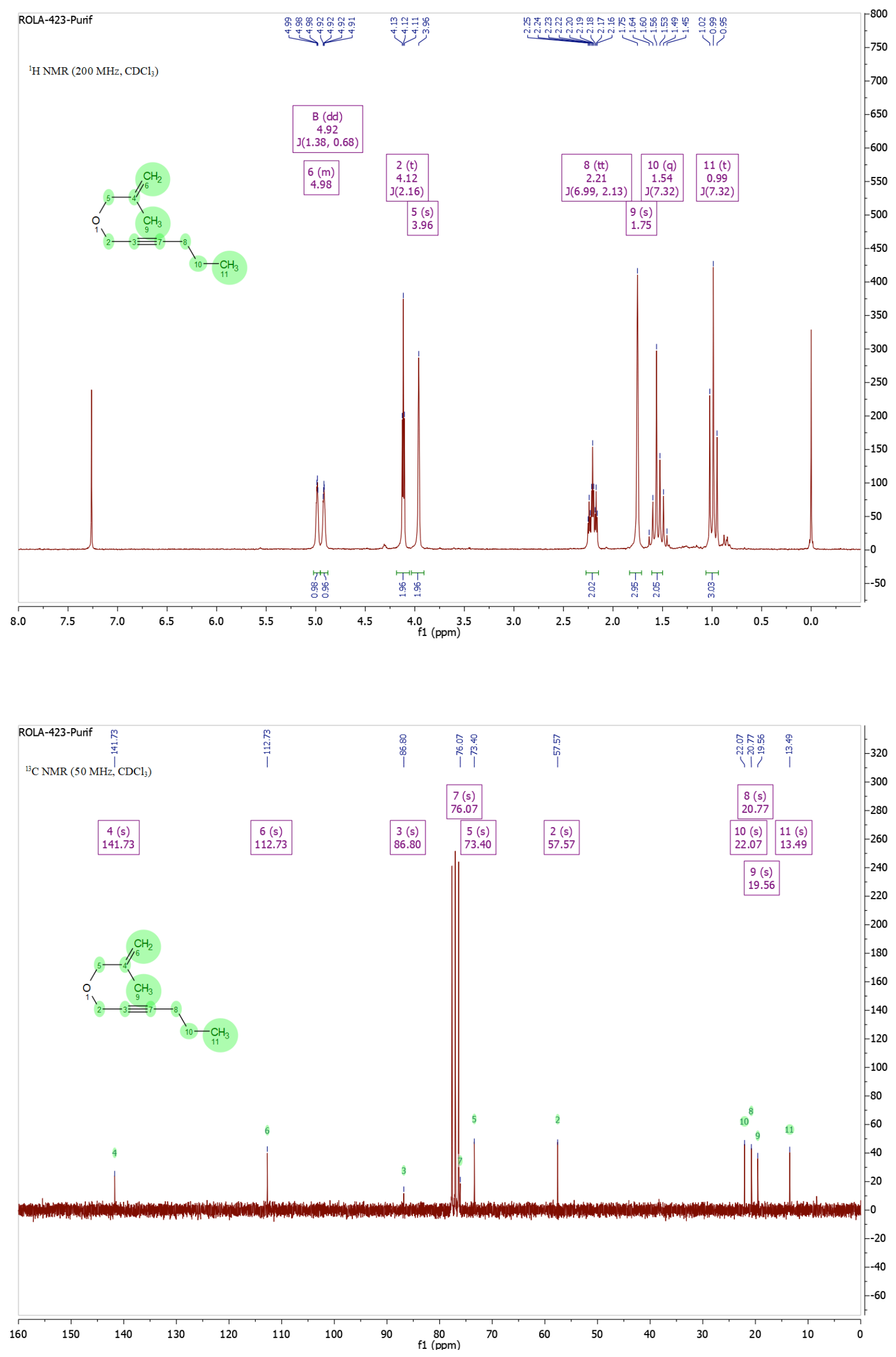
[1j]
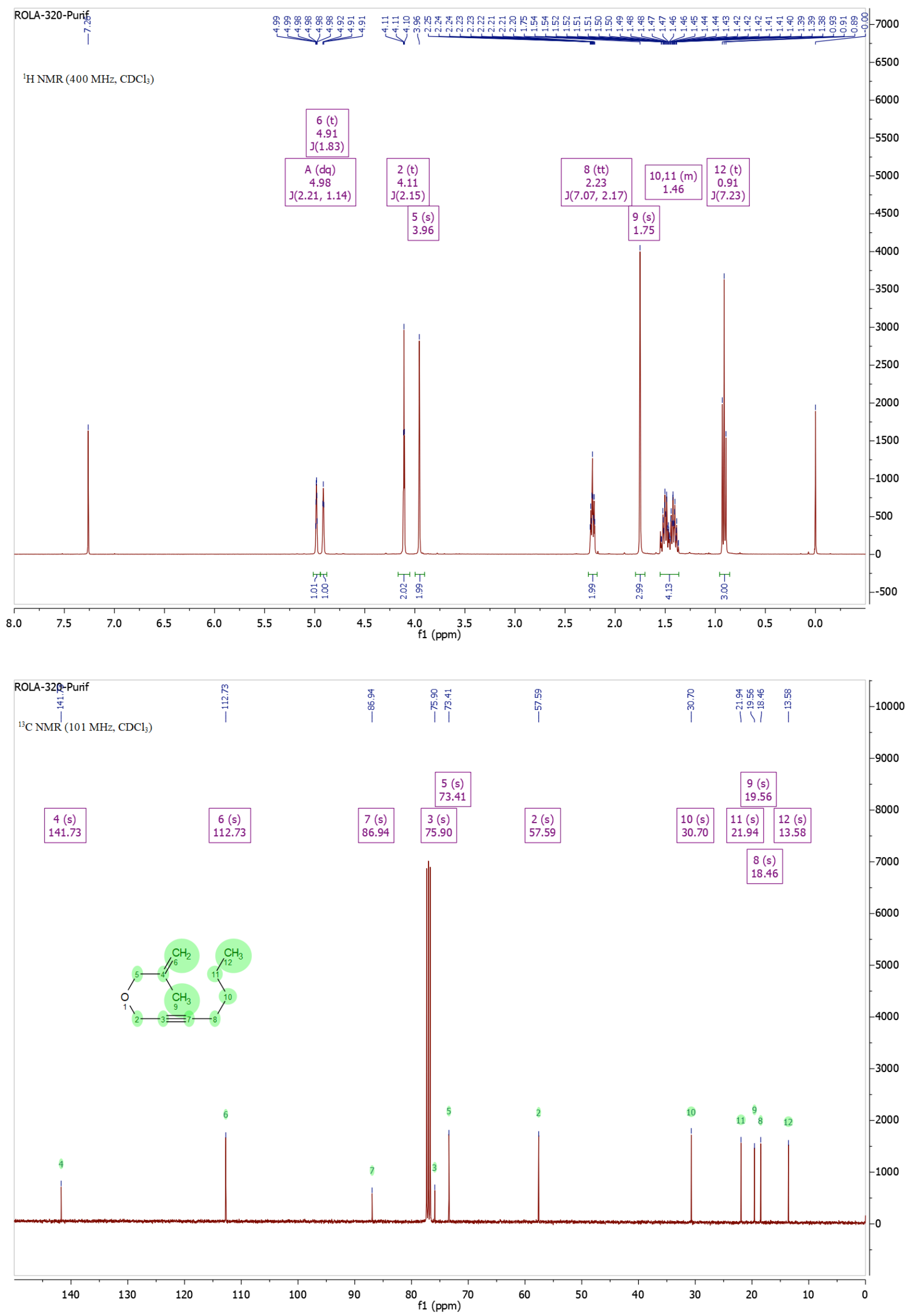
[1]]
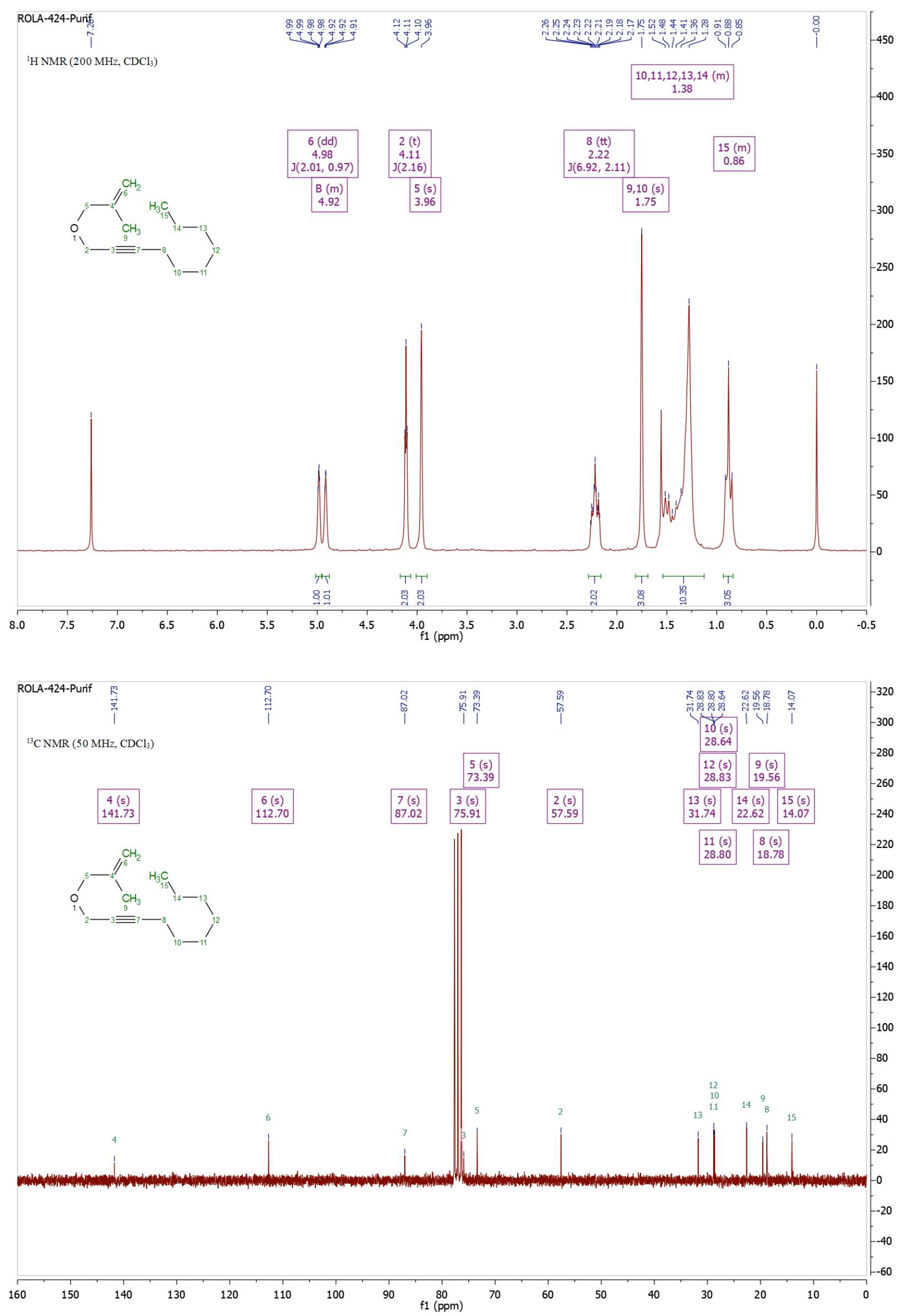
[1m]
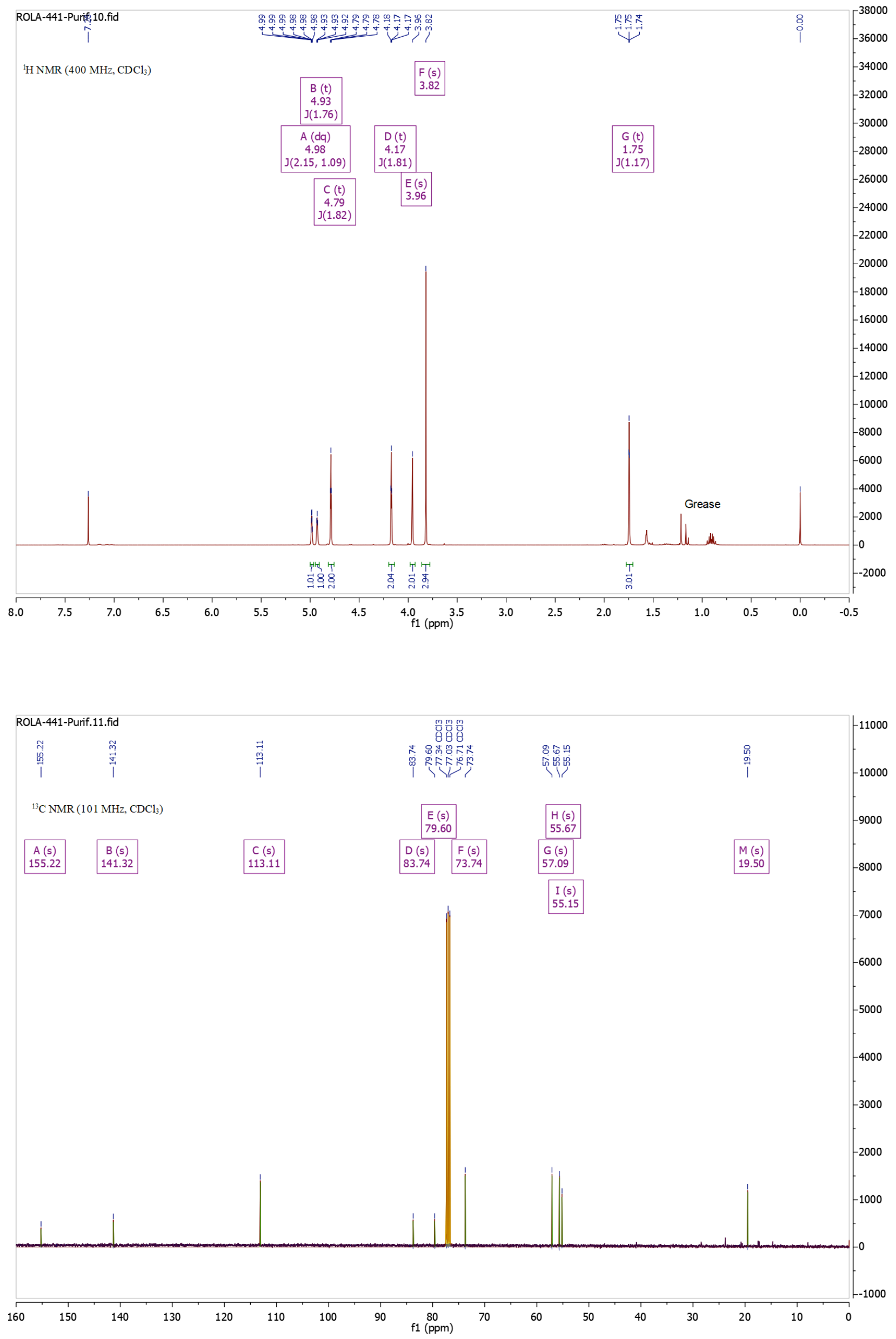
[10]
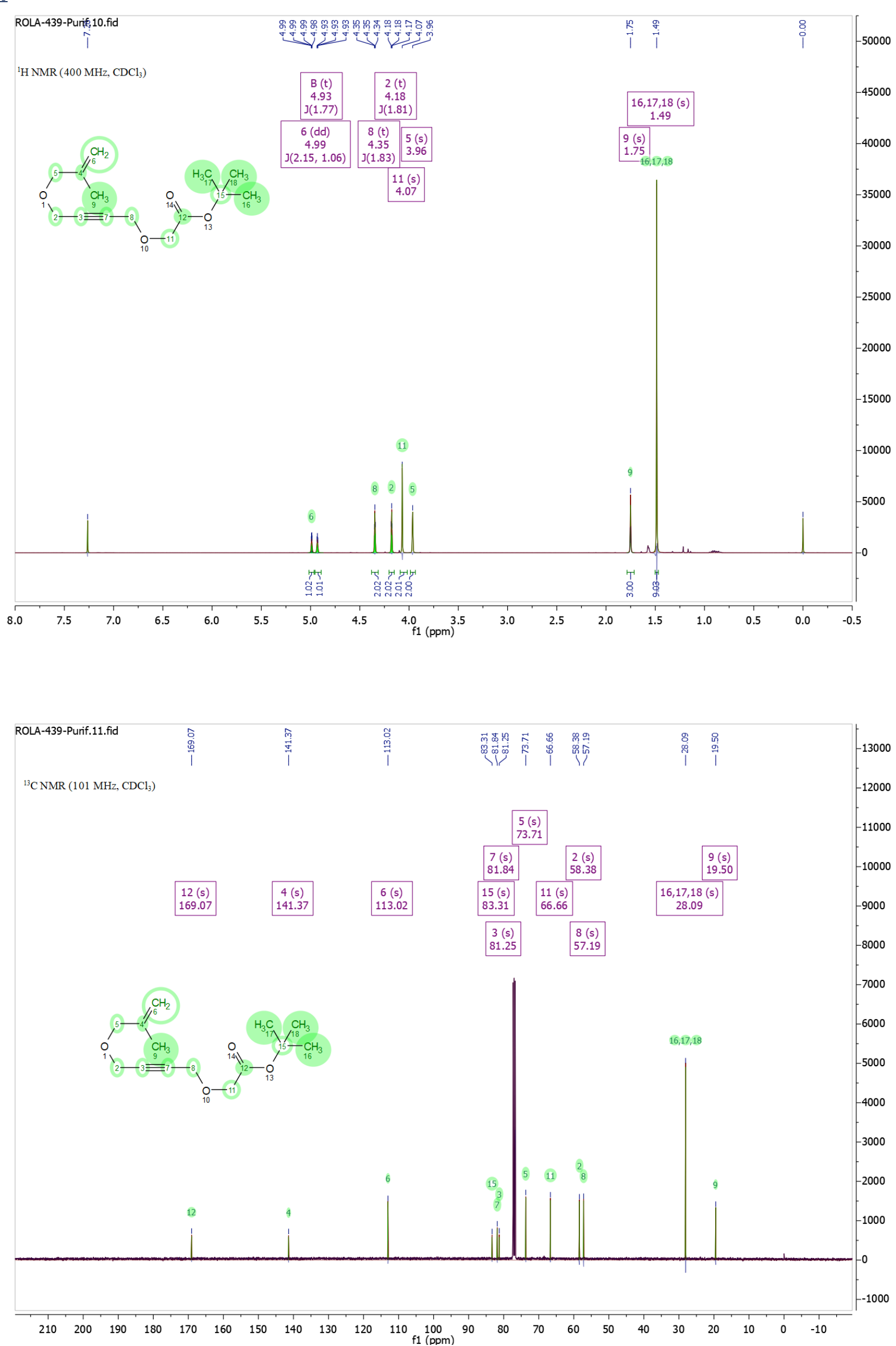
[1p]
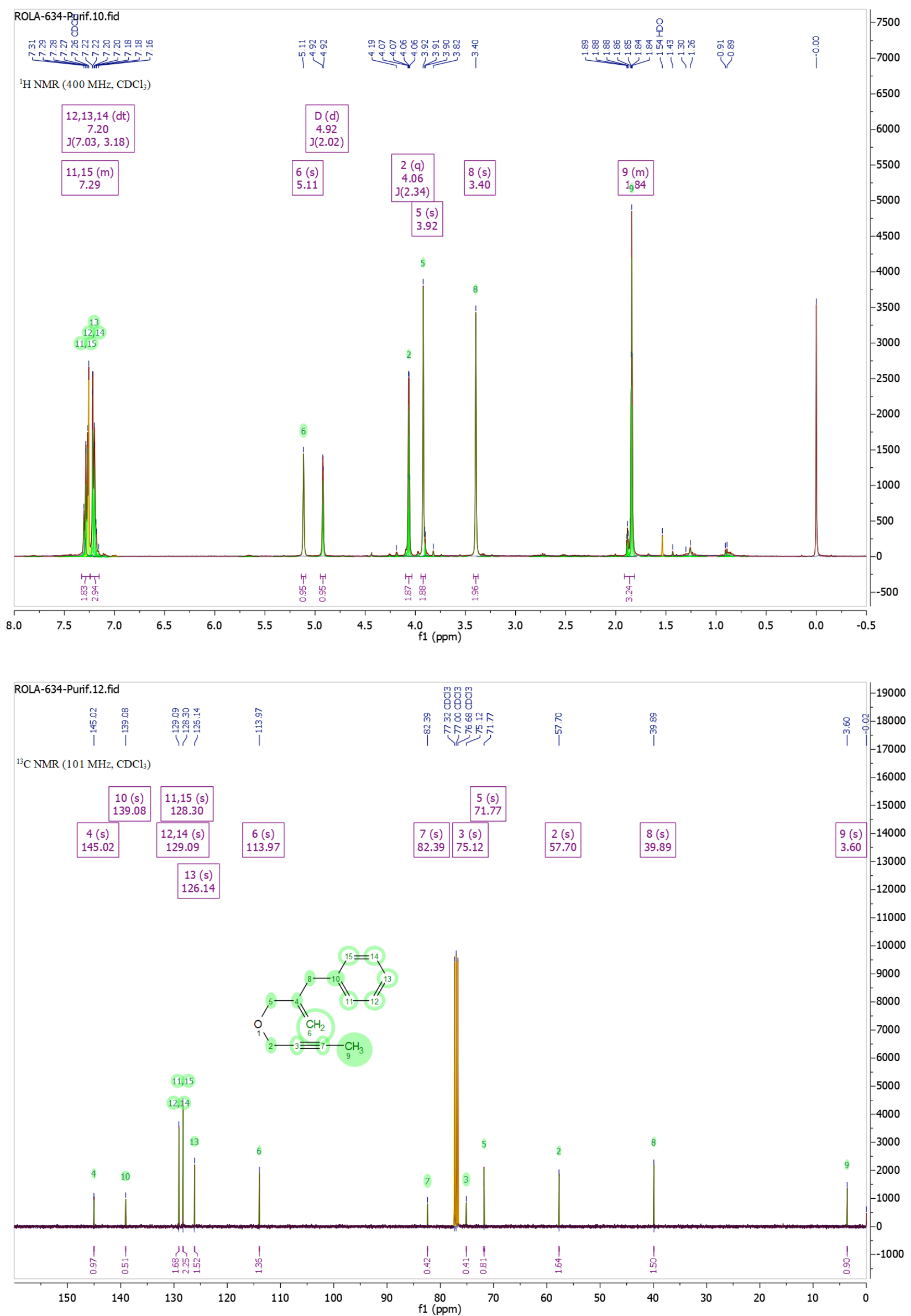
[1s]
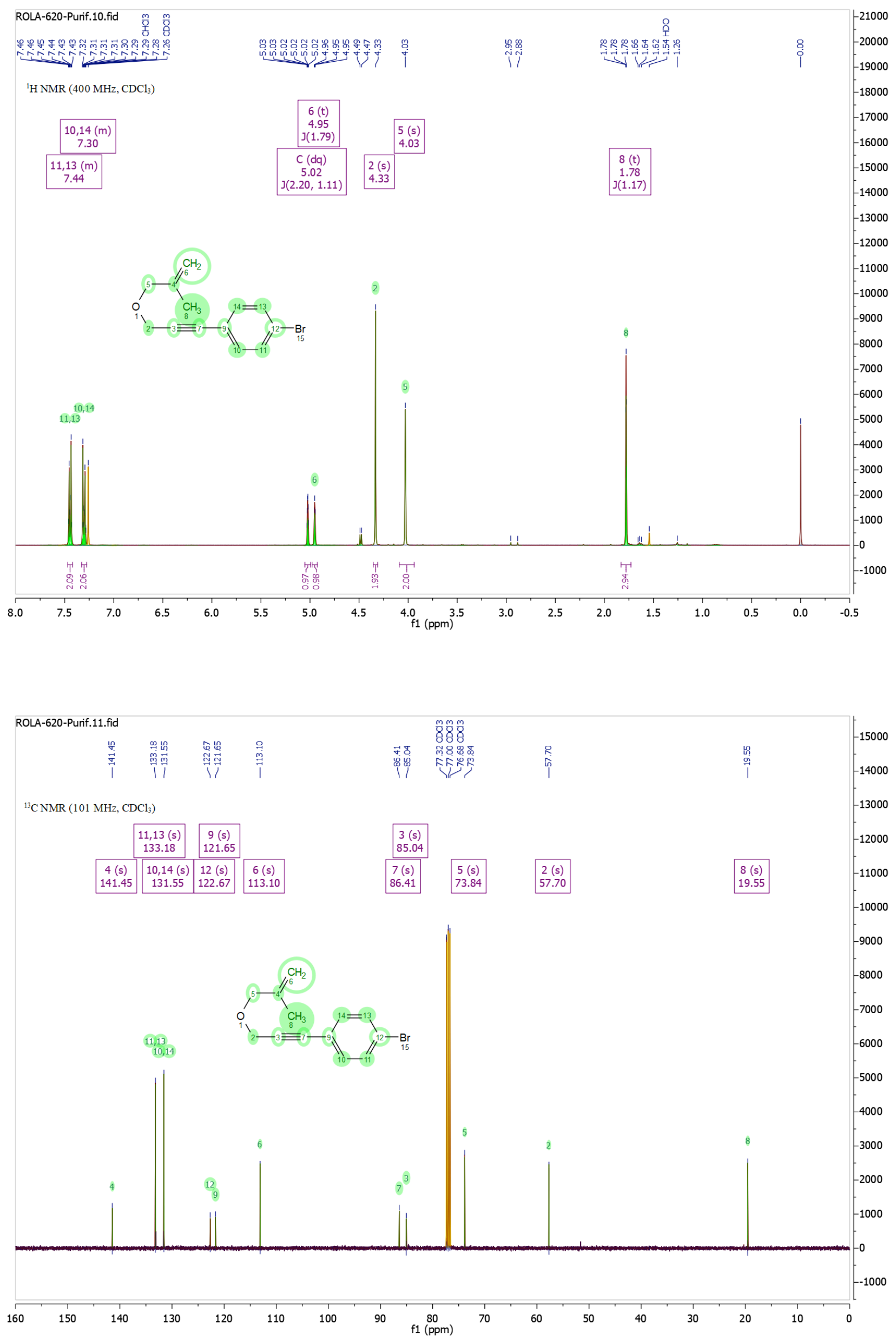
[1t]
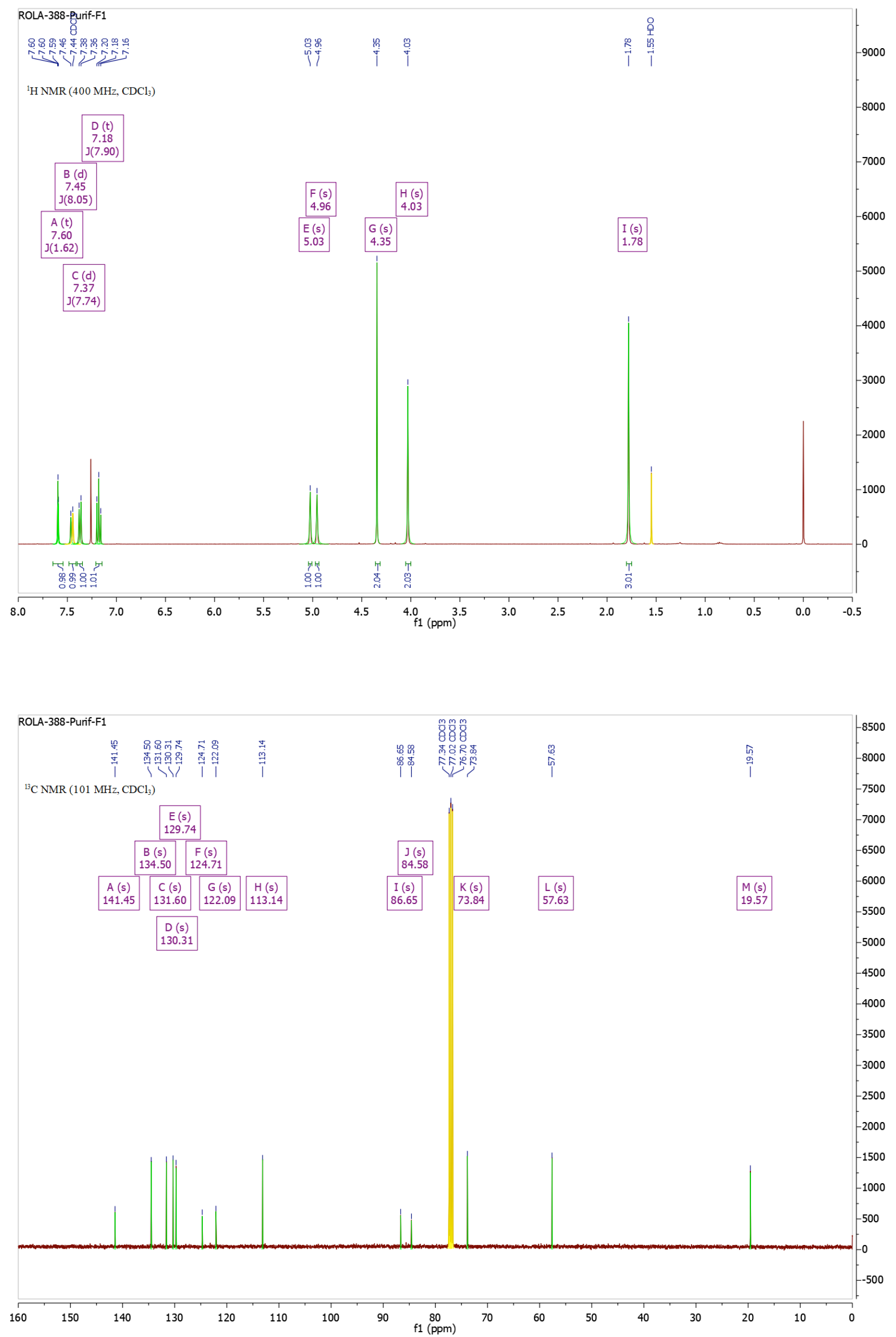
$\underline{[1 \mathrm{v}]}$
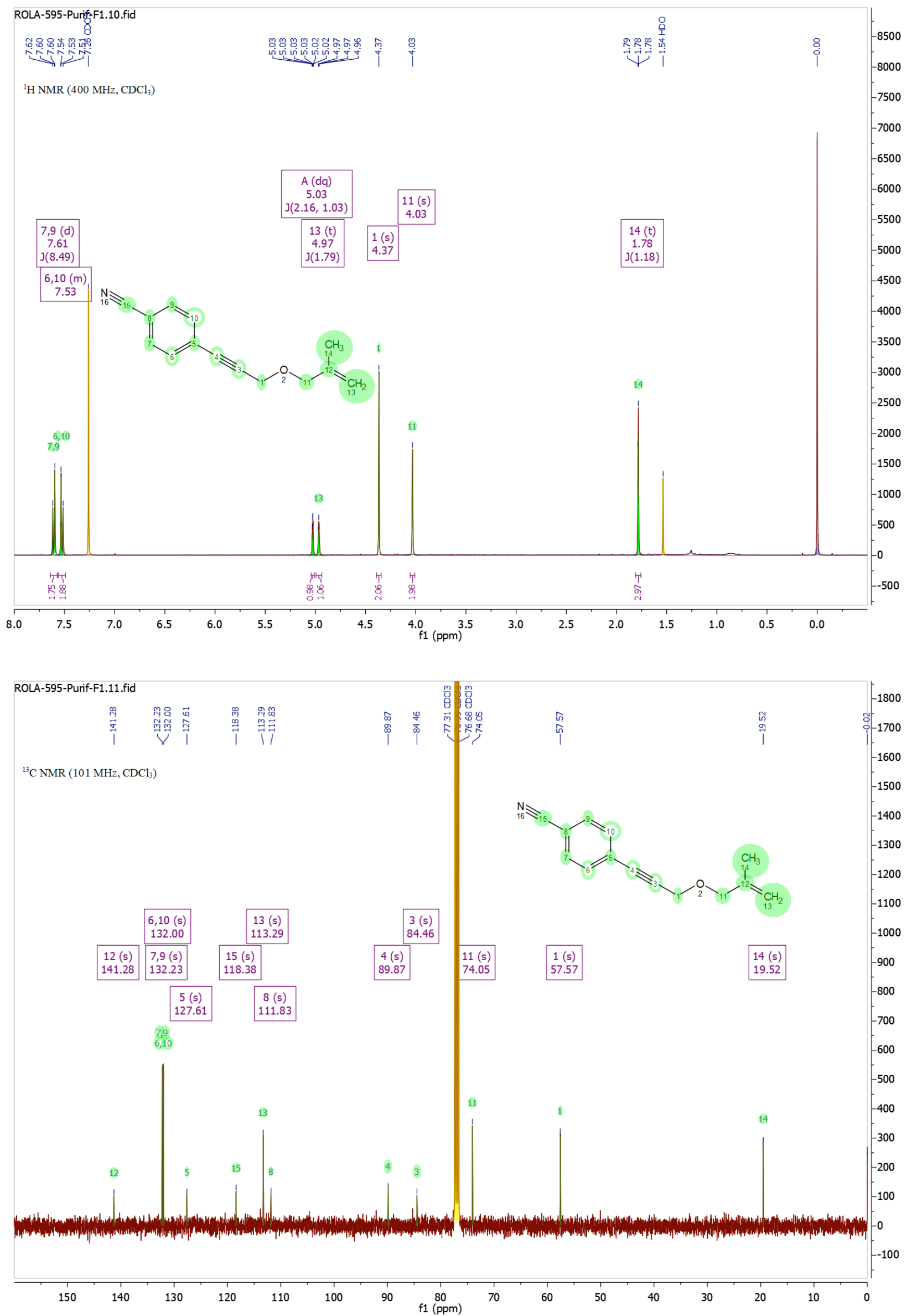
2. NMR spectra of bicyclic compounds

$\underline{[2 a]}$
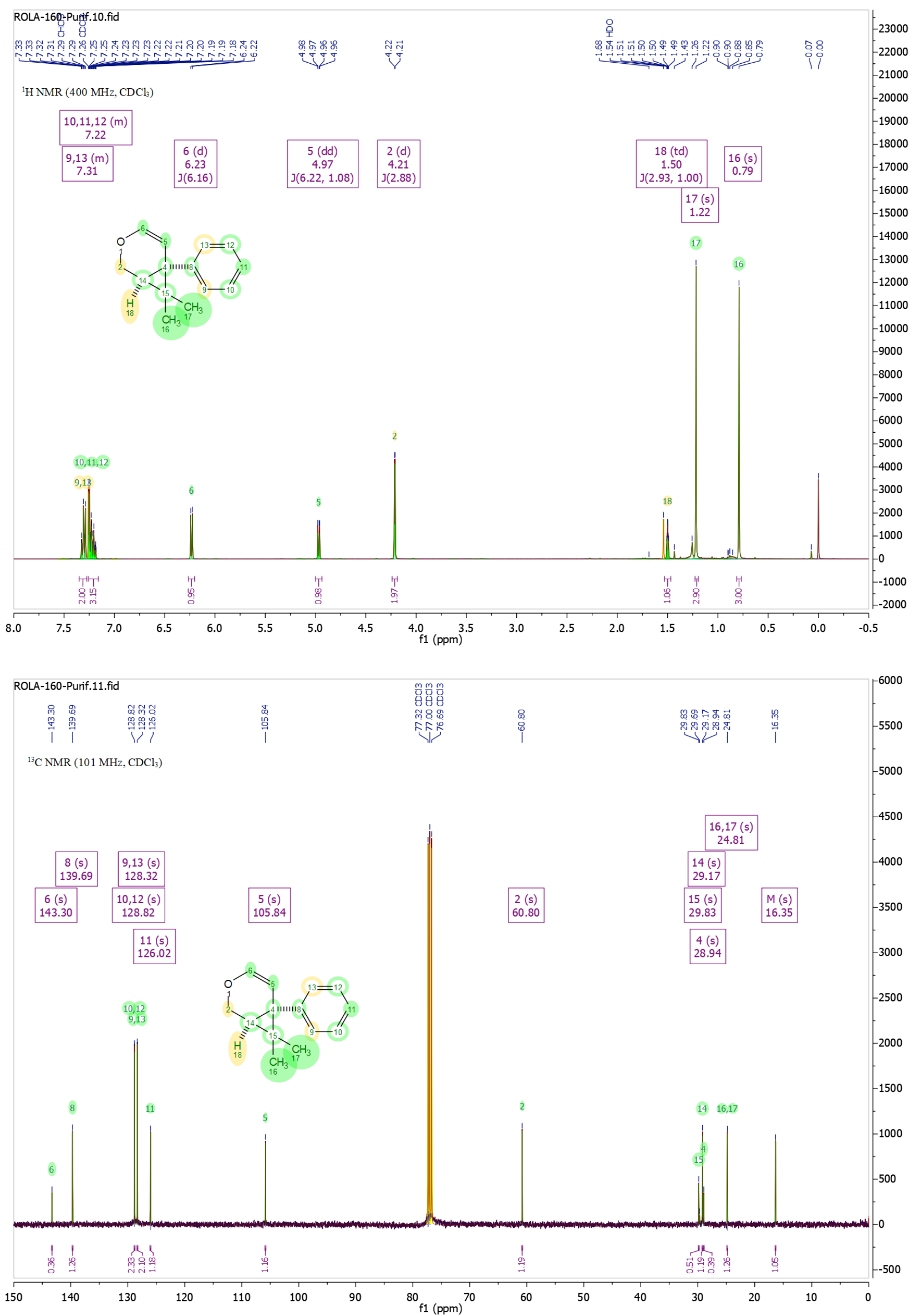
[2e]
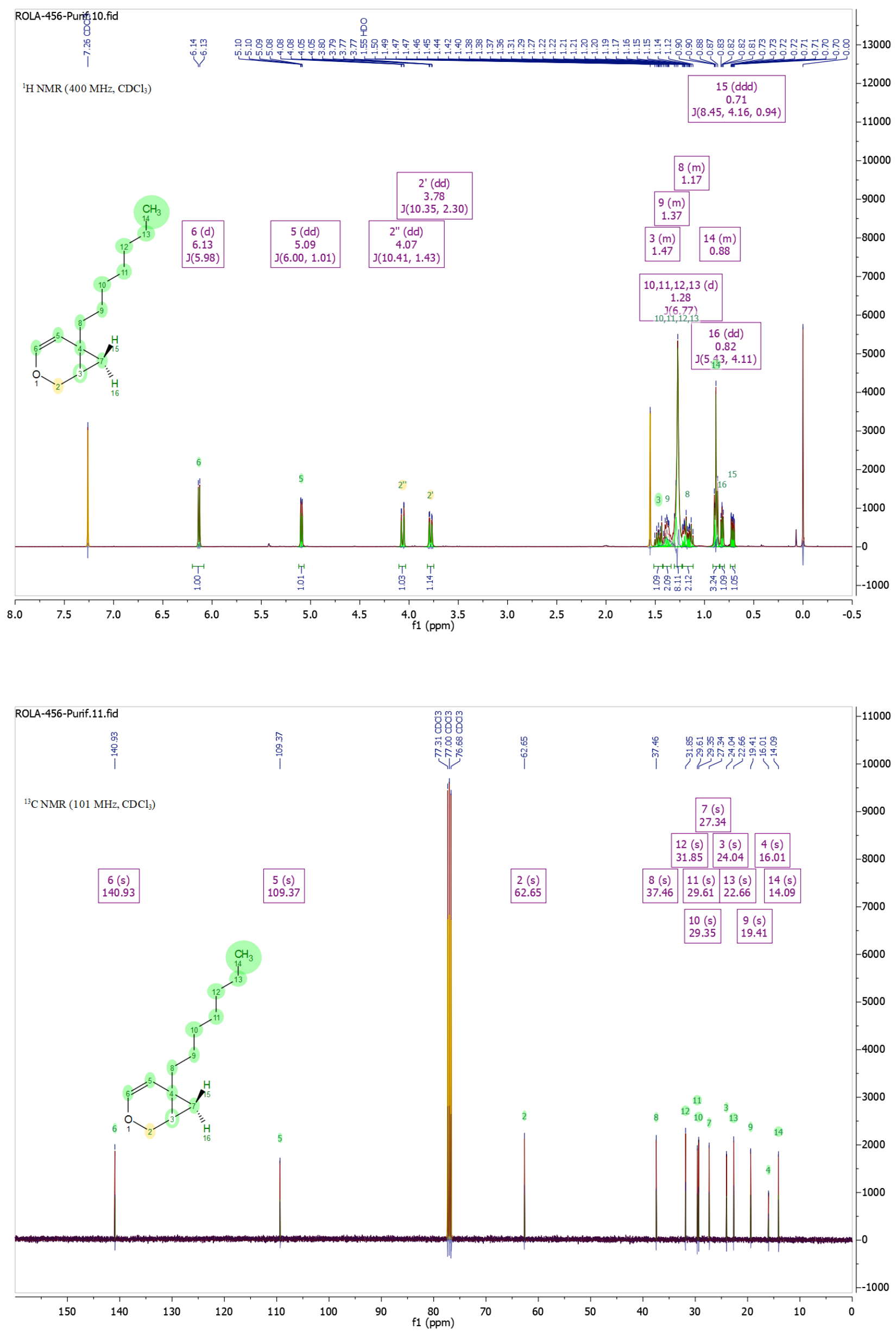
[2h]
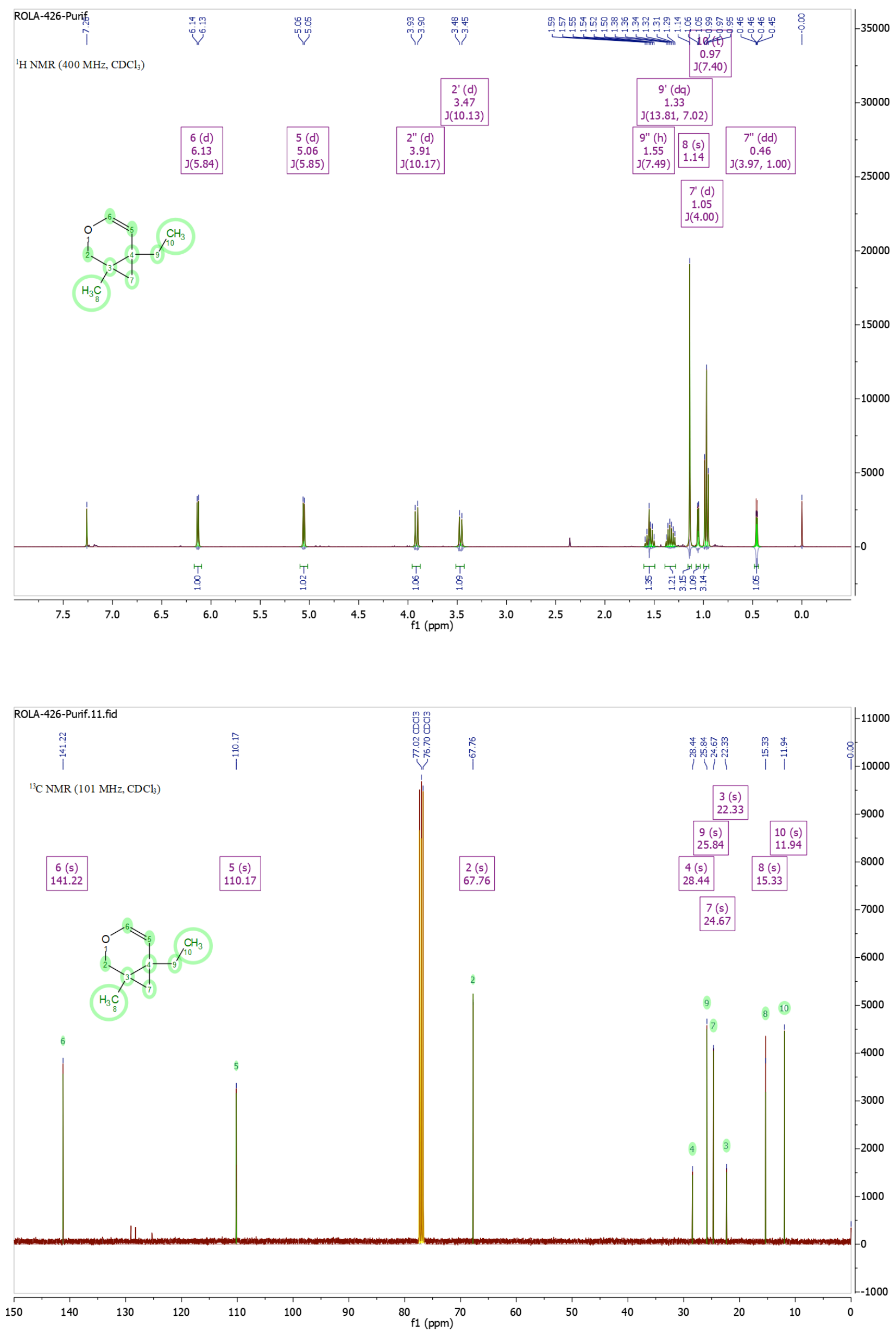
[2i]
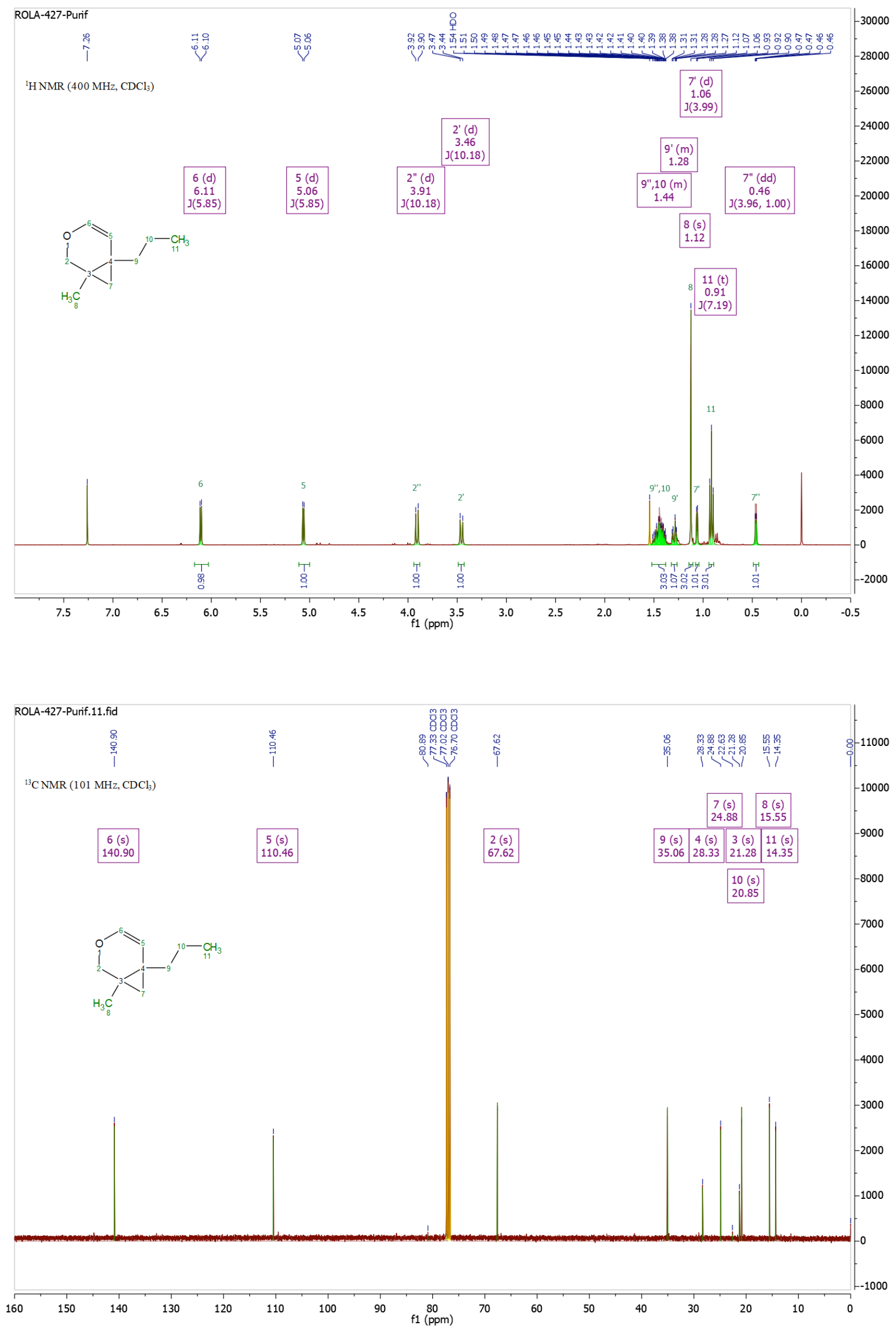
[2j]
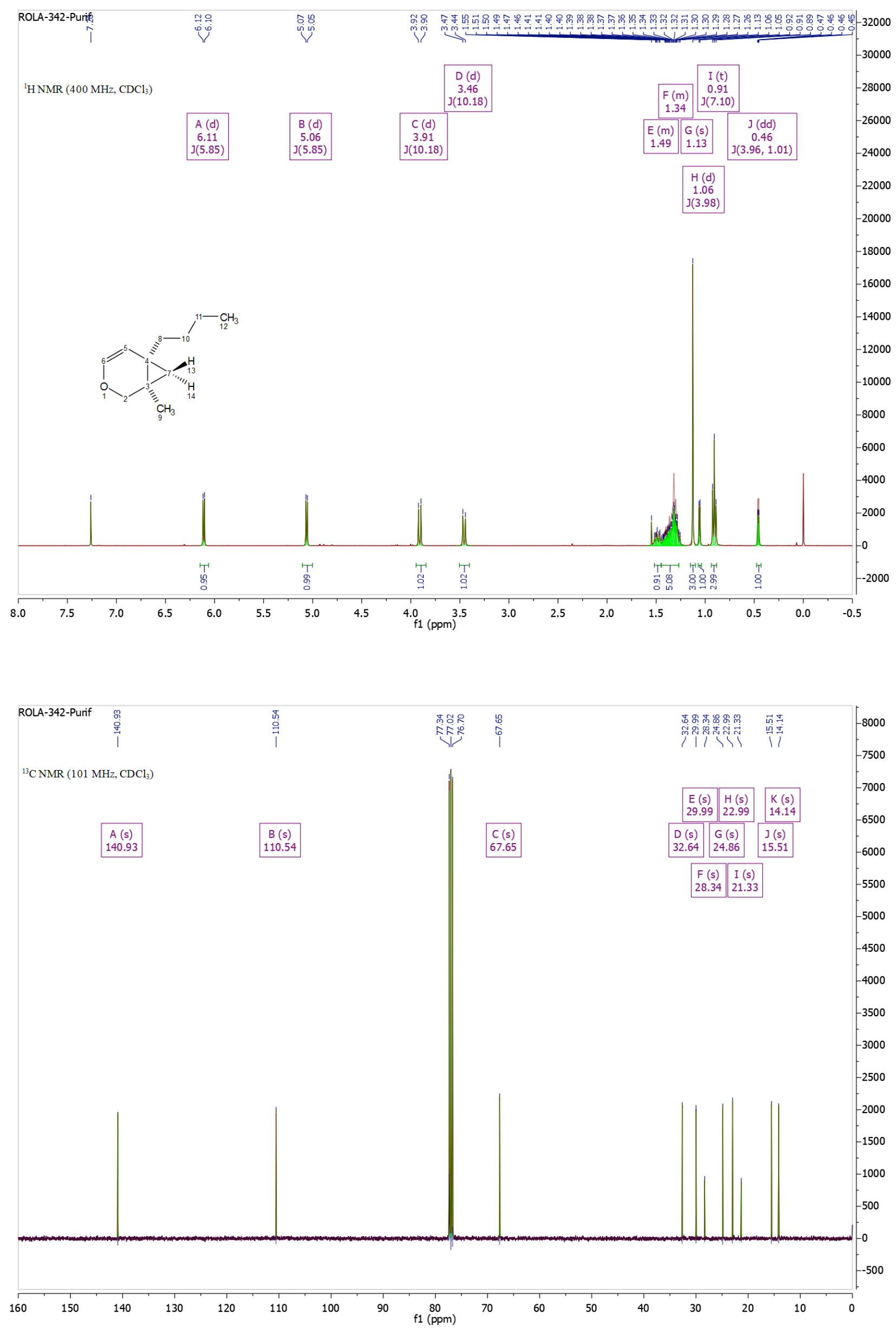
[2k]
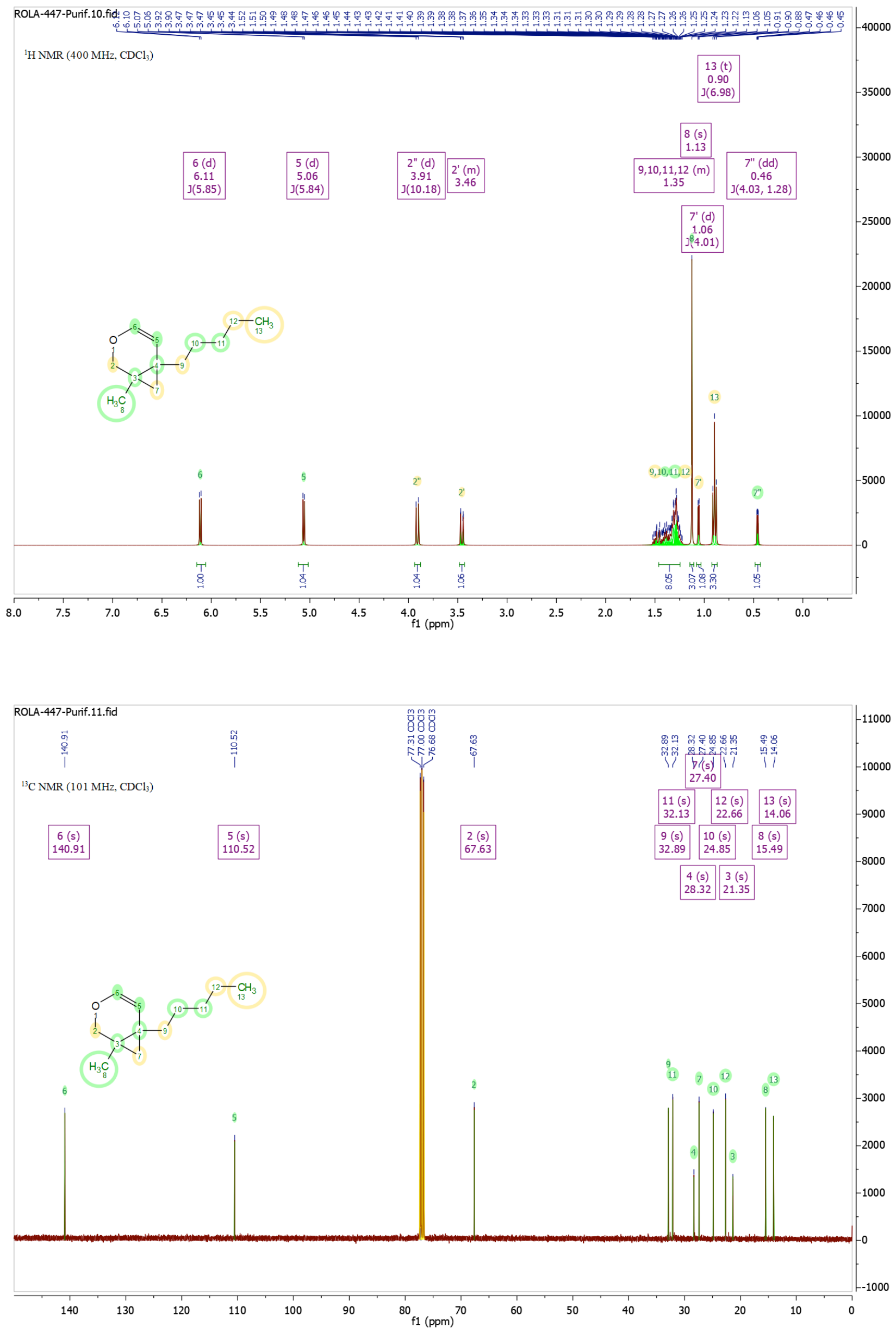
[2I]
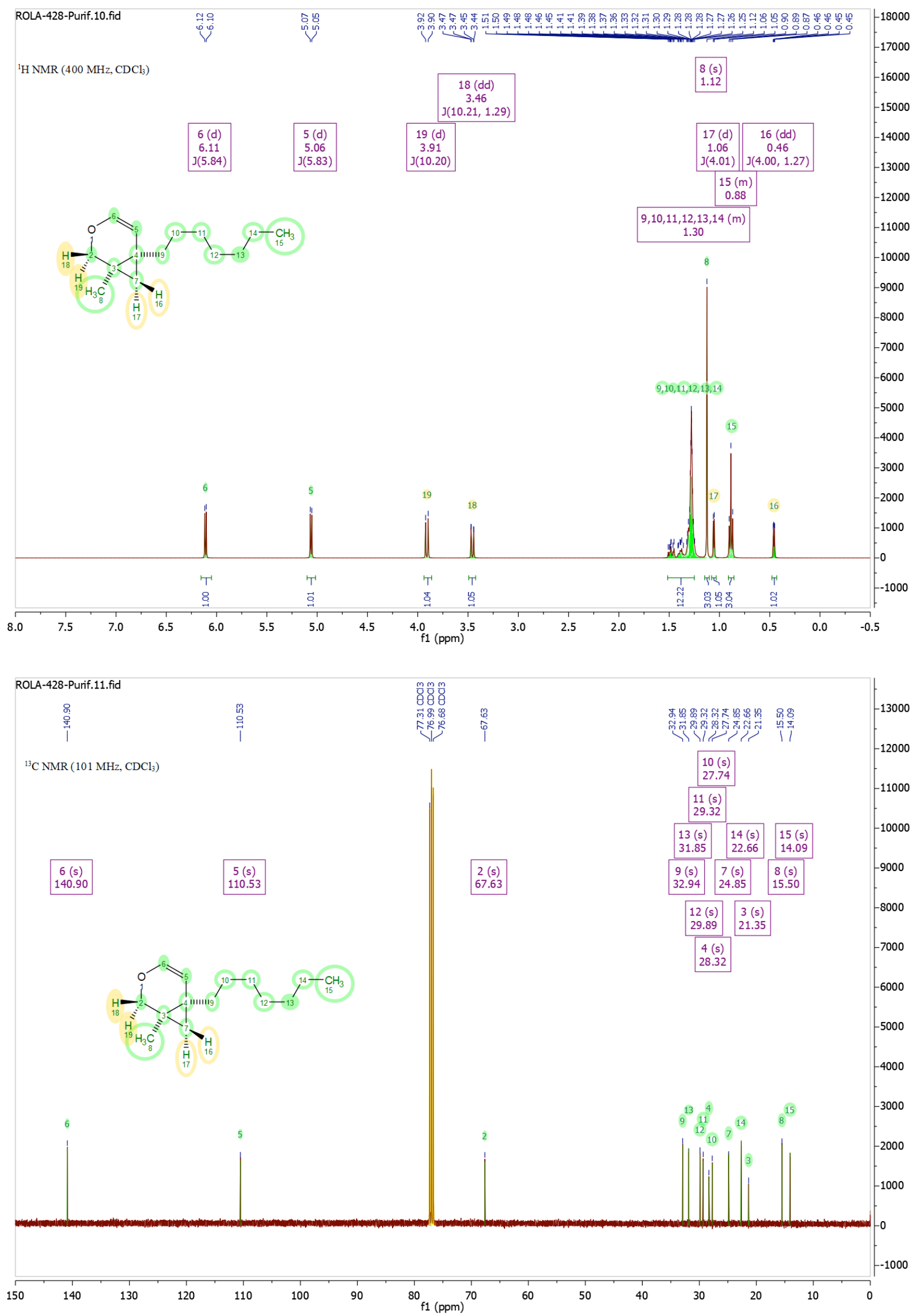
[2m]
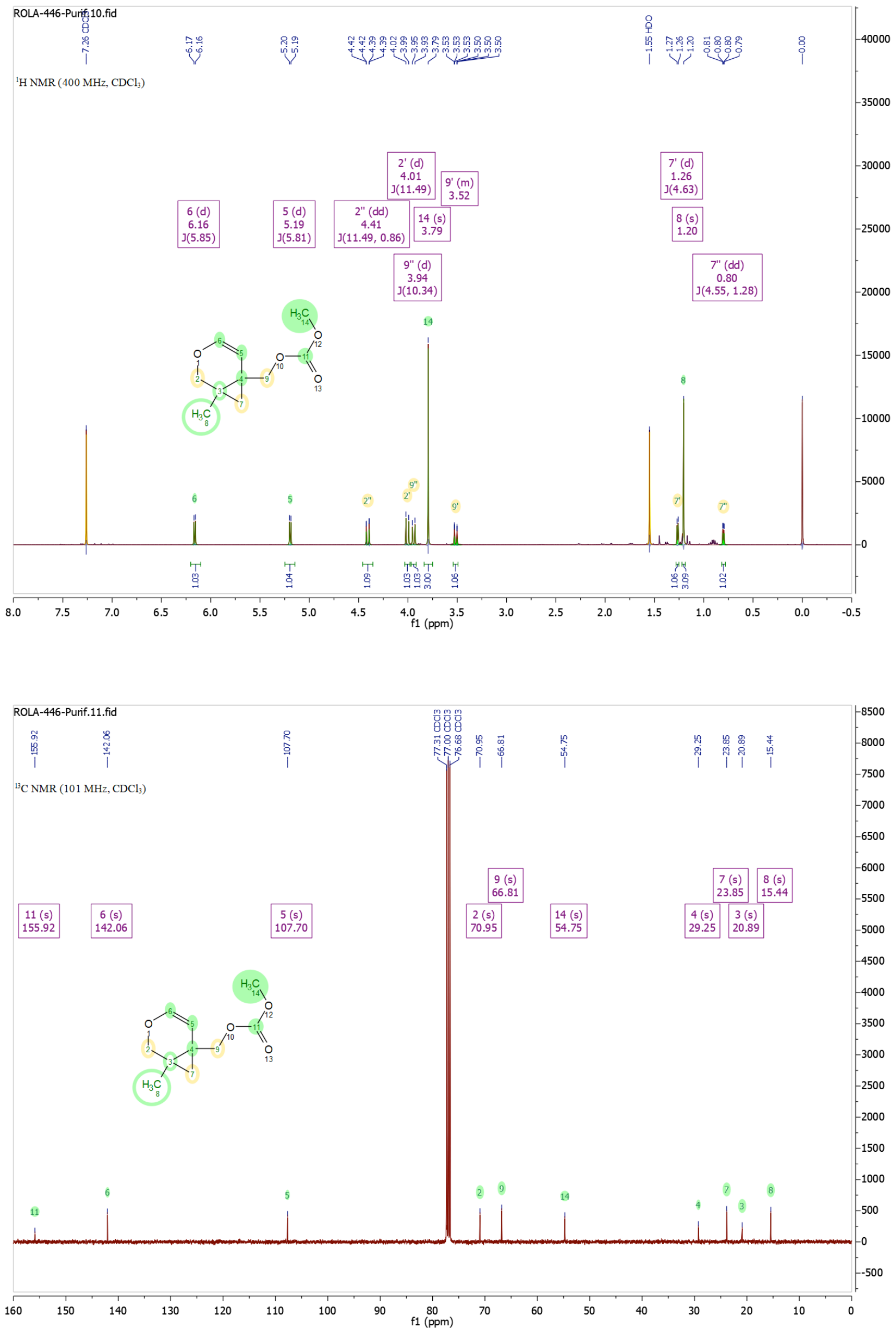
[2n]
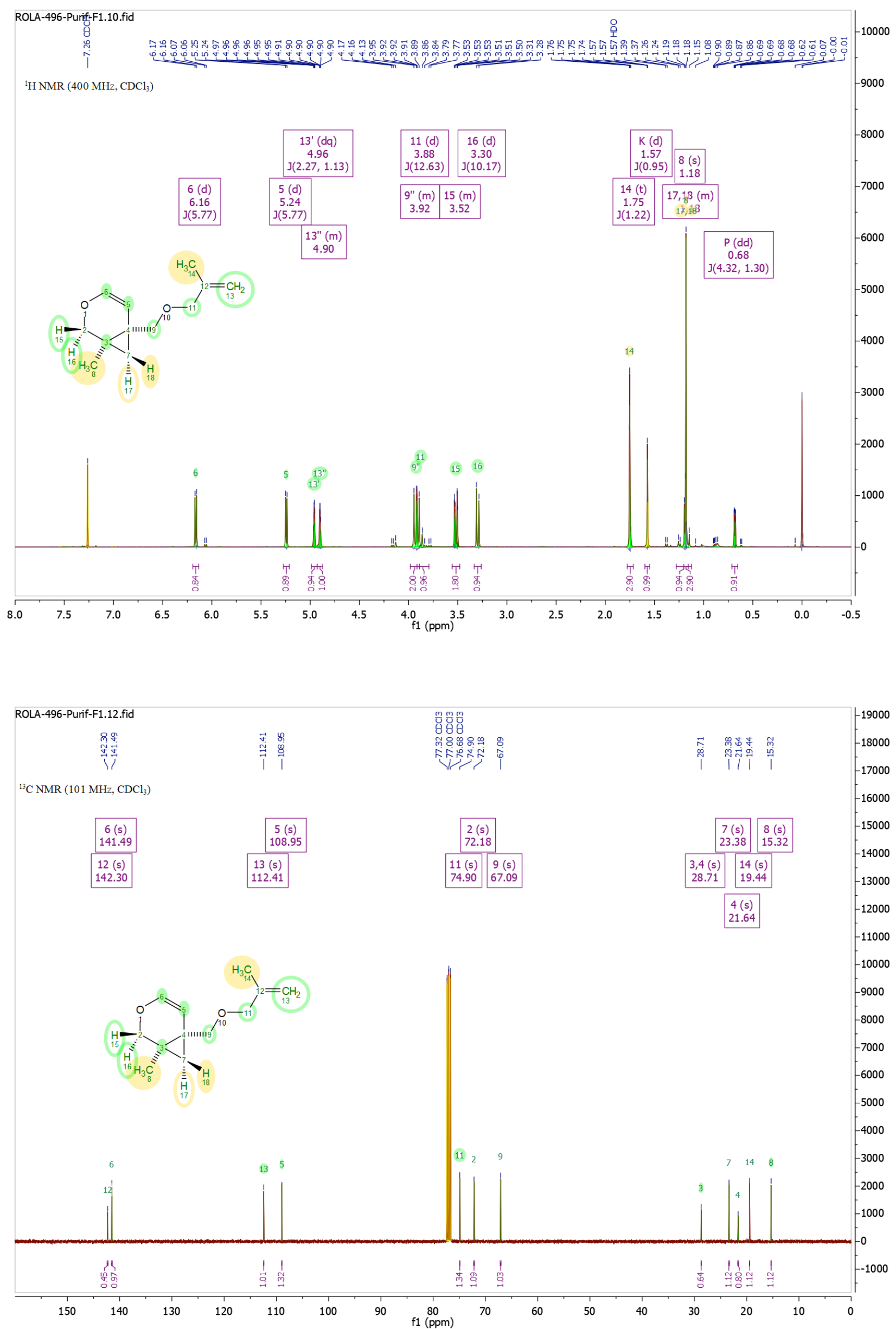

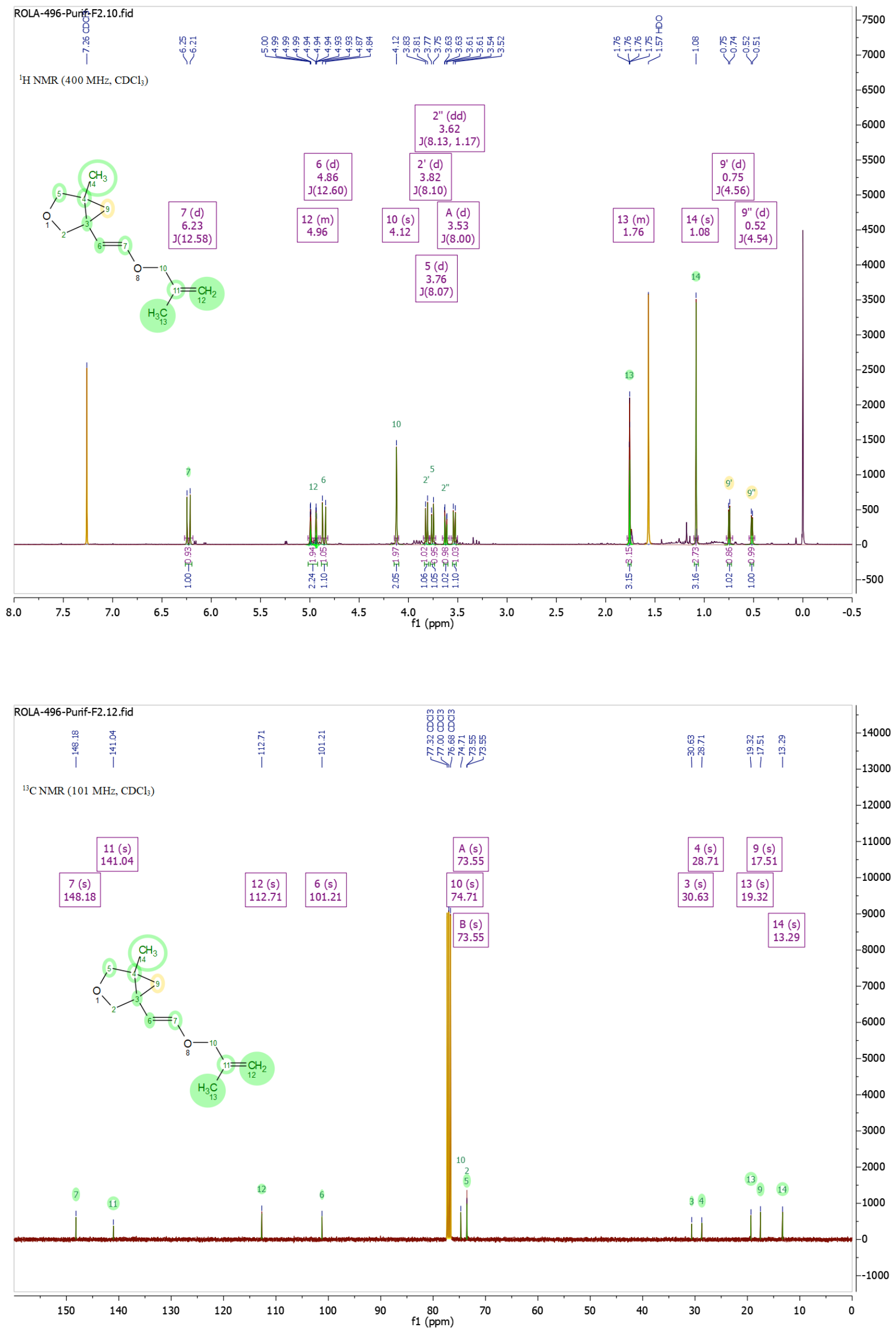
[20]
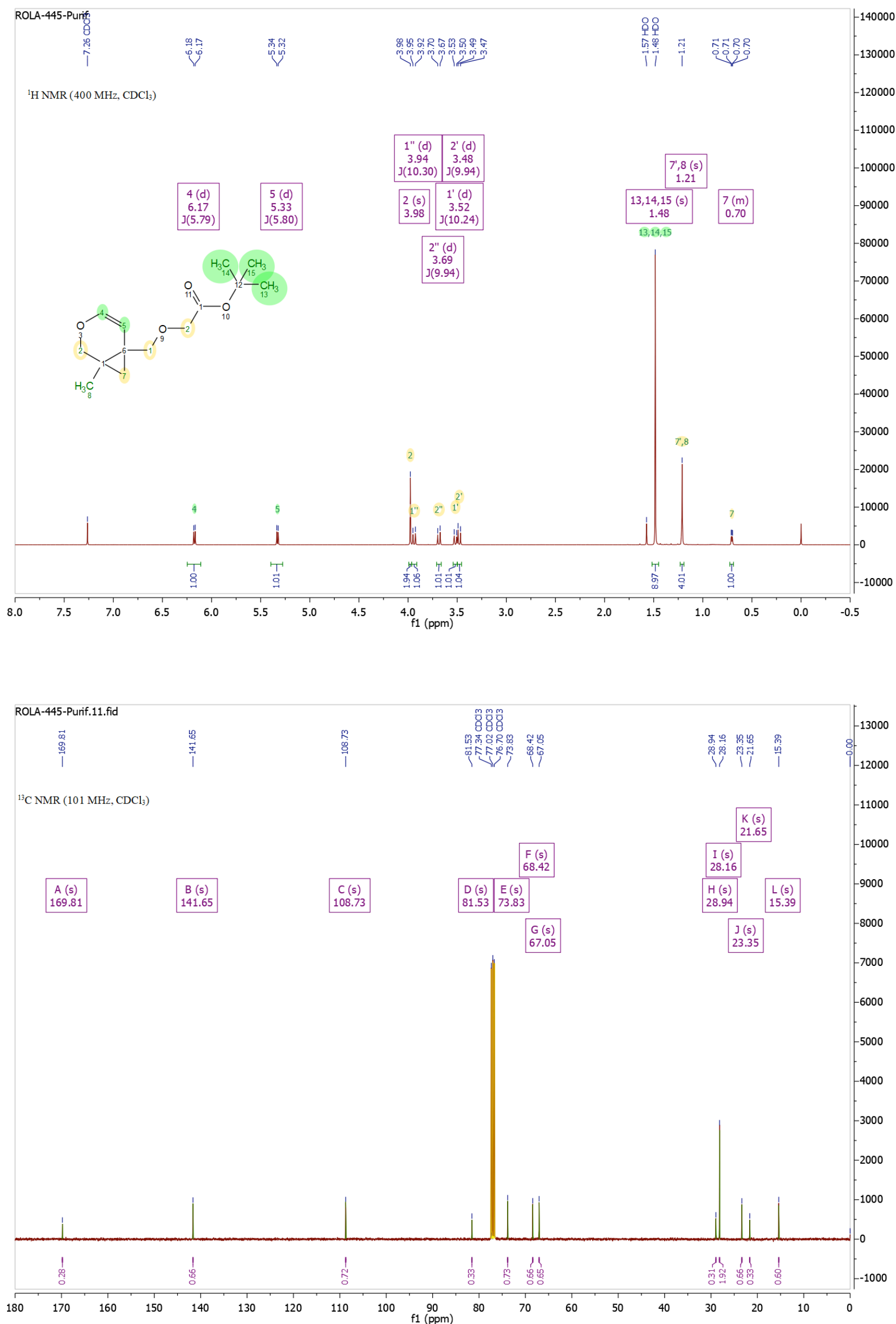
[2p]

ROLA-639-Brut.10.fid $\frac{m}{\mathrm{Z}} \frac{m}{\mathrm{O}}$

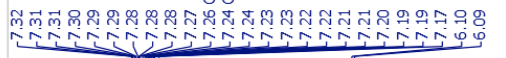

${ }^{1} \mathrm{H} \mathrm{NMR}\left(400 \mathrm{MHz}, \mathrm{CDCl}_{3}\right)$

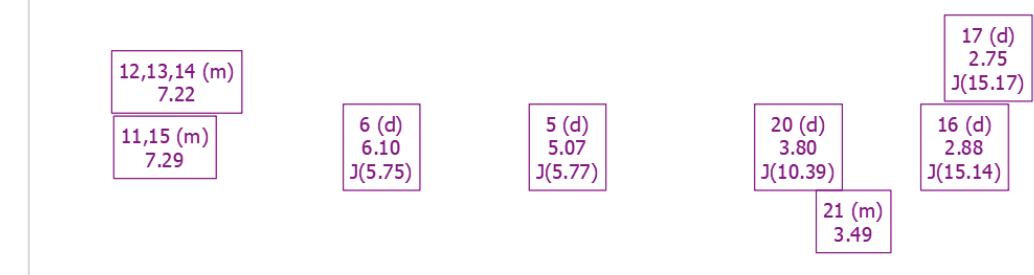

นุำ

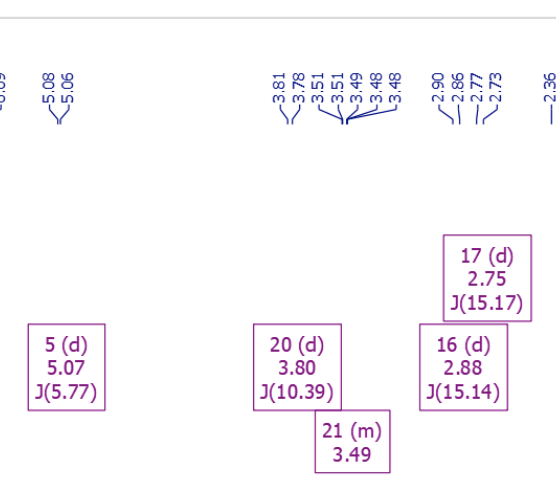

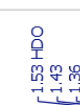

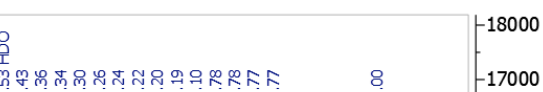

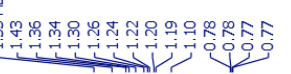

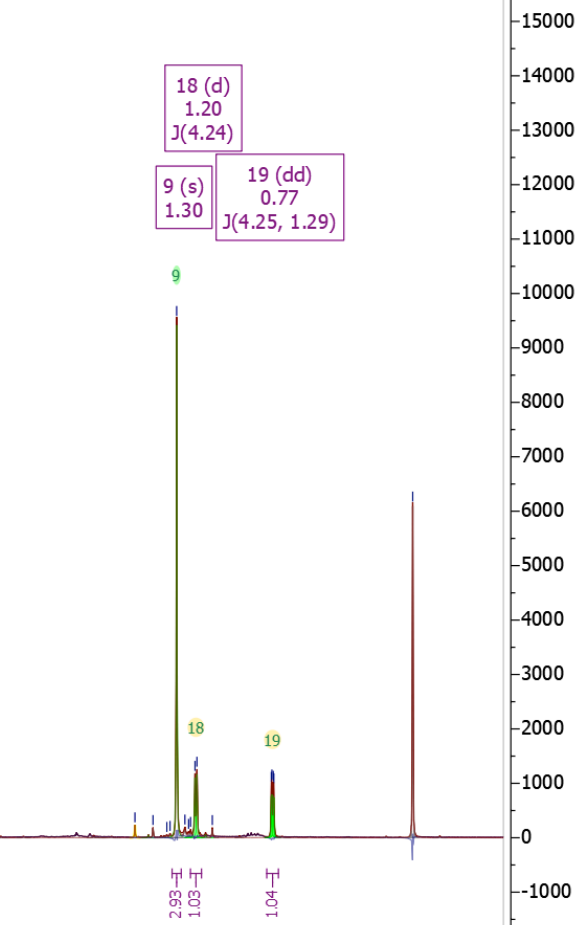

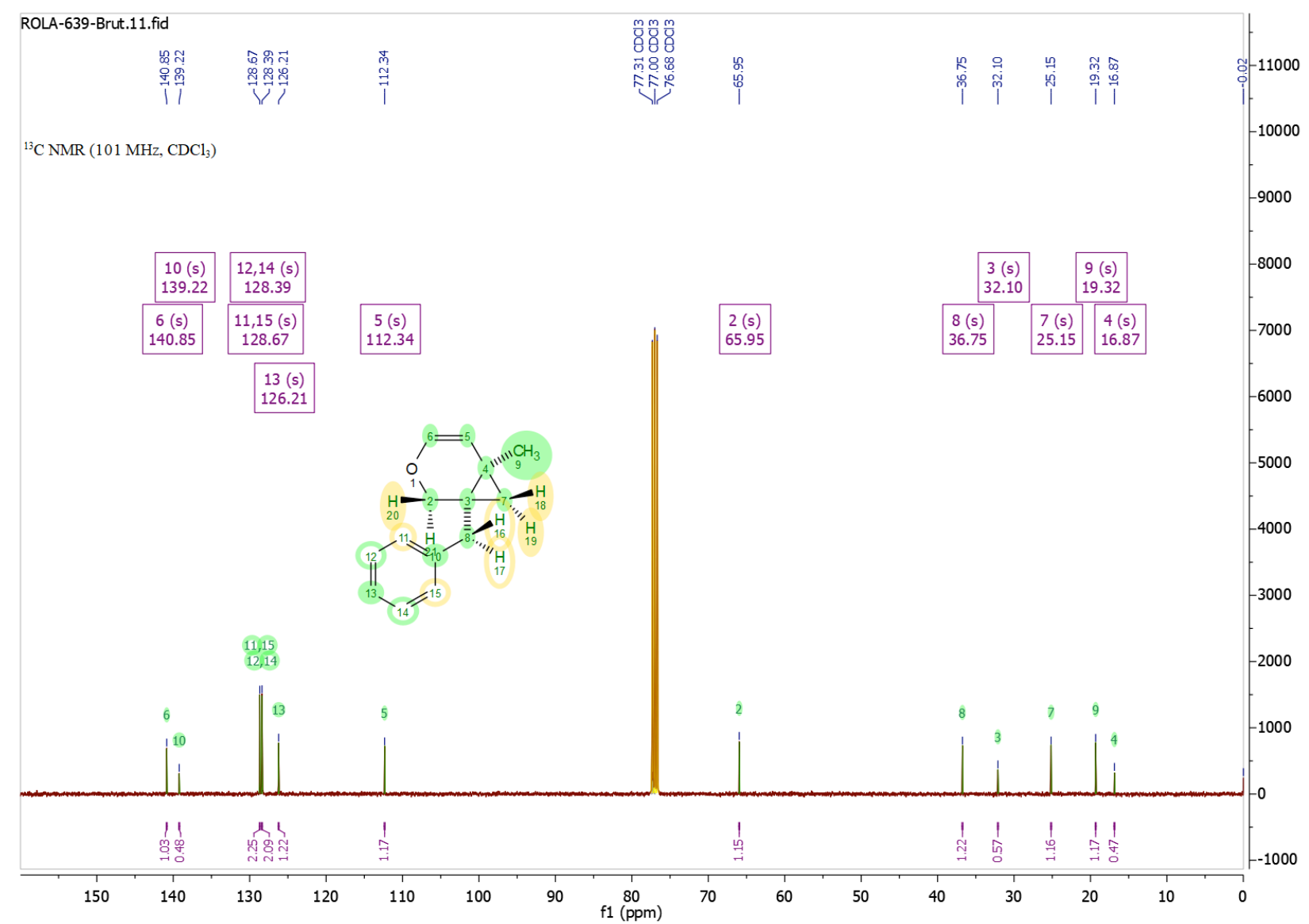


[2q]
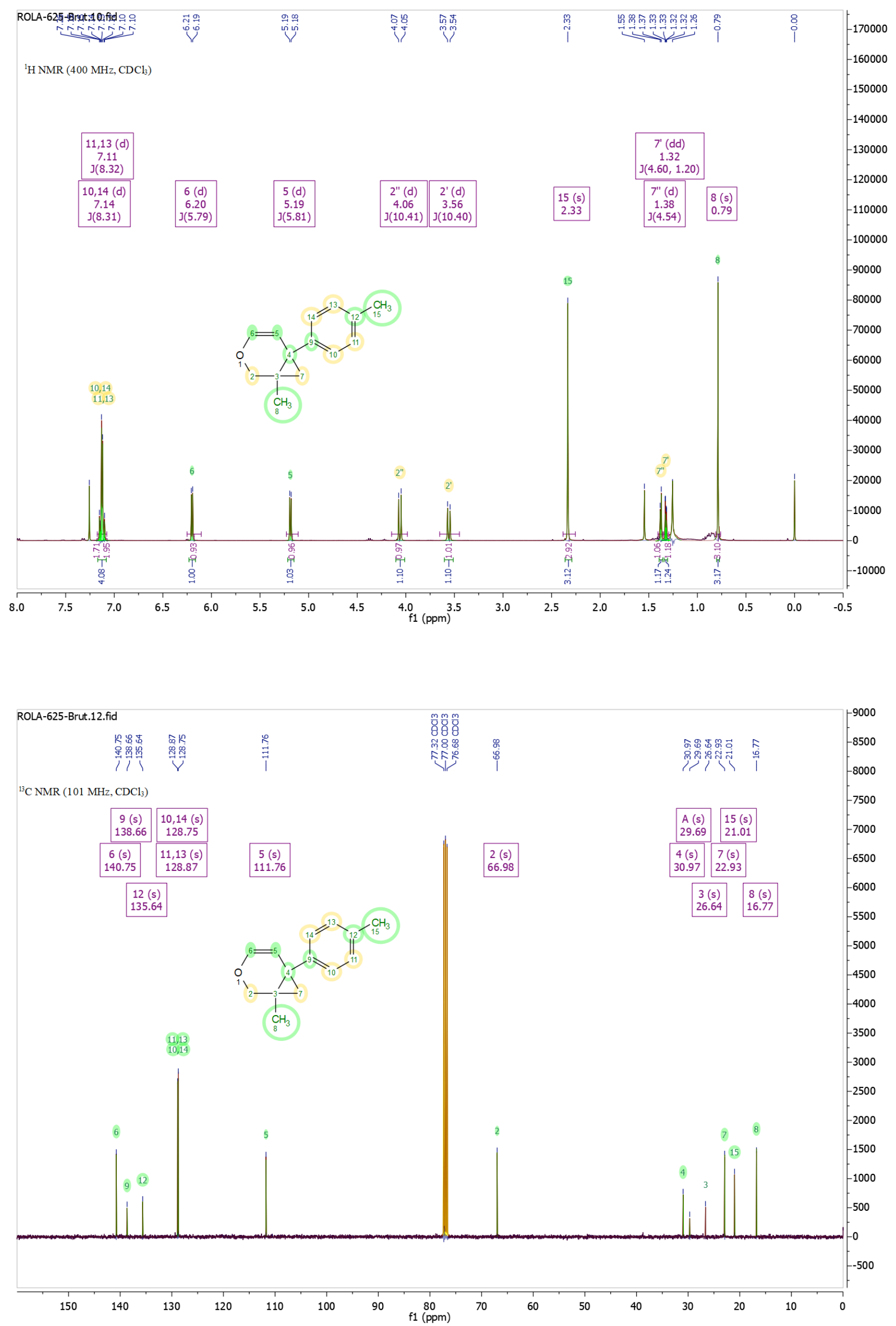
[2r]
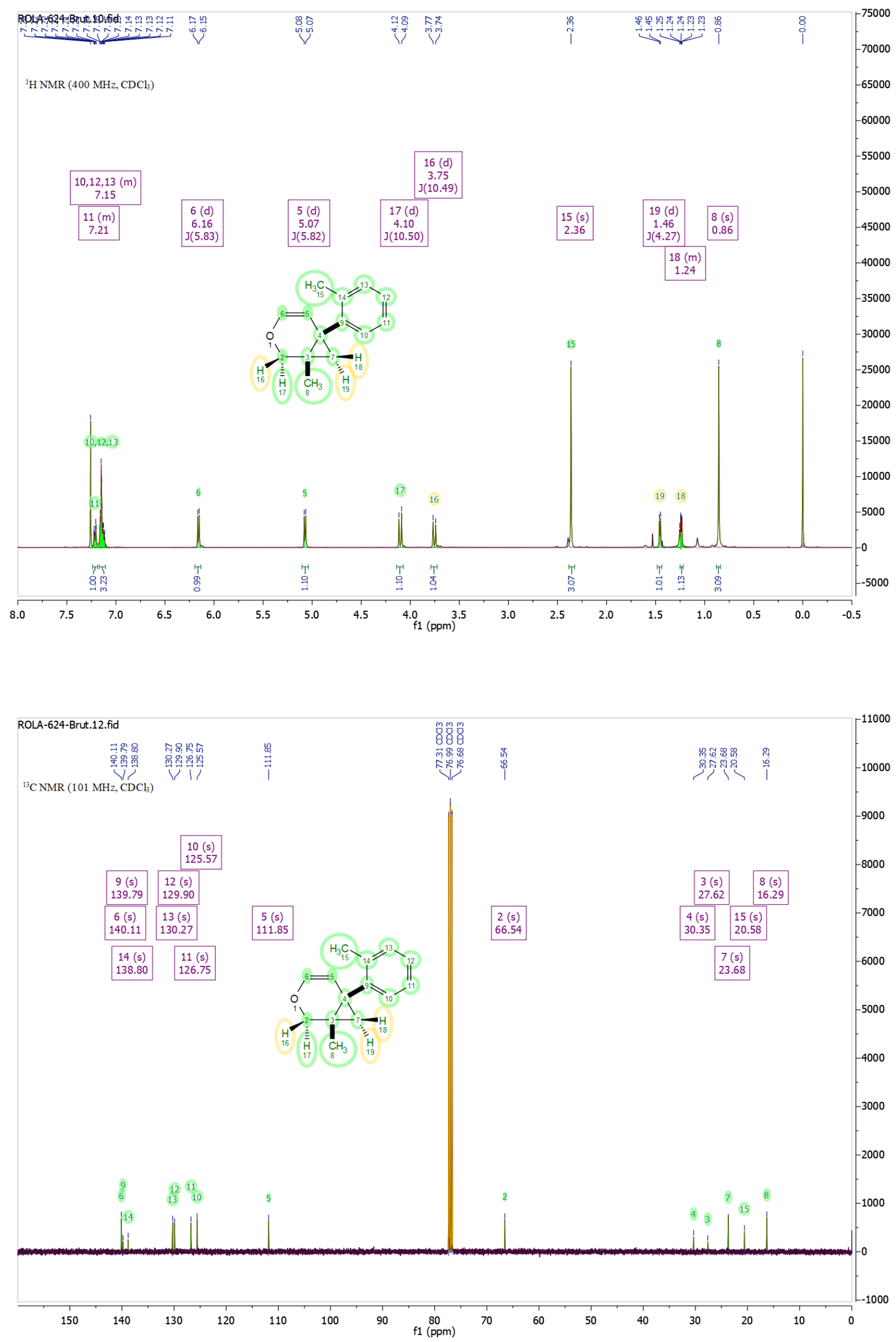
[2s]
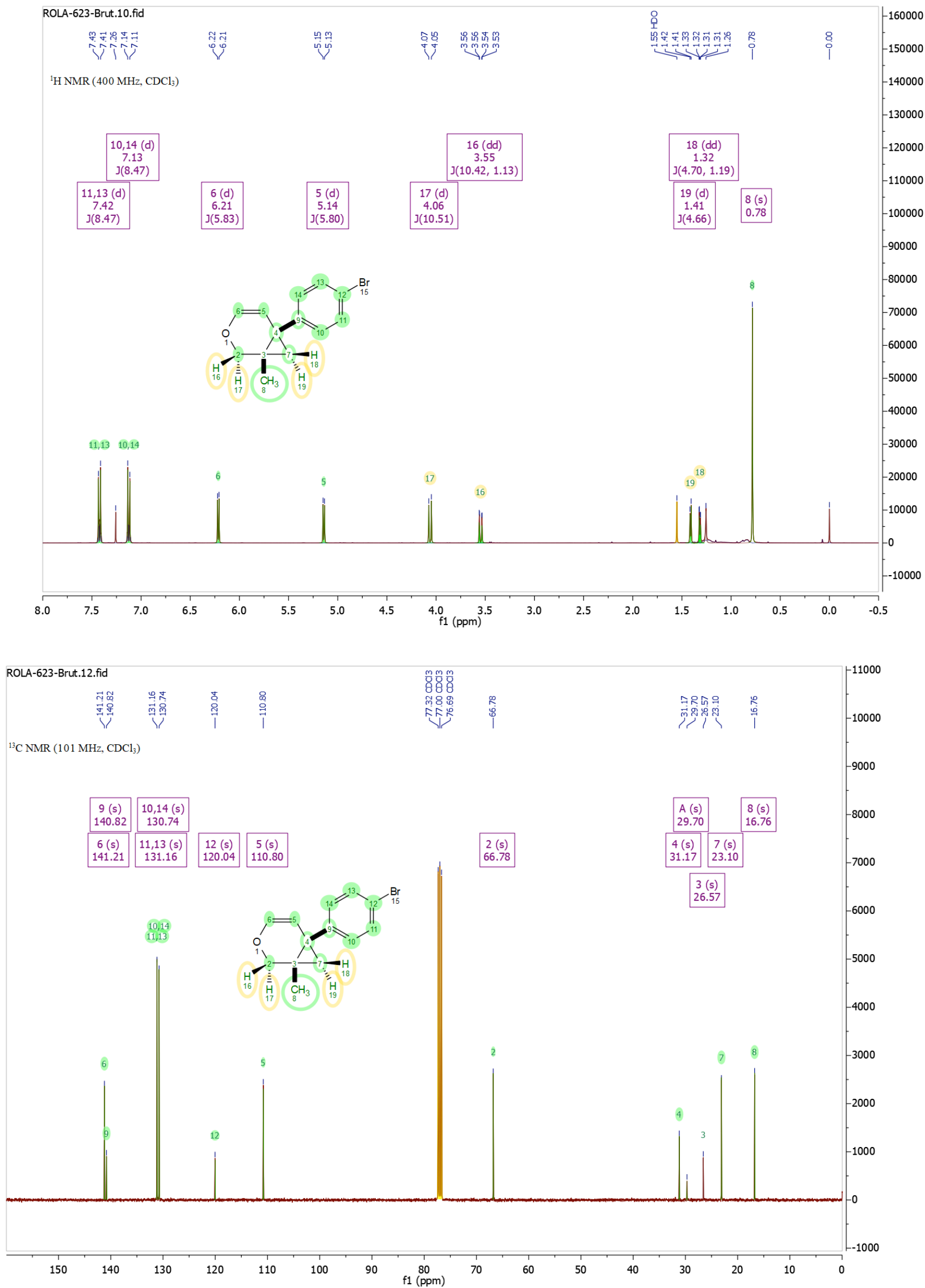
[2t]
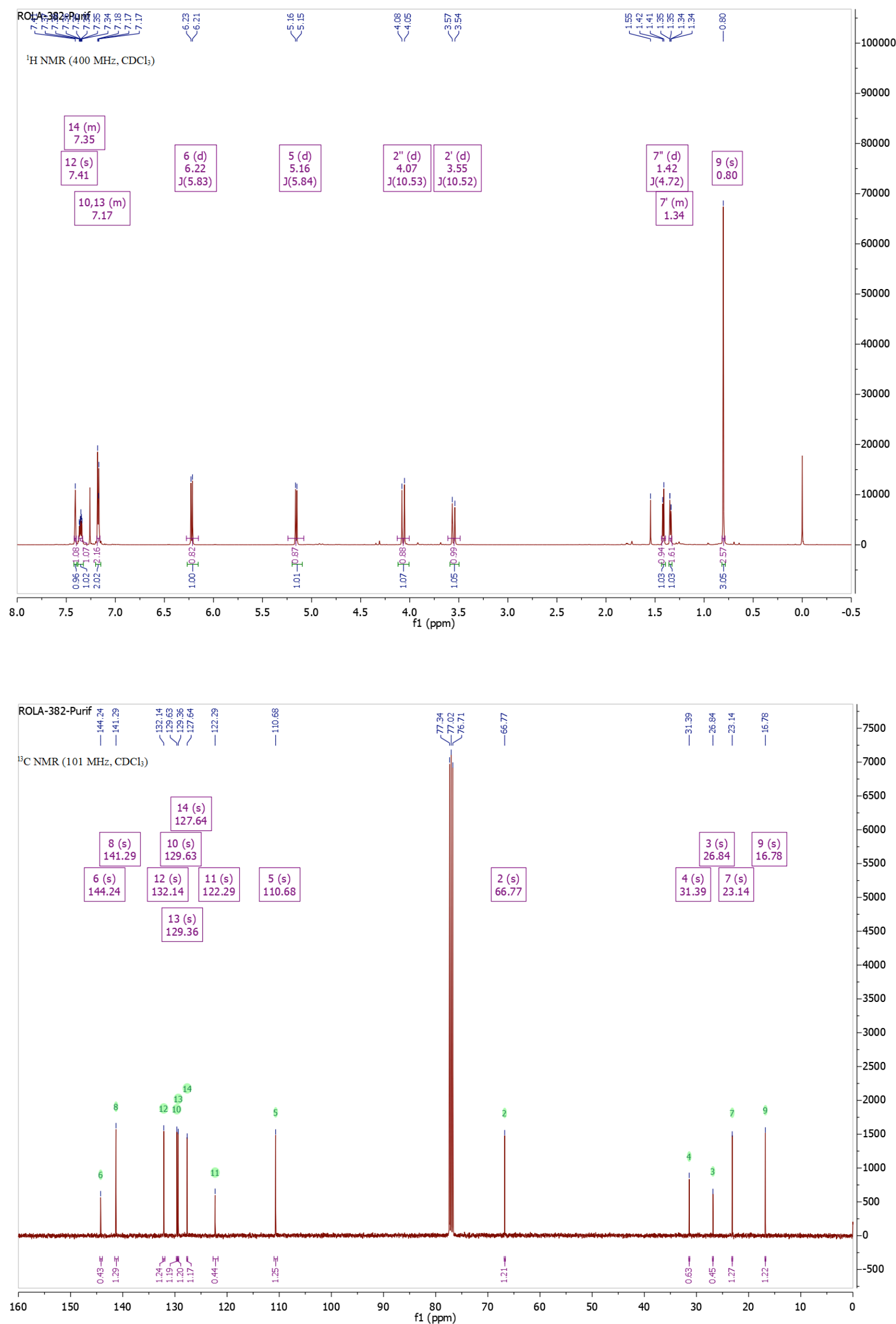
[2u]
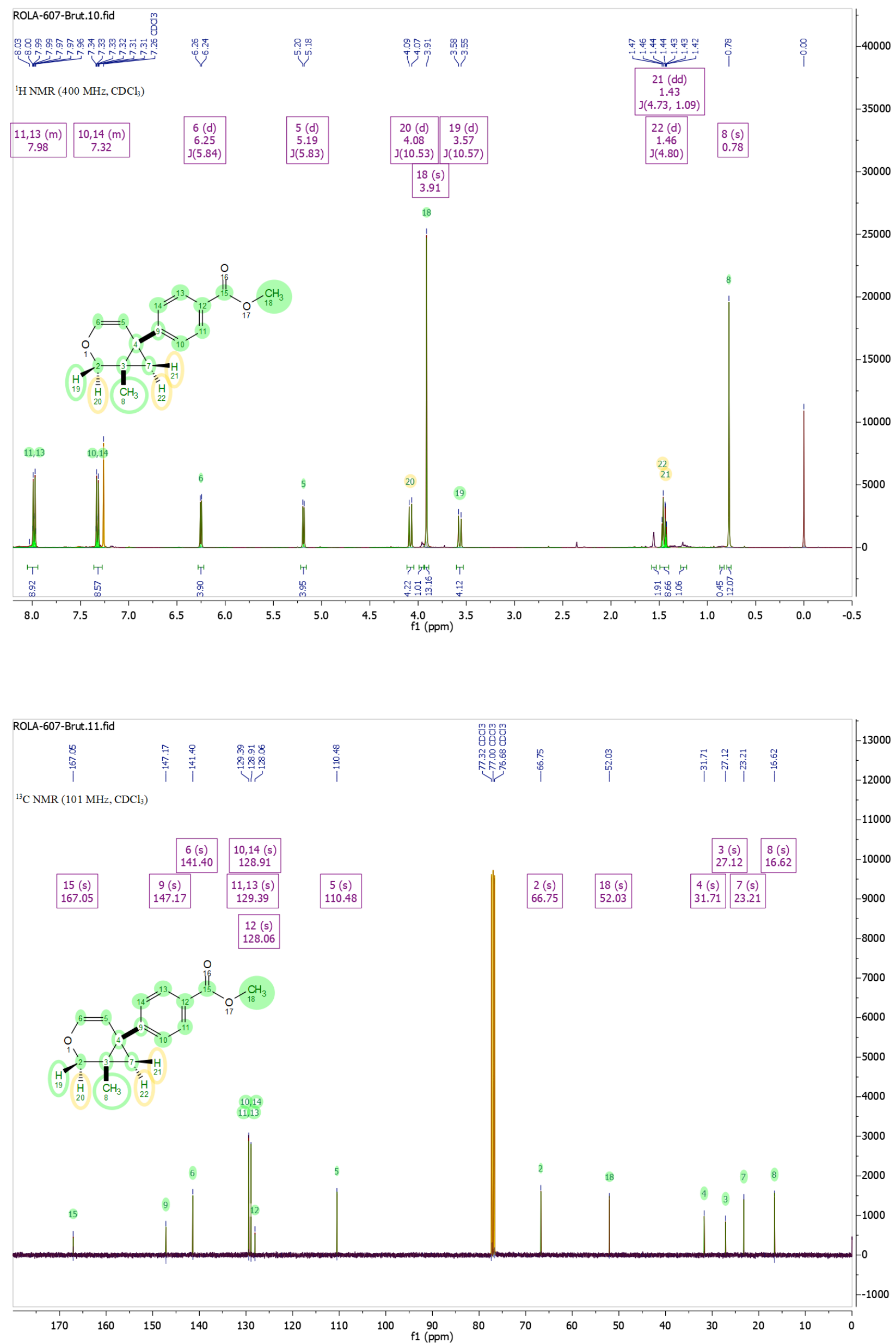
[v]
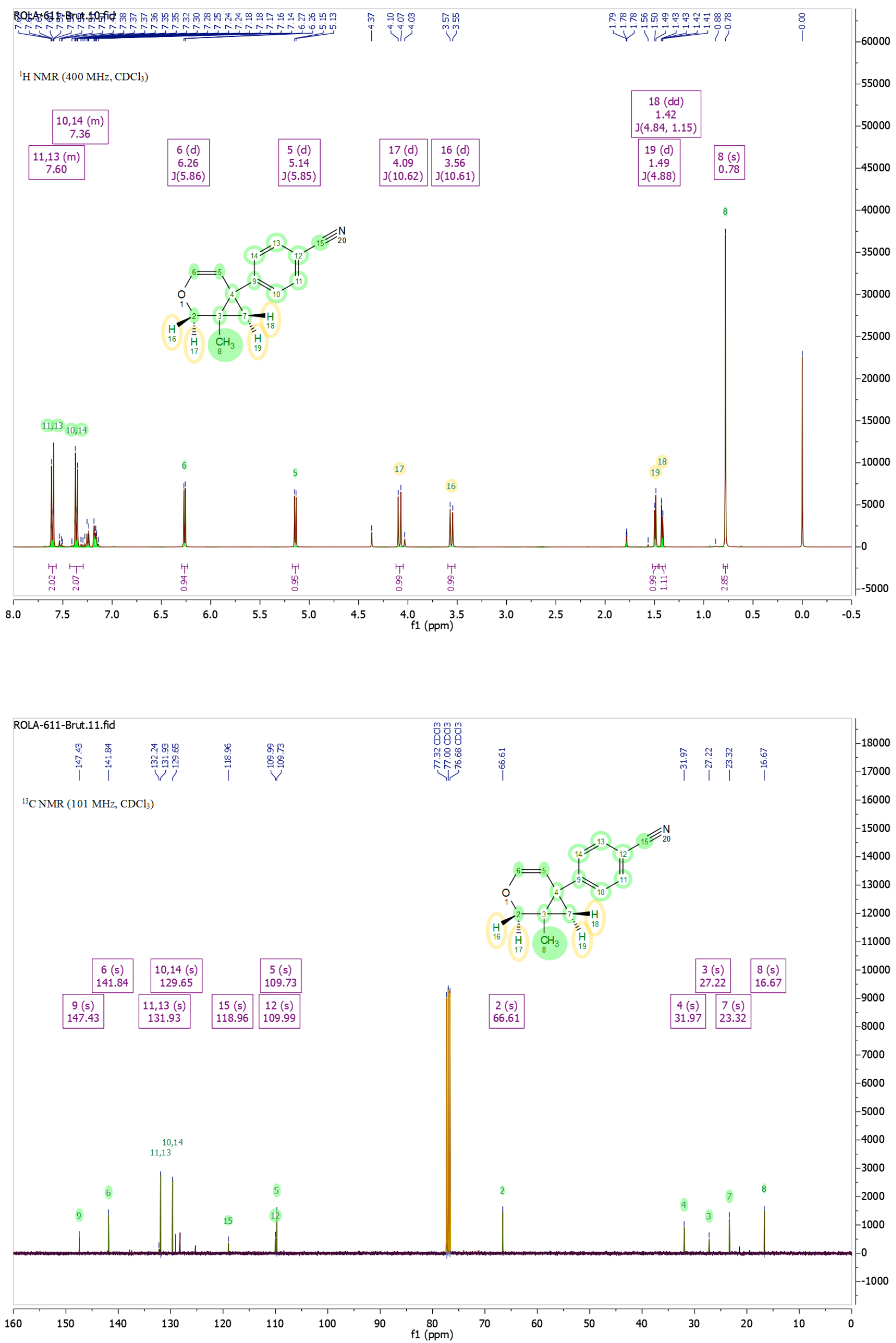
[4]
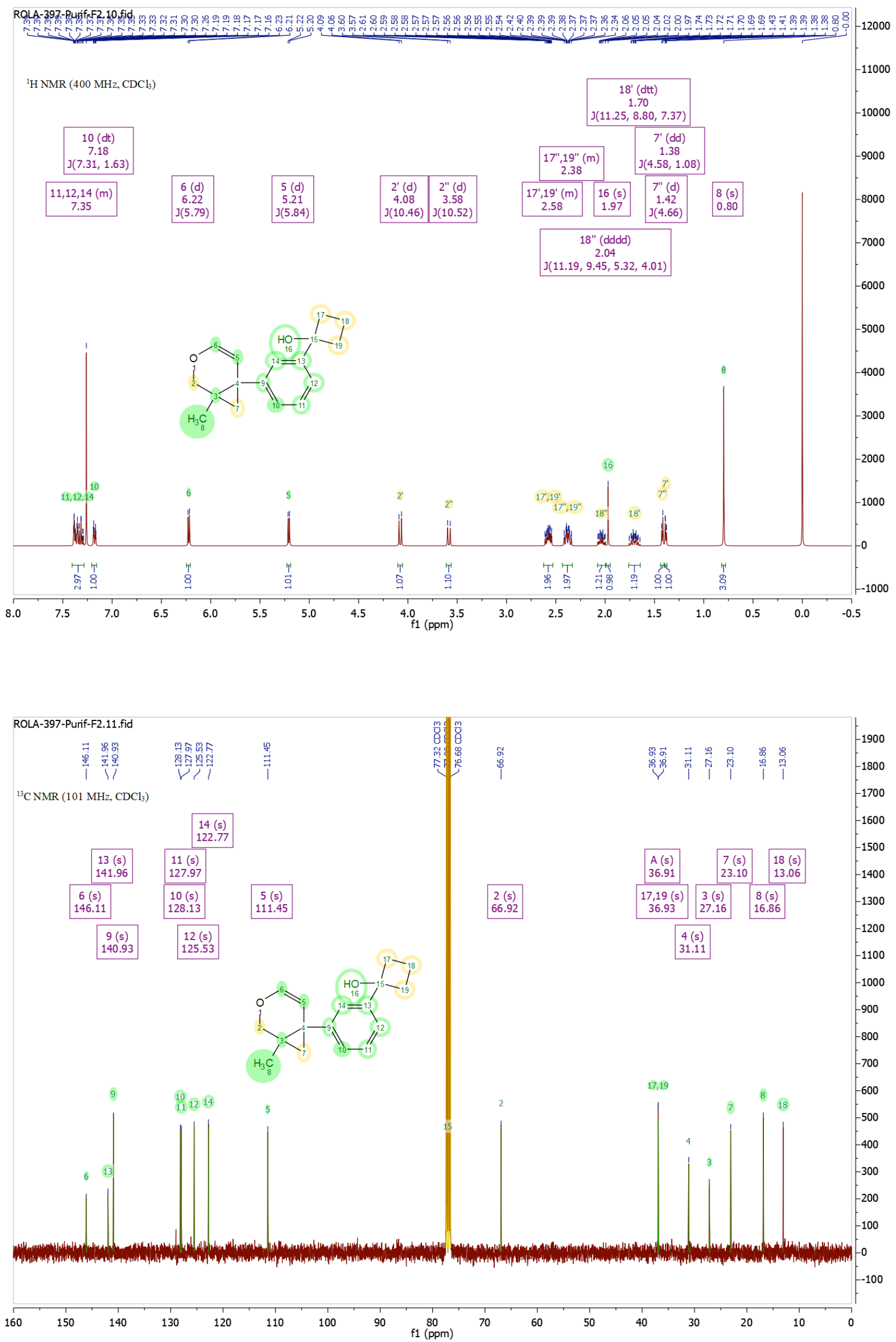
[5]
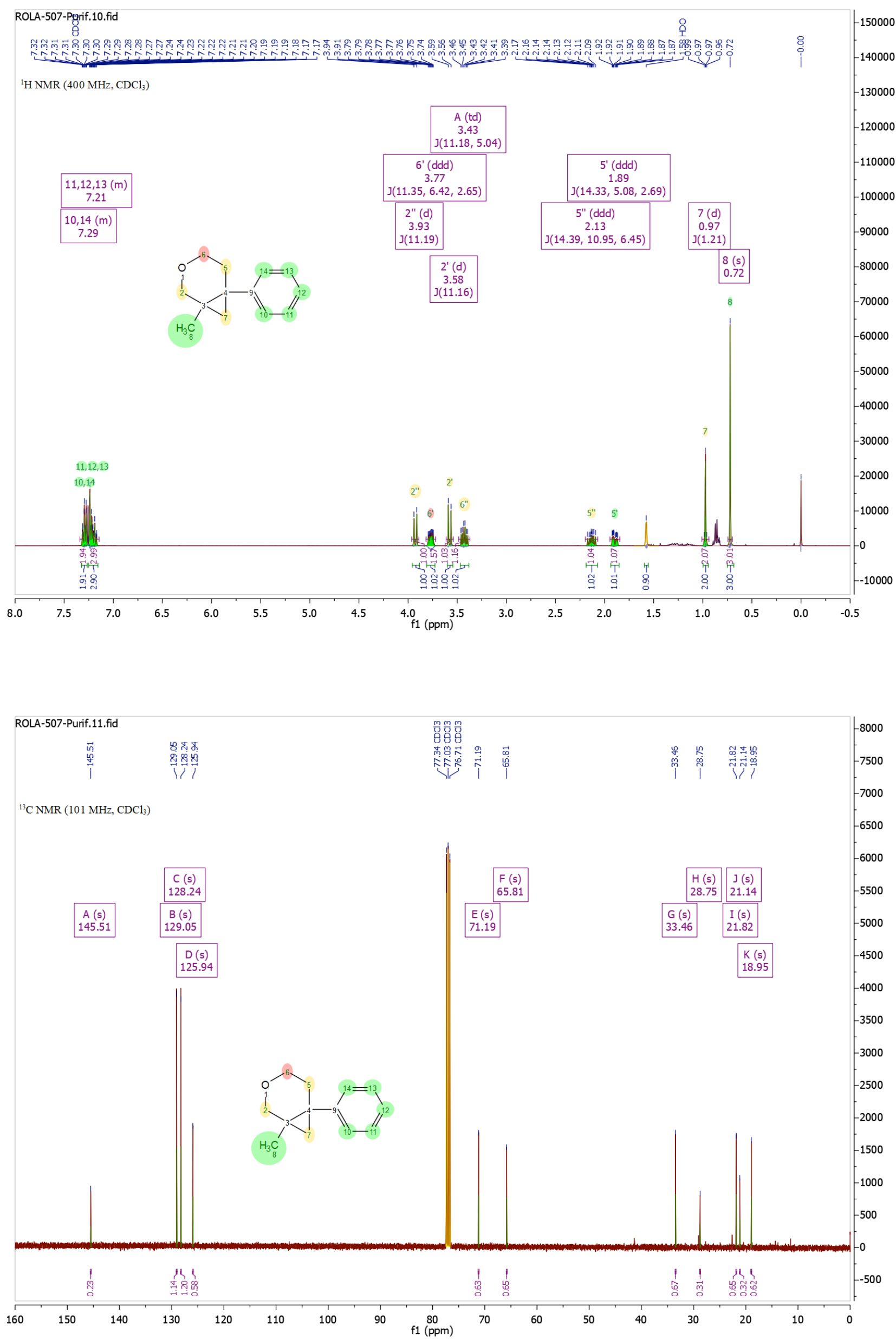


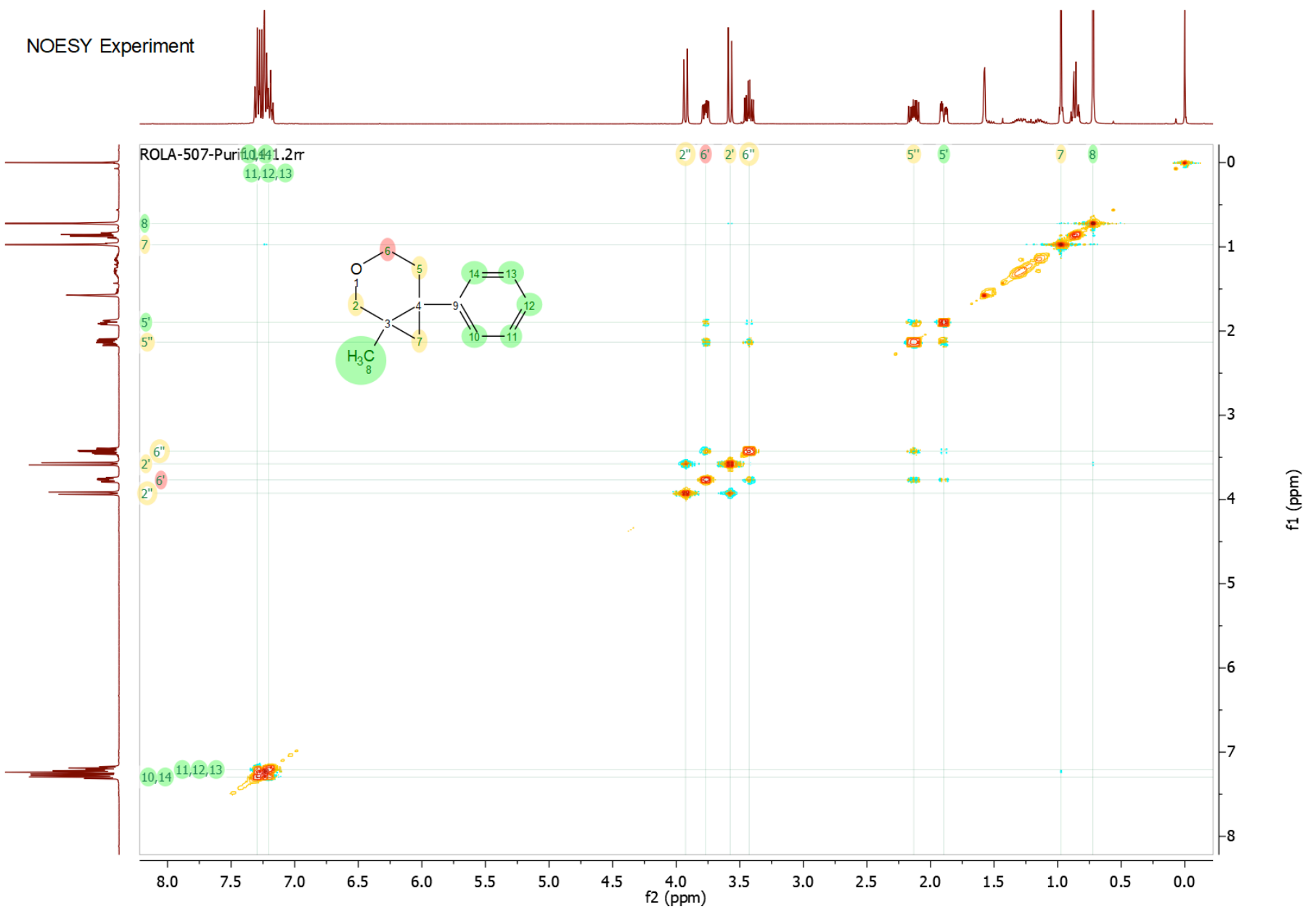


[6]
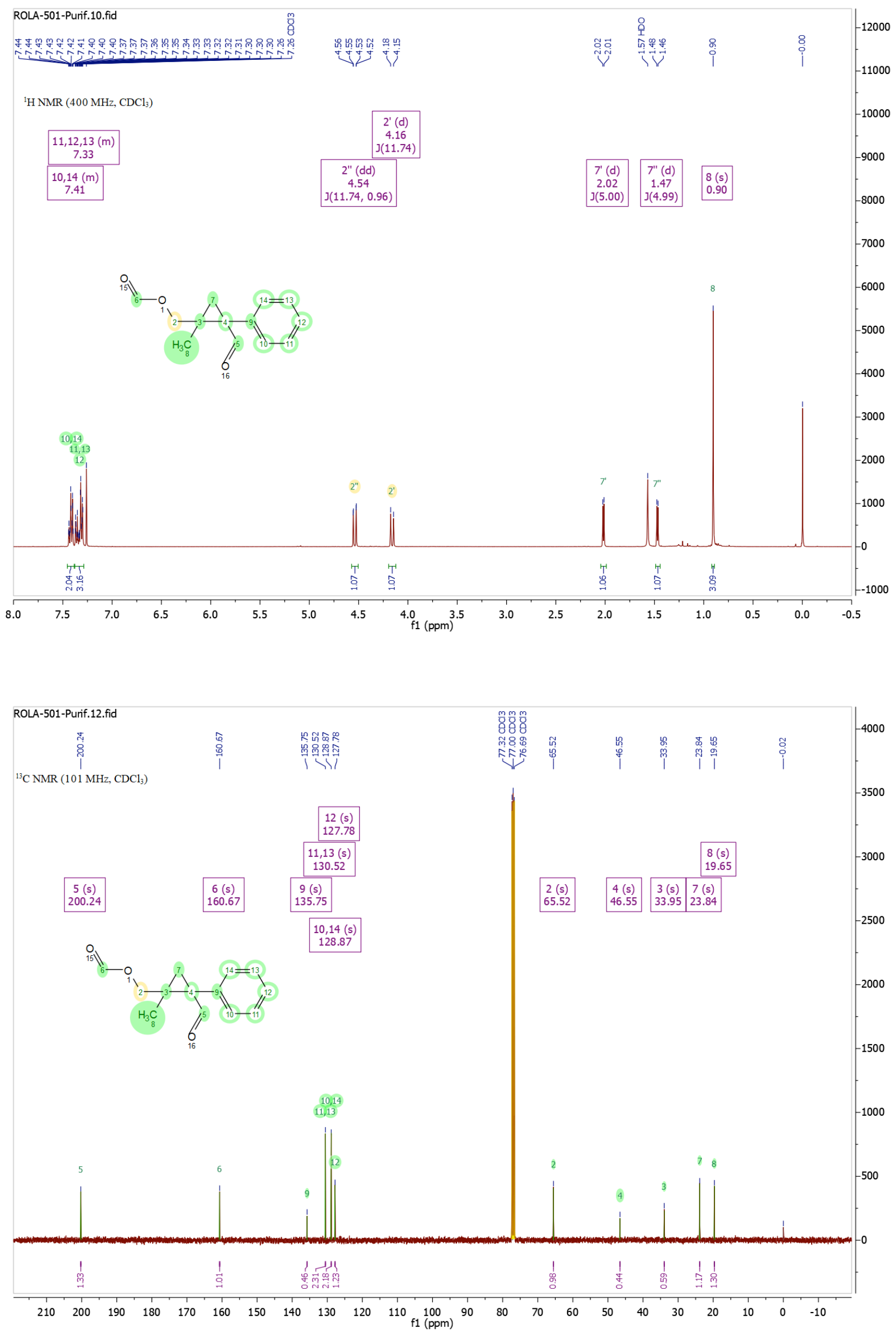

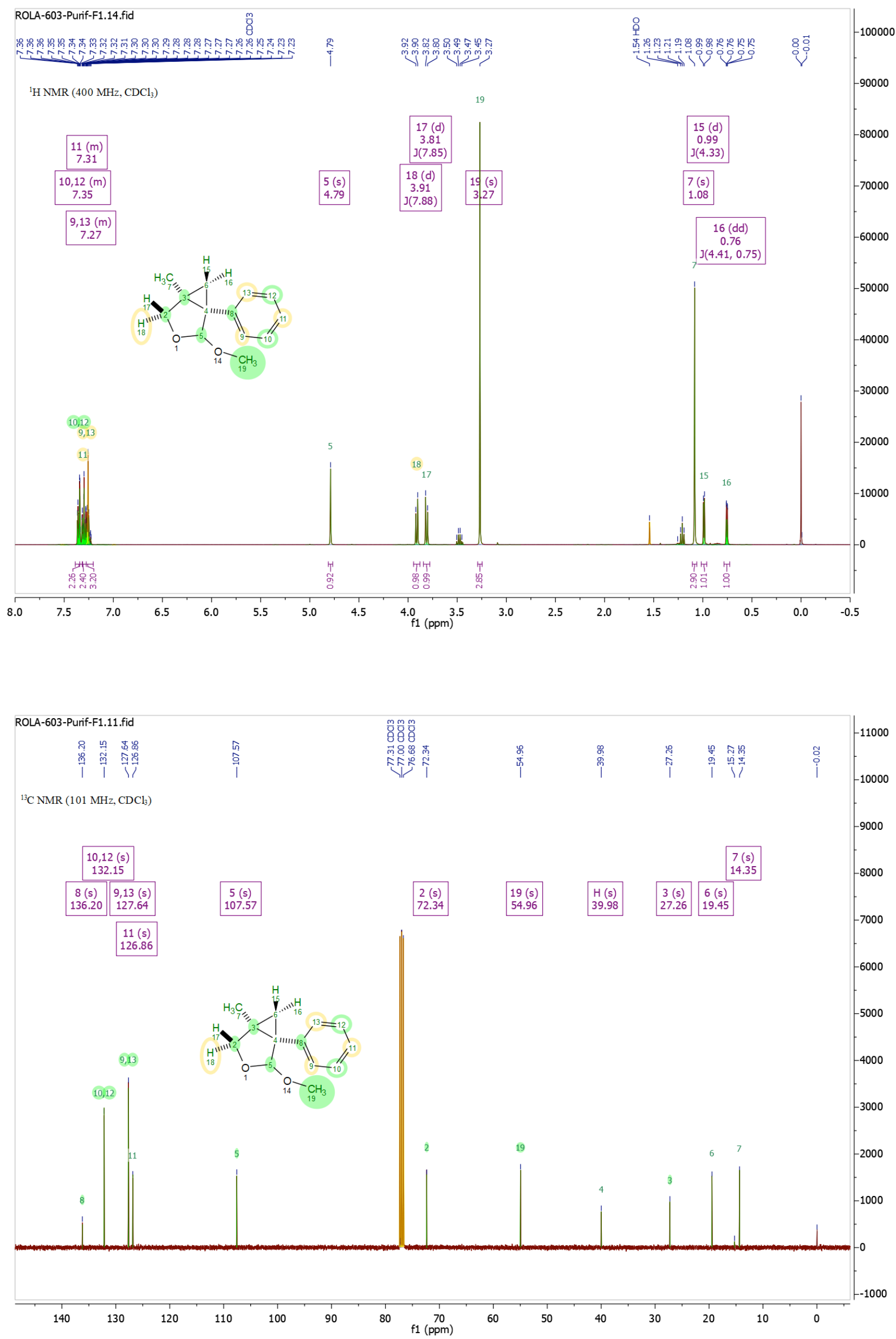
[8a]
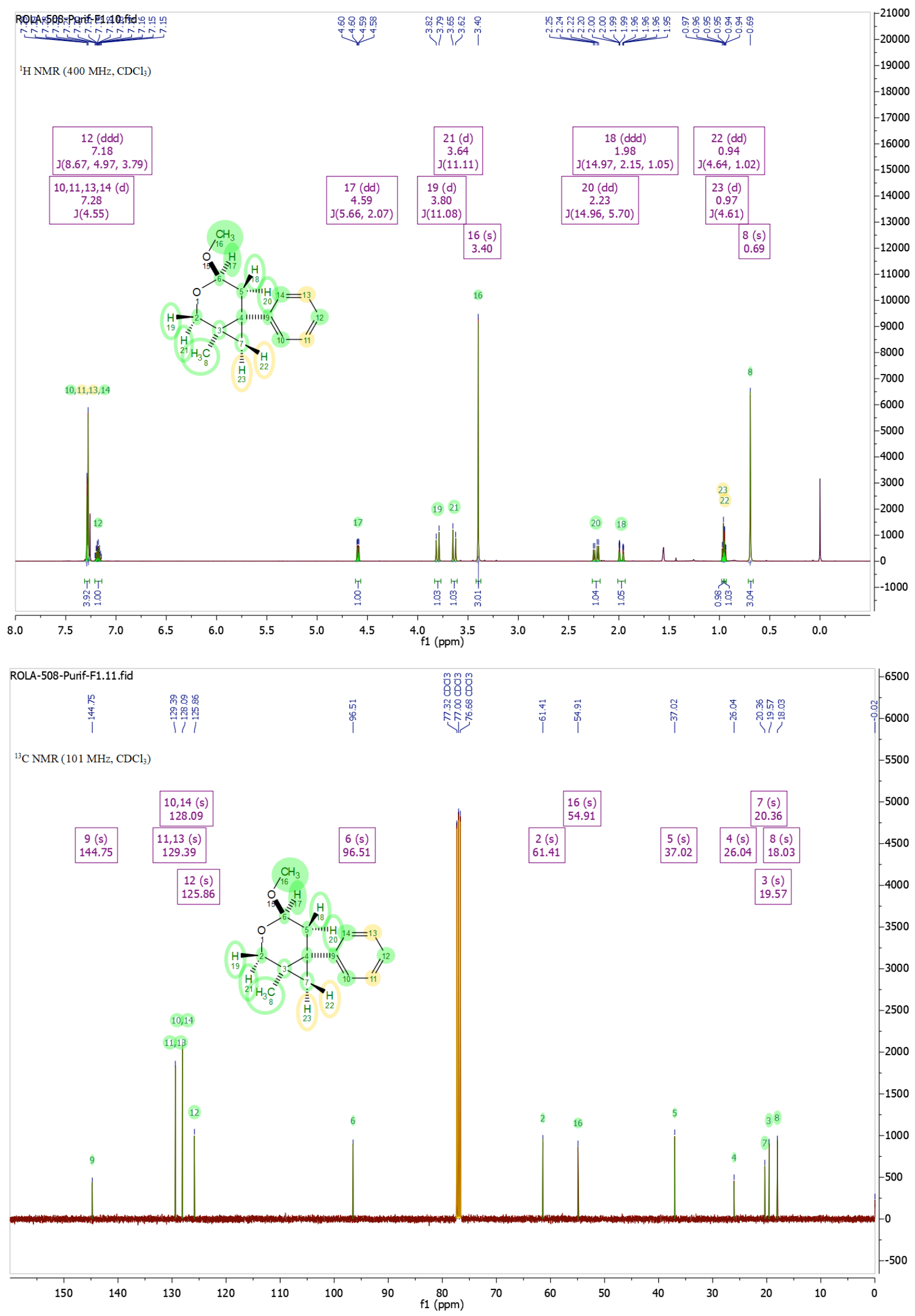
[8b]
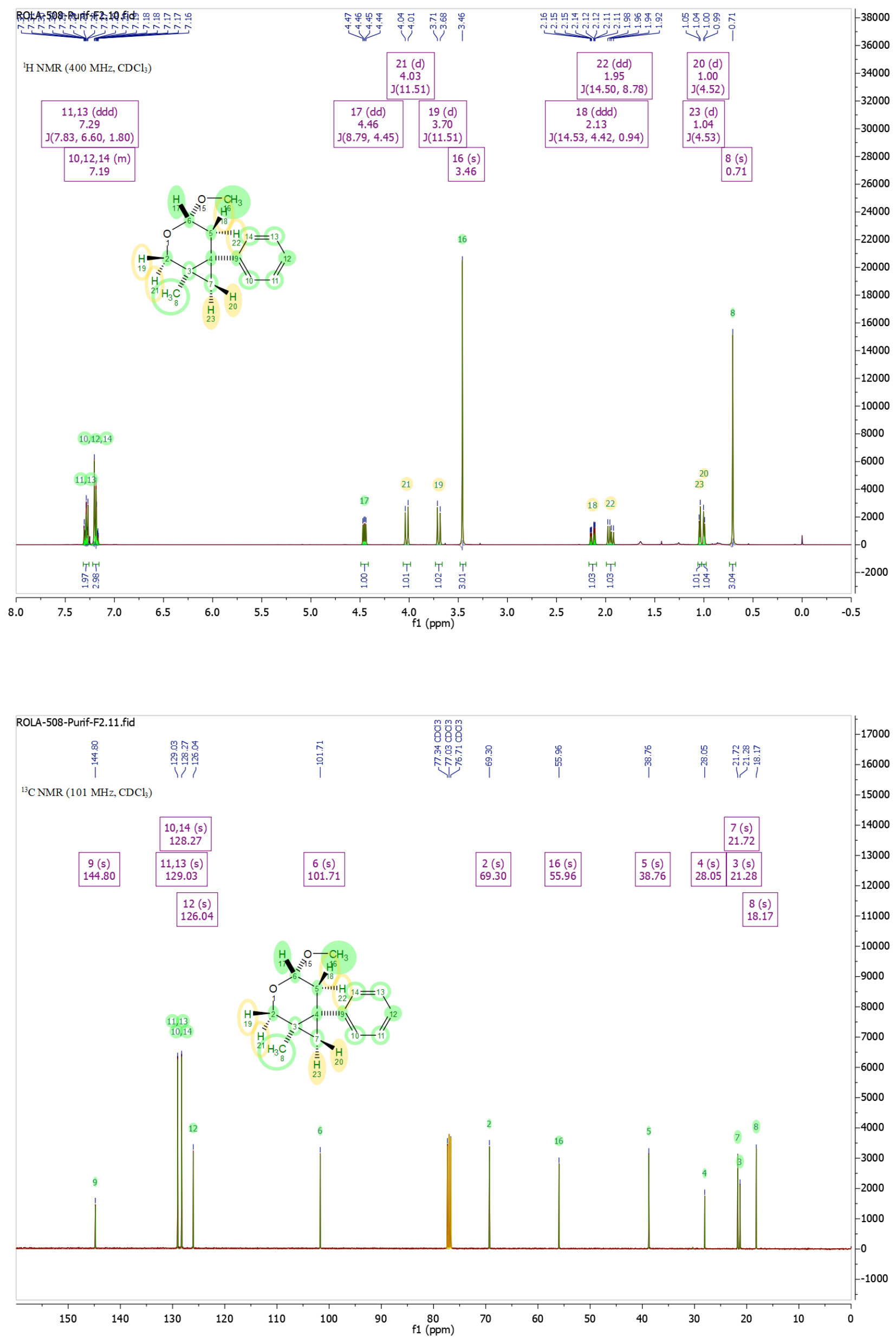